\title{
Les larves des Scarabaeoidea (Insecta, Coleoptera) en riziculture pluviale des régions de haute et moyenne altitudes du Centre de Madagascar
}

\author{
Richard RANDRIAMANANTSOA \\ Fofifa - URP Scrid, 110 Antsirabe (Madagascar) \\ r_randriamanantsoa@yahoo.fr \\ Henri-Pierre ABERLENC \\ Cirad Bios, UMR CBGP (INRA/IRD/Cirad/Montpellier SupAgro), \\ Campus international de Baillarguet, Ta A-55/L, \\ F-34398 Montpellier cedex 5 (France) \\ henri-pierre.aberlenc@cirad.fr \\ Olga Bakoly RALISOA \\ Département d'Entomologie, Faculté des Sciences, \\ Université d'Antananarivo, \\ Antananarivo 101 (Madagascar) \\ Alain RATNADASS \\ Cirad, UPR HortSys - ICRISAT BP 12404, Niamey (Niger) \\ a.ratnadass@cgiar.org \\ Bernard VERCAMBRE \\ Cirad, UPR Systèmes de Cultures annuels, \\ avenue Agropolis, F-34398 Montpellier cedex 5 (France) \\ bernard.vercambre@cirad.fr
}

MOTS CLÉS

Insecta,

Coleoptera,

Cetoniidae,

Melolonthidae,

Dynastidae,

Hopliidae,

Orphnidae, larve, nymphe,

structures ano-génitales

externes, raster.
Randriamanantsoa R., Aberlenc H.-P., Ralisoa O. B., Ratnadass A. \& Vercambre B. 2010. - Les larves des Scarabaeoidea (Insecta, Coleoptera) en riziculture pluviale des régions de haute et moyenne altitudes du Centre de Madagascar. Zoosystema 32 (1): 19-72.

\section{RÉSUMÉ}

Les larves de nombreuses espèces de Scarabaeoidea ("Vers blancs») sont présentes en riziculture pluviale des régions de haute et moyenne altitudes du Centre de Madagascar, parmi lesquelles certaines sont des ravageurs. Pouvoir les déterminer représente donc un important enjeu agronomique. La morphologie du raster permet de séparer les espèces. Vingt-six morpho-espèces larvaires ont été distinguées, parmi lesquelles 18 espèces, dont les larves et les nymphes étaient inconnues, ont été identifiées grâce aux imagos obtenus par élevage: Bricoptis, Celidota, Anochilia, Euryomia (Cetoniidae, quatre espèces), Heteronychus, Hexodon, Heteroconus, Paranodon (Dynastidae, sept espèces), Paramorphochelus (Hopliidae, une espèce), Encya, Enaria, Apicencya, Empecta, Hoplochelus (Melolonthidae, cinq espèces) et Triodontus (Orphnidae, une espèce). Les diagnoses originales 


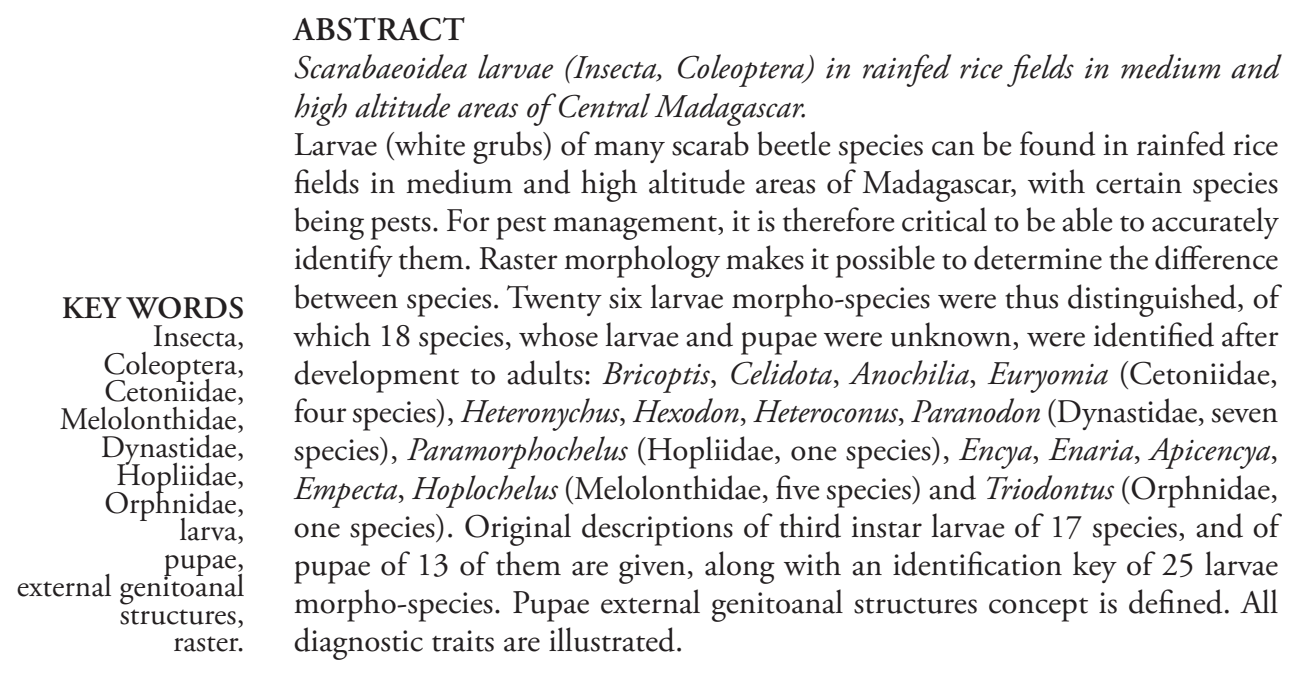

des larves de troisième stade de 17 espèces et des nymphes de 13 d'entre elles, ainsi qu'une clé d'identification de 25 larves sont proposées. Chez la nymphe, le concept de structures ano-génitales externes est défini. Tous les caractères diagnostiques sont illustrés.

\begin{abstract}
Scarabaeoidea larvae (Insecta, Coleoptera) in rainfed rice fields in medium and high altitude areas of Central Madagascar.

Larvae (white grubs) of many scarab beetle species can be found in rainfed rice fields in medium and high altitude areas of Madagascar, with certain species being pests. For pest management, it is therefore critical to be able to accurately identify them. Raster morphology makes it possible to determine the difference between species. Twenty six larvae morpho-species were thus distinguished, of which 18 species, whose larvae and pupae were unknown, were identified after development to adults: Bricoptis, Celidota, Anochilia, Euryomia (Cetoniidae, four species), Heteronychus, Hexodon, Heteroconus, Paranodon (Dynastidae, seven species), Paramorphochelus (Hopliidae, one species), Encya, Enaria, Apicencya, Empecta, Hoplochelus (Melolonthidae, five species) and Triodontus (Orphnidae, one species). Original descriptions of third instar larvae of 17 species, and of pupae of 13 of them are given, along with an identification key of 25 larvae morpho-species. Pupae external genitoanal structures concept is defined. All diagnostic traits are illustrated.
\end{abstract}

\section{INTRODUCTION}

Selon Ratcliffe \& Jameson (2004), la superfamille des Scarabaeoidea, dont les larves sont communément appelées "Vers blancs», compte dans le monde plus de 30000 espèces décrites, probablement même près de 35000 (Ratcliffe comm. pers.), dont quelques-unes sont des ravageurs importants des cultures.

Madagascar est riche d'une faune très diversifiée, avec une proportion élevée d'endémiques, y compris parmi les Scarabaeoidea. En riziculture pluviale des régions de haute et moyenne altitudes du Centre de Madagascar, de nombreux "Vers blancs» sont observés dans le sol, depuis la surface jusqu'à une profondeur d'une trentaine de centimètres (la grande majorité des larves se rencontrent entre 15 et $20 \mathrm{~cm})$.

Certains Scarabéides sont de redoutables ravageurs à Madagascar (Brenière \& Dubois 1965; Appert 1968). Quelques espèces des genres Heteronychus (Dynastidae) et Hoplochelus (Melolonthidae) sont des ennemis des cultures malgaches (Vercambre et al. 1990), mais les autres ne causent aucun dégât notable et favorisent même une bonne structure du sol (Ratnadass et al. 2006). L'identification des imagos est possible grâce aux volumes de la Faune de Madagascar (Paulian 1981, 1984; Dechambre 1986; Lacroix 1989, 1993, 1997, 1998), mais les larves, sauf rares exceptions, demeurent le plus souvent inconnues. Or l'identification des «Vers blancs» est un élément décisif pour définir de façon précoce les stratégies de contrôle à mettre en œuvre face aux espèces nuisibles.

Nous avons réalisé l'inventaire des larves de Scarabaeoidea présentes dans le système de riziculture pluviale des régions de haute et moyenne altitudes du Centre de Madagascar. Certains "Vers blancs» sont directement présents dans le sol des parcelles de Riz, d'autres sont apportés avec le fumier de Bovins. Grâce à des élevages en laboratoire, nous avons relié larves, nymphes et imagos, ce qui nous a permis de déterminer les espèces, d'établir une 


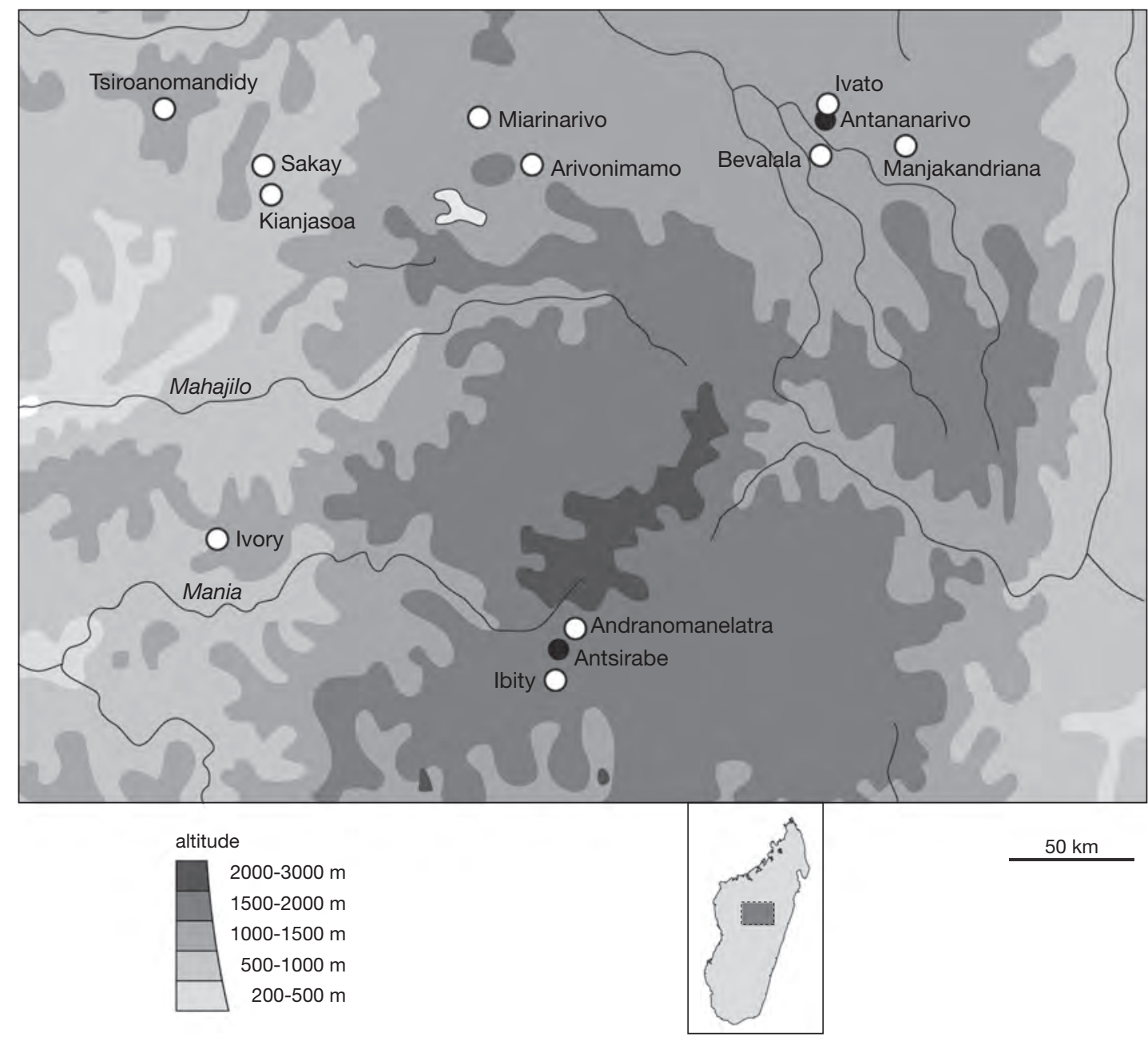

FIG. 1. - Carte de situation de la zone des prélèvements de larves de Scarabaeoidea à Madagascar.

clé d'identification des larves et d'en donner les diagnoses inédites.

\section{MATÉRIEL ET MÉTHODES}

\section{Collecte}

Les larves étudiées proviennent d'échantillonnages effectués de 2003 à 2005 dans les régions de haute et moyenne altitudes du Centre de Madagascar (entre $850 \mathrm{~m}$ et $1650 \mathrm{~m}$ ). Selon les localités, les précipitations annuelles s'échelonnent de 1200 à
$1500 \mathrm{~mm}$, avec des températures moyennes annuelles allant de 18 à $25^{\circ} \mathrm{C}$ (Jeanne et al. 2002).

Nos prélèvements sur le terrain (voir carte, Fig. 1) ont fait l'objet de deux protocoles distincts dans des stations représentatives de ce type particulier de rizières d'altitude, qui ne sont pas inondées, et de leur proche environnement. La nature des parcelles échantillonnées est précisée pour chaque espèce.

\section{Une prospection générale}

Les échantillonnages ont été réalisés en milieu paysan dans les régions du Bongolava, d'Itasy (axe 
TABLEAU 1. - Liste des localités prospectées.

\begin{tabular}{ll}
\hline Localités prospectées & Régions \\
\hline Sakay, Kianjasoa, & Bongolava \\
Arivonimamo Maharefo & Itasy \\
Ivato, Manjakandriana, Bevalala & Analamanga \\
Andranomanelatra, Ibity, & Vakinankaratra \\
Ambohimanabe, Ivory, Andranobe, & \\
Ankevobe, Ampandrotrarana, & \\
Antanikatsaka, Mangalaza, & \\
Ambohimiarivo & \\
\hline
\end{tabular}

Antananarivo-Tsiroanomandidy), d'Analamanga (Ivato, Bevalala, Manjakandriana) (Tableau 1). Dans chaque localité, des échantillons de sol ont été prélevés sur une surface de 2 ou $3 \mathrm{~m}^{2}$, à une profondeur de $20 \mathrm{~cm}$. Quand la densité larvaire était élevée, deux prélèvements de $1 \mathrm{~m}^{2}$ étaient faits séparément. Quand la densité larvaire était faible, le prélèvement était d'un seul tenant de $3 \mathrm{~m}^{2}$. Il arrivait même parfois qu'on ne trouve aucune larve dans un prélèvement de $3 \mathrm{~m}^{2}$. Des "Vers blancs" ont été également prélevés dans les parcs à Zébu, le fumier servant d'amendement (certaines larves ont été trouvées dans ces parcs et dans les parcelles).

\section{Un échantillonnage systématique}

Les échantillonnages ont été réalisés dans des stations expérimentales de la région du Vakinankaratra à Andranomanelatra (Centre) et à Ivory (Moyen Ouest). Les prélèvements étaient renouvelés régulièrement toutes les deux semaines, tout le long de l'année. Dans chaque station, trois parcelles adjacentes ont été étudiées. Pour chaque parcelle, 25 carrés de sol de $2 \times 2 \mathrm{~m}$ ont été prélevés, eux-mêmes découpés en 400 carrés de $10 \times 10 \mathrm{~cm}$, à trois profondeurs distinctes $(0-5 \mathrm{~cm} ; 5-10 \mathrm{~cm} ; 10-20 \mathrm{~cm})$, soit 1200 échantillons par carré.

La dynamique des populations fera l'objet de publications ultérieures.

Au champ, chaque morpho-espèce a été différenciée par l'examen du raster. Les "Vers blancs» collectés ont été soit mis en élevage en vue d'obtenir l'adulte, soit fixés au KAAD (Peterson 1960, 1962) et conservés ensuite à l'éthanol à $70 \%$.

Chaque morpho-espèce collectée étant morphologiquement caractérisée, un nom de code provisoire lui était attribué. Sauf exception, ce sont les larves du troisième stade, chez lesquelles les caractères sont les plus différenciés, qui ont été étudiées.

\section{ÉLEVAGE}

Les larves ont été élevées dans des boîtes rectangulaires en plastique $(6 \times 4,5 \times 4,5 \mathrm{~cm})$ munies d'un couvercle percé d'un trou de $5 \mathrm{~mm}$ de diamètre. Elles contenaient un substrat composé d'un mélange de débris végétaux secs et de terre préalablement chauffé à une température de $60{ }^{\circ} \mathrm{C}$ pendant 72 heures. Les boîtes d'élevage étaient placées dans une étuve maintenue à $25^{\circ} \mathrm{C}$.

\section{ILLUSTRATION}

Les photographies des plus gros spécimens ont été prises avec un appareil photo reflex numérique monté sur un statif de reproduction : soit un Nikon D70 muni d'un objectif macro AF Micro Nikkor $60 \mathrm{~mm} \mathrm{f} \mathrm{1:2,8} \mathrm{et} \mathrm{de} \mathrm{bagues} \mathrm{allonges,} \mathrm{soit} \mathrm{un} \mathrm{Canon}$ EOS 350D muni d'un objectif macro EF-S $60 \mathrm{~mm}$ f 1:2,8 USM. Les plus petits échantillons ont été photographiés sous une loupe binoculaire Leica MZ 16 équipée d'une caméra numérique Leica DFC 320. L'éclairage était obtenu soit par des fibres optiques, le double faisceau lumineux étant diffusé à travers un cylindre de papier calque, soit par une «boîte à lumière» spécialement conçue pour la photo des Insectes (Aberlenc 2006). Chaque image résulte de la fusion avec le logiciel Combine $Z 4$ de plusieurs photos (entre 2 et 25 ) réalisées en faisant varier la mise au point. Une partie des images a été faite avec une caméra JVC tri CCD KY-F75U montée sur une optique Leica Z16 APOA, à l'aide du logiciel Cartograph 6.3.1 de Microvision Instruments (Entovision).

\section{ABRÉVIATIONS}

CBGP Centre de Biologie pour la Gestion des Populations, Montpellier;

Cirad Centre de Coopération internationale en Recherche agronomique pour le Développement, Montpellier;

Fofifa Foibem-pirenena momba ny Fikarohana ampiharina amin'ny Fampandrosoana ny eny Ambanivohitra (Centre national de Recherches appliquées au Développement rural = Cenraderu), Madagascar;

MNHN Muséum national d'Histoire naturelle, Paris. 


\section{SYSTÉMATIQUE}

\section{INVENTAIRE DES ESPÈCES}

Vingt-six morpho-espèces de "Vers blancs " appartenant à six familles distinctes de Scarabaeoidea ont été distinguées grâce à l'examen du raster, dont cinq Cetoniidae (quatre espèces identifiées), neuf Dynastidae (six espèces et une sous-espèce identifiées), huit Melolonthidae (cinq espèces identifiées), un Hopliidae identifié, un Orphnidae identifié et deux Sericidae non identifiés. Sur ces 26 morpho-espèces, 20 ont pu être élevées jusqu’au stade imaginal et 18 ont pu être identifiées, soit 69,2\% (Tableau 2).

Si la littérature permet d'identifier les imagos, il nous a cependant semblé opportun de donner les photos des habitus et de certains détails morphologiques utiles pour leur détermination, afin de compléter la documentation disponible par une illustration inédite (Figs 23-25).

\section{DiAgNOSES DES LARVES}

Les échantillons sont conservés dans les collections du MNHN, du Cirad (CBGP) et du Fofifa. Nous utilisons la terminologie de Peterson $(1960,1962)$ et de Ritcher (1966).

\section{Famille CETONIIDAE Leach, 1815}

Bricoptis variolosa (Gory \& Percheron, 1833)

(Figs 2A; 3A; 6G; 10A; 14A-C; $16 \mathrm{E} ; 18 \mathrm{~A} ; 21 \mathrm{~A}-\mathrm{C} ; 23 \mathrm{~A}-\mathrm{C})$

\section{MATÉRIEL EXAMINÉ}

Les larves proviennent d'Andranomanelatra, de Sakay et d'Arivonimamo. Elles ont été trouvées dans une culture de Riz pluvial (Oryza sativa L.) et dans une jachère de Cynodon dactylon (L.) Pers., parcelles toutes deux situées en haut de pente, ainsi que dans $\mathrm{du}$ fumier de Bovins. Type de sol: alluvionnaire. Spécimens conservés dans les collections du Fofifa ( 5 larves), du Cirad-CBGP (4 larves) et du MNHN (1 larve).

\section{DESCRIPTION}

Couleur blanc ivoire (Fig. 2A).
TABLEAU 2. - Recensement (2003-2005) des larves de Scarabaeoidea présentes en riziculture pluviale des régions de haute et moyenne altitudes du Centre de Madagascar.

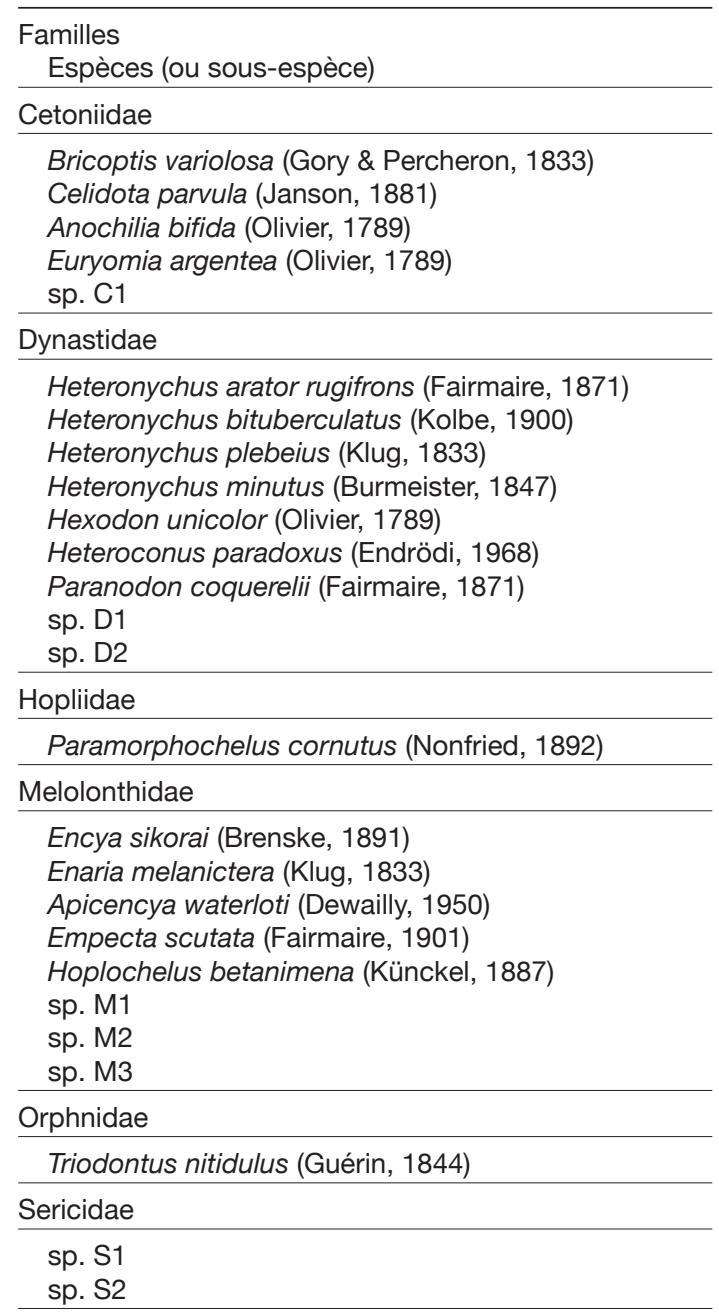

Tête

Couleur brun jaunâtre. Pas d'ocelle. 3 soies dorsoépicraniales; 2 soies épicraniales latéro-externes disposées symétriquement; 2 soies frontales médianes. 1 soie dans l'angle frontal. Clypéus transverse, caréné, avec 2 soies sur le postclypéus et 1 soie clypéale externe de chaque côté.

Antennes. Article I plus long que les II et III. Une aire sensorielle apico-latérale sur l'avant-dernier 
article, six aires sensorielles sur l'article apical: une petite aire à l'apex, entourée de trois plus grandes, plus une dorsale et une ventrale.

Labre. Transverse, faiblement trilobé, symétrique. Lobe médian avec 4 soies externes et 2 longues soies internes. Lobe latéral avec, d'avant en arrière, 5 soies antérieures (la plus latérale étant longue), 2 courtes soies suivies d'1 longue soie latérale et d'1 longue soie latéro-postérieure. 2 longues soies labro-internes. 4 soies postérieures (en 2 groupes de 2 soies).

Épipharynx (Fig. 6G). Haptomère formé d'une rangée transverse arquée de 12 courtes et fortes épines disposées en peigne, avec 3 fortes épines médianes en avant de l'aire pédiale, qui porte une vingtaine de soies fines et courtes peu denses. Chaetoparia avec 2 rangées concentriques de fortes soies convergeant sensiblement vers l'aire pédiale, les plus grandes étant à l'intérieur, avec des soies fines et courtes à la périphérie. Acanthoparia avec 5 à 7 soies de taille croissante d'arrière en avant. Concavité des clithra en arrondi peu profond et régulier. Une plaque sclérifiée circulaire.

Mandibules (Fig. 14A-C). Aire scissoriale de la mandibule gauche avec 4 dents, l'apicale allongée et étroite, les 2 suivantes très émoussées et arrondies, la dernière un peu plus saillante. Aire scissoriale de la mandibule droite avec 3 dents toutes très émoussées, les 2 dents antérieures plus grandes et plus saillantes. Sur la face ventrale des 2 mandibules, présence d'une aire stridulatoire de couleur plus claire composée de 38 à 42 bourrelets transverses. Mandibule gauche avec 3 soies baso-latérales et 2 soies dorso-externes. Mandibule droite avec 6 soies baso-latérales et 2 soies dorso-externes.

Maxilles (Fig. 16E). Galea et lacinia soudées. Lacinia à uncus avec 2 dents sclérifiées (une grande et une petite). Galea avec uncus sclérifié, large et émoussé. Sur la face dorsale, présence d'une aire stridulatoire composée de $5+1$ dents pointues coniques, faiblement espacées, inclinées vers l'avant.

Hypopharynx (Fig. 10A). Glosses : 2 rangées de 3 et 4 fortes soies inclinées vers l'intérieur à la base; une touffe de longues et fines soies sur le côté, au tiers apical.

Thorax

Pattes (Fig. 18A). Courtes. P1, P2 et P3 de structure analogue: coxa, trochanter, fémur tibia et tarse différenciés. Tarse allongé et pointu, plus pigmenté que les segments précédents, terminé par une courte et fine griffe sclérifiée.

\section{Abdomen}

Raster (Fig. 3A). Palidia formés de deux rangées longitudinales légèrement concaves de 12 à 19 épines. Septula glabre. Teges formés de soies courtes, peu denses. Fente anale transverse, faiblement arquée, proche de l'apex abdominal.

Celidota parvula (Janson, 1881)

(Figs 2B; 3B; 6H ; 10B; 13F-J ; 16F; 18B; 23D)

\section{MATÉRIEL EXAMINÉ}

Larves provenant d'Andranomanelatra, d'Ibity, de Kianjasoa, d'Arivonimamo et d'Ivato. L'espèce a été collectée à la fois dans une culture de Riz pluvial située en haut de pente, dans du fumier de Bovins, dans une jachère de Cynodon dactylon et dans un champ de Napier (Pennisetum purpureum Schum.) peu humide. Types de sol: alluvionnaire, de couleur noire, riche en matière organique. Spécimens conservés dans les collections du Cirad-CBGP (4 larves) et du MNHN (4 larves).

\section{DESCRIPTION}

Couleur blanc ivoire (Fig. 2B).

Tête

Couleur brun jaunâtre. Pas d'ocelle. 1 soie dorsoépicraniale et 2 soies épicraniales latéro-externes disposées symétriquement. Clypéus transverse avec 4 soies clypéales le long du bord postérieur et 2 soies clypéales externes de chaque côté.

Antennes. Article I plus long que les II et III, une aire sensorielle apico-latérale sur l'avant-dernier article, le dernier article antennaire avec cinq aires sensorielles: une petite aire apicale, 3 ventrales et 1 dorsale. 
Labre. Transverse, faiblement trilobé, subsymétrique. Lobe médian avec 6 fortes soies externes et 2 fortes soies internes. Lobe latéral avec, d'avant en arrière, 3 longues +4 courtes et épaisses soies antérieures externes et 3 longues soies + 1 longue antérieures internes; soies latérales et latéro-postérieures disposées en une rangée de 5 soies externes (la première étant longue) et une rangée de 4 soies internes. 2 longues et fortes soies labro-internes, avec en arrière de chacune et plus proches de l'axe médian une paire de courtes soies rapprochées. Une vingtaine de longues soies postérieures, formant une bande transverse allant d'un bord latéral à l'autre, disposées en 2 rangées irrégulières de soies.

Épipharynx (Fig. 6H). Haptomère avec une rangée transverse arquée de 12 courtes et fortes épines formant un peigne, avec postérieurement une rangée de 4 épines plus fortes. Aire pédiale avec de rares soies courtes, glabre au centre. Chaetoparia formés de rangées concentriques de soies longues et épaisses, nettement arquées vers l'avant, convergeant vers l'aire pédiale, avec de fines soies dispersées à la périphérie. Acanthoparia avec 7 à 9 longues soies de taille croissante vers l'avant. Clithra peu marqués mais présents.

Mandibules (Fig. 13F-J). Aire scissoriale de la mandibule gauche avec 4 dents développées, la première et la troisième plus aiguës (Fig. 13F, G). Aire scissoriale de la mandibule droite avec 3 dents développées, la première plus saillante et aiguë (Fig. 13H, I). Mandibule gauche avec 9 à 12 soies baso-latérales et deux soies dorso-externes. Mandibule droite avec 12 à 14 soies baso-latérales et 2 soies dorso-externes. Sur la face ventrale des 2 mandibules, présence d'une aire stridulatoire composée de 36 à 38 bourrelets parallèles (Fig. 13J).

Maxilles (Fig. 16F). Galea et lacinia soudées. Lacinia à uncus avec 2 dents sclérifiées développées (une grande et une petite), soudées à leur base. Galea avec un uncus sclérifié très pointu. Sur la face dorsale, présence d'une aire stridulatoire composée de $5+1$ petites dents pointues coniques très rapprochées, inclinées vers l'avant.
Hypopharynx (Fig. 10B). Glosses recouvertes de nombreuses longues soies inclinées vers l'intérieur, convergeant vers l'avant à l'apex et vers l'arrière à la base.

\section{Thorax}

Pattes (Fig. 18B). Courtes, de longueur croissante de P1 à P3, toutes de structure analogue: coxa, trochanter, fémur tibia et tarse différenciés. Tarse spatulé, sans griffe apicale, avec une touffe très dense de longues soies, de longueur croissante de P1 à P3.

\section{Abdomen}

Raster (Fig. 3B). Palidia formés de deux rangées longitudinales de 16 à 21 épines inclinées vers le septula et vers l'avant, convergeant à l'extrémité antérieure et divergeant à l'extrémité postérieure, avec une rupture de la courbe au tiers basal environ (formant ainsi le dessin d'un «fer de lance»). Septula glabre. Teges formés de courtes et de longues soies. Fente anale transverse, faiblement arquée, proche de l'apex abdominal.

Anochilia bifida (Olivier, 1789)

(Figs 2C; 3D; 6A; 7A; 10C; 12F;

$13 \mathrm{~A} ; 14 \mathrm{D}-\mathrm{G} ; 16 \mathrm{G} ; 18 \mathrm{C} ; 23 \mathrm{E}-\mathrm{G})$

\section{MatéRIEL EXAMINÉ}

Larves provenant d'Ibity. L'espèce a été collectée dans du fumier de Bovins (utilisé pour fertiliser les rizières). Spécimens conservés dans les collections du Fofifa (1 larve) et du Cirad-CBGP (2 larves).

\section{DESCRIPTION}

Couleur blanc ivoire (Fig. 2C).

\section{Tête (Fig. 6A)}

Couleur brunâtre. Pas d'ocelle. 1 soie dorso-épicraniale et 5 soies épicraniales latéro-externes. Pas de soie frontale. 1 soie dans l'angle frontal.

Antennes (Fig. 12F). Article I un peu moins long que les II et III pris ensemble. Une aire sensorielle apico-latérale sur l'avant-dernier article, 8 plages sensorielles sur l'article apical: une petite aire apicale, 4 ventrales et 3 dorsales. 
Labre (Fig. 13A). Transverse, trilobé, symétrique. Lobe médian avec 4 fortes soies externes et 4 soies internes. Lobe latéral avec, d'avant en arrière, 4 soies antérieures externes et 3 longues soies +1 très longue soie antérieures internes, et 1 longue soie latérale. 2 longues soies labro-internes. 10 à 12 courtes soies postérieures (en 2 groupes de 5 à 6 soies).

Épipharynx (Fig. 7A). Haptomère avec une rangée de 9 à 13 épines formant un peigne. Aire pédiale glabre. Chaetoparia dissymétriques, avec 2 rangées concentriques de soies convergeant vers l'aire pédiale du côté gauche, les plus internes plus fortes, et avec de nombreuses soies épineuses non alignées, devenant plus petites vers l'intérieur du côté droit, avec de fines soies du côté externe. Acanthoparia formés de 8 à 11 soies de taille croissante vers l'avant. Clithra bien marqués, la concavité étant légèrement anguleuse.

Mandibules (Fig. 14D-G). Aire scissoriale de la mandibule gauche avec 4 dents développées à bord arrondi. Aire scissoriale de la mandibule droite avec 3 dents, la première très développée et pointue. Mandibule gauche avec 3 longues +4 courtes soies baso-latérales et 2 soies dorso-externes. Mandibule droite avec 2 longues +6 courtes soies baso-latérales et 2 soies dorso-externes. Sur la face ventrale des 2 mandibules, présence d'une aire stridulatoire composée de 21 à 24 bourrelets transverses.

Maxilles (Fig. 16G). Galea et lacinia soudées. Lacinia à uncus avec 2 dents sclérifiées ( 1 grande et 1 petite), soudées à leur base. Galea avec uncus sclérifié. Sur la face dorsale, présence d'une aire stridulatoire composée de 6 à $8+1$ dents pointues, coniques, espacées, penchées vers l'avant.

Hypopharynx (Fig. 10C). Partie antérieure des glosses avec 2 rangées de 3 longues soies, les plus longues à l'apex, les plus courtes à la base. Une touffe de soies latérales à l'apex. En avant de l'oncylus, de courtes et fortes soies sont disposées en " $\mathrm{U}$ ». Oncylus transverse, fortement sclérifié, avec 2 lobes saillants.

\section{Thorax}

Pattes (Fig. 18C). Courtes, de longueur croissante de P1 à P3, toutes de structure analogue: coxa, trochanter, fémur tibia et tarse différenciés. Tarse court, fortement pigmenté, apex sclérifié avec une courte griffe.

\section{Abdomen}

Raster (Fig. 3D). Palidia formés de 2 rangées subparallèles, très légèrement incurvées et divergeant vers l'arrière, constituées de 11 à 14 épines inclinées vers le septula et vers l'arrière. Septula glabre. De chaque côté de la partie antérieure des palidia, 2 très longues soies, la plus interne en arrière, la plus externe en avant. Teges formés de soies courtes inclinées vers l'arrière. Fente anale transverse, faiblement arquée, proche de l'apex abdominal.

\section{Euryomia argentea (Olivier, 1789) \\ (Figs 2D; 3E; 7B; 10D; 14H-K; \\ $16 \mathrm{H} ; 18 \mathrm{D} ; 21 \mathrm{D}-\mathrm{F} ; 23 \mathrm{H})$}

\section{MATÉRIEL EXAMINÉ}

Larves provenant d'Ambohimanabe, à 6 km d'Antsirabe, dans une culture de Haricot (Phaseolus vulgaris L.) et de Patate douce (Ipomoea batatas L. (Lam.)). Les larves étaient localisées au niveau des racines des plantes. Elles avaient très probablement été apportées dans la parcelle avec le fumier de Bovins. Du Riz pluvial est cultivé en rotation sur cette parcelle. Spécimens conservés dans les collections du Fofifa (1 larve) et du MNHN (1 larve).

\section{DESCRIPTION}

Couleur blanc ivoire (Fig. 2D).

\section{Tête}

Couleur brunâtre. Pas d'ocelle. Épicrâne avec 2 soies dorso-épicraniales et 3 soies épicraniales latéroexternes. Front avec 2 soies frontales et 1 soie dans l'angle frontal. Clypéus transverse, caréné, avec 2 soies sur le postclypéus et 1 soie clypéale externe.

Antennes. Article I un peu plus long que les suivants (les longueurs respectives des articles sont: $\mathrm{I}=1 ; \mathrm{II}=0,66$; III $=0,58$ et $\mathrm{IV}=0,83)$. Une aire 
sensorielle apico-latérale sur l'avant-dernier article, cinq plages sensorielles sur l'article apical et une petite aire apicale.

Labre. Transverse, faiblement trilobé, symétrique. Lobe médian avec 5 soies externes et 2 soies internes. Lobe latéral avec, d'avant en arrière, 3 soies antérieures externes et 4 soies antérieures internes, la plus latérale longue, suivies de 2 soies latérales, la première longue. 2 longues soies labro-internes. 8 courtes soies postérieures (en 2 groupes transverses de 4 soies).

Épipharynx (Fig. 7B). Protophobe composé d'une rangée transverse faiblement arquée de fortes épines formant un peigne, plus courtes vers l'extérieur et plus longues dans la partie médiane. Dexiophobe composé d'une rangée de 9 longues épines inclinées vers le centre de l'aire pédiale. Laeophobe composé d'une rangée de 8 longues épines inclinées vers le centre de l'aire pédiale. Aire pédiale avec de courtes soies en arrière et 2 rangées de 3 et 2 fortes soies en avant. Chaetoparia avec des soies inclinées vers l'aire pédiale, de taille décroissante de l'intérieur vers l'extérieur. Acanthoparia formés de 7 à 9 courtes soies de taille croissante vers l'avant. Clithra anguleux, bien marqués.

Mandibules (Fig. 14H-K). Aire scissoriale de la mandibule gauche avec 4 dents bien développées, la première et la troisième pointues, la seconde et la quatrième arrondies. Aire scissoriale de la mandibule droite avec 3 dents à bord aplati, la première très pointue. Mandibules gauche et droite avec 5 soies baso-latérales, 2 soies dorso-externes et 1 soie dorso-molaire. Sur la face ventrale des 2 mandibules, présence d'une aire stridulatoire composée de 43 à 46 bourrelets transverses.

Maxilles (Fig. 16H). Galea et lacinia soudées. Lacinia avec 2 dents sclérifiées apicales, 1 très grande et 1 très petite, soudées à la base. Galea avec uncus sclérifié. Sur la face dorsale, présence d'une aire stridulatoire composée de $4+1$ dents pointues, coniques, espacées, inclinées vers l'avant.

Hypopharynx (Fig. 10D). Glosses: 2 rangées subparallèles de 4 soies convergeant vers l'apex; longues soies à l'apex, courtes soies à la base.

\section{Thorax}

Pattes (Fig. 18D). Courtes, toutes de structure analogue: coxa, trochanter, fémur tibia et tarse différenciés. Tarse allongé, pigmenté, apex avec une griffe sclérifiée aiguë.

\section{Abdomen}

Raster (Fig. 3E). Palidia formés de 2 rangées de 13 à 16 épines inclinées vers l'intérieur et vers l'arrière, formant une pointe en "ogive gothique» à l'extrémité antérieure. Septula glabre. Teges avec soies assez fortes de longueur moyenne, orientées vers l'arrière, souvent incurvées; de chaque côté de la partie antérieure des palidia, 2 très longues soies, la plus interne en arrière, la plus externe en avant. Fente anale transverse, faiblement arquée, proche de l'apex abdominal.

Famille Dynastidae MacLeay, 1819

\author{
Heteronychus arator rugifrons \\ (Fairmaire, 1871) \\ (Figs 2E; 3F; 7C; 10E; 14L-O; \\ 16I, J; 19A; 21G-I; 25A-C)
}

\section{MATÉRIEL EXAMINÉ}

Larves provenant d'Andranomanelatra, d'Ivory, d'Ibity, d'Andranobe (7 km d'Antsirabe), de Sakay, de Kianjasoa, d'Arivonimamo, d'Ivato et de Manjakandriana. Ce taxon a été collecté dans une jachère de Cynodon dactylon avec du Trèfle (Trifolium semipilosum Fresen.) à Andranomanelatra, dans une culture de Napier (Pennisetum purpureum) à Ivato, dans une culture de Riz pluvial (Oryza sativa) et de Maïs (Zea mays L.) à Andranomanelatra, Ivory, Ibity, Andranobe, Sakay, Kianjasoa, Arivonimamo et Manjakandriana. Il se rencontre fréquemment en haut de pente. Type de sol: alluvionnaire, ferrallitique, de couleur noire, riche en matière organique. Spécimens conservés dans les collections du Fofifa (14 larves), du Cirad-CBGP (20 larves) et du MNHN (5 larves).

\section{DESCRIPTION}

Couleur blanche (Fig. 2E). 
Tête

Couleur brun clair. Épicrâne avec 2 soies dorsoépicraniales et 5 soies latéro-externes. Front avec 4 soies frontales antérieures, 2 soies frontales postérieures et 2 soies dans l'angle frontal. Clypéus transverse, avec 1 soie clypéale externe et 1 soie clypéale antérieure médiane.

Antennes. Article II un peu plus long que les autres (les longueurs respectives des articles sont: $I=1$; $\mathrm{II}=1,3$; III = IV = 1,1). Une aire sensorielle apicolatérale sur l'avant-dernier article. Dernier article antennaire avec 3 plages sensorielles : 2 ventrales et 1 grande plage dorsale.

Labre. Transverse, asymétrique. Zone médiane $\mathrm{du}$ bord antérieur à peine saillante, légèrement différenciée du côté droit, avec 4 soies externes et 2 soies internes. De part et d'autre, 5 soies latéro-antérieures externes et 2 longues soies latéro-antérieures internes, puis 1 longue soie latérale. 2 longues soies labro-internes en avant du milieu. 4 soies postérieures (en 2 groupes de 2 soies).

Épipharynx (Fig. 7C). Haptomère entier avec une forte pointe sclérifiée relevée arrondie. Aire pédiale glabre. Chaetoparia dissymétriques, à rangs concentriques de soies épaisses convergeant vers l'aire pédiale, plus longues et plus denses du côté gauche, plus courtes et plus dispersées du côté droit. Acanthoparia avec 11 à 13 courtes soies lamelliformes s'incurvant graduellement vers l'avant. Plegmatium et proplegmatium absents.

Mandibules (Fig. 14L-O). Aire scissoriale de la mandibule gauche avec une forte dent apicale et la seconde dent scissoriale plus ou moins émoussée. Aire scissoriale de la mandibule droite avec 2 dents obtuses presque également développées. Mandibule gauche avec 7 à 8 soies baso-latérales, 2 soies dorsoexternes écartées et 5 à 6 soies dorso-molaires. Mandibule droite avec 6 soies baso-latérales, 2 soies dorso-externes écartées et 5 à 6 soies dorso-molaires. Sur la face ventrale des 2 mandibules, présence d'une aire stridulatoire composée de 21 à 23 bourrelets parallèles transverses.
Maxilles (Fig. 16I, J). Galea et lacinia soudées. Lacinia à uncus tridenté. Galea avec un uncus sclérifié. Sur la face dorsale, aire stridulatoire composée de 7 à $10+1$ dents aux bords arrondis émoussés (Fig. 16J).

Hypopharynx (Fig. 10E). Glosses avec deux touffes de longues soies apicales et de courtes et fortes soies à la base, en avant des oncyli, ces derniers transverses, avec une dent sclérifiée postérieure subtriangulaire et une forte dent antérieure, sclérifiée et dressée.

\section{Thorax}

Pattes (Fig. 19A). Longues, de longueur croissante de P1 à P3, toutes de structure analogue: coxa, trochanter, fémur, tibiotarse, griffe longue, aiguë, incurvée et sclérifiée à l'apex.

\section{Abdomen}

Raster (Fig. 3F). Fente anale transverse, éloignée de l'apex abdominal. Du côté dorsal de la fente anale, 2 plages de courtes et fortes soies inclinées vers le centre, séparées par un septum glabre bien délimité. Du côté ventral de la fente anale, et séparée de celle-ci par une étroite bande glabre, une rangée transverse de longues soies dirigées vers le milieu et absentes en zone centrale; teges rassemblant un groupe de courtes et fortes soies épineuses.

Heteronychus bituberculatus (Kolbe, 1900)

(Figs 2F; 3G; 7D; 10F; 14P-S ;

$16 \mathrm{~K}, \mathrm{~L} ; 19 \mathrm{~B} ; 25 \mathrm{D}-\mathrm{G})$

\section{MATÉRIEL EXAMINÉ}

Larves provenant d'Andranomanelatra, de Sakay, de Kianjasoa, de Bevalala et d'Ivato. L'espèce a été collectée respectivement dans une jachère de Cynodon dactylon, dans des cultures de Riz pluvial (Oryza sativa), de Maïs (Zea mays), de Napier (Pennisetumpurpureum), de Brachiaria ruziziensis R.Germ. \& C.M.Evrard, de Patate douce (Ipomoea batatas), près de Roseaux et de Graminées diverses. Elle se rencontre généralement dans les lieux humides en bas de pente. Types de sol: alluvionnaire, argileux, ferrallitique, volcanique. Spécimens conservés dans les collections du Fofifa 
(6 larves), du Cirad-CBGP (2 larves) et du MNHN (1 larve).

\section{DESCRIPTION}

Couleur blanche (Fig. 2F).

Tête

Couleur jaunâtre. Épicrâne avec 2 soies dorsoépicraniales et 3 soies latéro-externes de chaque côté. Front avec 2 soies frontales postérieures. 2 soies, 1 grande et 1 petite, dans l'angle frontal. Clypéus transverse, avec 1 soie latérale externe et 2 soies antérieures.

Antennes. Article II un peu plus long que les autres (les longueurs respectives des articles sont: $\mathrm{I}=1 ; \mathrm{II}=1,5 ; \mathrm{III}=\mathrm{IV}=1,4)$. Une aire sensorielle apico-latérale sur l'avant-dernier article. Dernier article antennaire avec 4 plages sensorielles: 1 petite plage apicale ronde, 2 ventrales et 1 grande plage dorsale.

Labre. Transverse, asymétrique. Zone médiane du bord antérieur saillante, formant presque un lobe médian différencié à droite, avec 4 soies antérieures et 2 soies postérieures. De part et d'autre, 2 soies latéro-antérieures externes et 2 soies latéro-antérieures internes, puis 1 longue soie latérale. 2 longues soies labro-internes en avant du milieu. 4 soies postérieures (en 2 groupes transverses de 2 soies).

Épipharynx (Fig. 7D). Haptomère transverse, sclérifié, pointu. Aire pédiale glabre. Chaetoparia dissymétriques, avec de 1 à 5 soies courtes et épaisses convergeant vers l'aire pédiale, qui est glabre. Acanthoparia avec 8 à 10 courtes soies lamelliformes incurvées vers l'avant. Plegmatium et proplegmatium absents.

Mandibules (Fig. 14P-S). Aire scissoriale de la mandibule gauche avec une forte dent apicale émoussée et un bord interne irrégulier, une dent pointue très obtuse en zone médiane. Aire scissoriale de la mandibule droite avec une forte dent apicale émoussée, le bord interne inerme. Mandibules gauche et droite avec chacune 6 soies baso-latérales et 1 soie dorso-externe. Sur la face ventrale des 2 mandibules, présence d'une aire stridulatoire composée de 22 à 23 bourrelets parallèles transverses.

Maxilles (Fig. 16K, L). Galea et lacinia soudées. Lacinia à uncus avec 2 dents. Galea avec uncus sclérifié. Sur la face dorsale, aire stridulatoire à 5 grosses dents irrégulièrement écartées à apex très émoussé (Fig. 16L).

Hypopharynx (Fig. 10F). Glosses recouvertes de courtes et épaisses soies moins denses au milieu et de longues soies vers l'apex et sur les côtés. Oncyli transverses, avec une dent sclérifiée postérieure subtriangulaire et une forte dent antérieure, sclérifiée et dressée.

\section{Thorax}

Pattes (Fig. 19B). Longues, de longueur croissante de P1 à P3, toutes de structure analogue: coxa, trochanter, fémur très allongé, tibiotarse, griffe forte, longue, aiguë, incurvée et sclérifiée à l’apex.

\section{Abdomen}

Raster (Fig. 3G). Fente anale transverse, éloignée de l'apex abdominal. Côté dorsal de la fente anale présentant de très courtes et fortes soies inclinées vers l'apex constituant une plage continue transverse. Les soies du côté ventral de la fente anale sont plus longues, plus denses et plus fortes, de section cylindrique. Teges avec de fortes soies aplaties à l'apex incurvé. Barbula avec de longues et fines soies dispersées.

Heteronychus plebeius (Klug, 1833)

(Figs 2G; 3H; 10G; 11A, E-H ; 12G; 13B; 16M; 19C; 21J-Q; 25H-J)

\section{MATÉRIEL EXAMINÉ}

Larves provenant de Sakay, de Kianjasoa, de Maharefo et d'Ivato. L'espèce a été collectée dans des cultures de Riz pluvial (Oryza sativa), de Napier (Pennisetum purpureum), situées en haut de pente. Type de sol: alluvionnaire, de couleur noire, riche en matière organique. Spécimens conservés dans les collections du Fofifa (4 larves), du Cirad-CBGP (3 larves) et du MNHN (2 larves). 
DESCRIPTION

Couleur blanc ivoire (Fig. 2G).

Tête

Couleur jaunâtre sombre. Épicrâne avec 2 soies dorso-épicraniales et 4 soies latéro-externes disposées symétriquement. Front avec 2 soies frontales antérieures et 2 soies frontales postérieures. 1 soie dans l'angle frontal. Clypéus transverse avec 1 soie latérale externe et 2 soies antérieures.

Antennes (Fig. 12G). Article II un peu plus long que les autres (les longueurs respectives des articles sont: $\mathrm{I}=1 ; \mathrm{II}=1,4 ; \mathrm{III}=1,16 ; \mathrm{IV}=1,08)$. Une aire sensorielle apico-latérale sur l'avant-dernier article. Dernier article antennaire avec 3 plages sensorielles: 2 ventrales et une grande plage dorsale.

Labre (Fig. 13B). Transverse, asymétrique. Zone médiane du bord antérieur peu saillante, formant à peine un lobe médian, légèrement différencié du côté droit, avec 4 soies antérieures externes et 2 soies antérieures internes. De part et d'autre, 3 soies latéro-antérieures externes et 2 soies latéro-antérieures internes, puis une longue soie latérale. 2 longues soies labro-internes en avant du milieu. 4 soies postérieures (en 2 groupes transverses de 2 soies).

Épipharynx (Fig. 11A). Haptomère formant une saillie sclérifiée redressée. Aire pédiale glabre, traversée par un sillon sinueux. Chaetoparia dissymétriques à rangs concentriques de soies convergeant vers l'aire pédiale, plus épaisses en avant et à l'intérieur, plus fines à la périphérie. Acanthoparia avec 12 à 15 courtes soies lamelliformes incurvées vers l'avant. Corypha composé de soies épaisses.

Mandibules (Fig. 11E-H). Aire scissoriale de la mandibule gauche avec une forte dent apicale pointue, les deuxième et troisième dents petites et saillantes (Fig. 11E, F). Aire scissoriale de la mandibule droite avec une forte dent apicale pointue et une seconde dent arrondie saillante (Fig. 11G, H). 3 à 4 longues soies +1 ou 2 courtes soies latéroexternes. Sur la face ventrale des 2 mandibules, présence d'une aire stridulatoire composée de 19 à 20 bourrelets parallèles.
Maxilles (Fig. 16M). Galea et lacinia soudées. Lacinia à uncus avec 3 dents de longueurs très inégales (2 grandes et 1 très petite), sclérifiées, émoussées et soudées à la base. Galea avec uncus sclérifié. Sur la face dorsale, aire stridulatoire à $7+1$ dents à sommet émoussé.

Hypopharynx (Fig. 10G). Une touffe de longues soies de chaque côté, à la base des palpes; soies plus courtes et plus fortes au milieu et sur la partie basale. Oncyli transverses, avec une dent sclérifiée postérieure subtriangulaire émoussée et une très forte dent antérieure, sclérifiée et dressée.

\section{Thorax}

Pattes (Fig. 19C). Longues, de longueur croissante de $\mathrm{P} 1$ à $\mathrm{P} 3$, toutes de structure analogue: coxa, trochanter, fémur, tibiotarse, griffe forte, longue, aiguë, incurvée et sclérifiée à l'apex.

\section{Abdomen}

Raster (Fig. 3H). Fente anale transverse, légèrement incurvée, éloignée de l'apex abdominal. Du côté dorsal de la fente anale, soies très courtes et légèrement incurvées, sans orientation préférentielle, peu nombreuses et dispersées. Le long du bord ventral de la fente anale, une rangée de longues et fortes soies dressées. Teges constitués d'épines plus grosses et plus longues que du côté dorsal, incurvées, orientées vers l'arrière. Pas de bande glabre le long de la fente anale. Barbula avec de longues soies dispersées.

Hexodon unicolor (Olivier, 1789)

(Figs $2 \mathrm{H} ; 4 \mathrm{~B} ; 6 \mathrm{~B} ; 7 \mathrm{G} ; 10 \mathrm{H} ; 12 \mathrm{H}$;

$14 \mathrm{~T}-\mathrm{W} ; 16 \mathrm{~N} ; 19 \mathrm{D} ; 21 \mathrm{R}-\mathrm{T} ; 24 \mathrm{~N}, \mathrm{O})$

\section{MATÉRIEL EXAMINÉ}

Larves provenant d'Andranomanelatra, prélevées dans une parcelle menée en système de culture à base de Riz pluvial en haut de pente. La larve est indifférente à la culture et améliore la structure du sol (Ratnadass et al. 2006). Type de sol: ferrallitique. Spécimens conservés dans les collections du Fofifa (6 larves), et du Cirad-CBGP (1 larve).

\section{DESCRIPTION}

Couleur blanc ivoire (Fig. 2H). 
Tête (Fig. 6B)

Couleur brunâtre. Épicrâne avec 2 soies dorsoépicraniales et 2 soies épicraniales latéro-externes disposées symétriquement. Une soie sur chaque côté de l'angle frontal. Clypéus transverse avec une soie clypéale externe sur chaque côté.

Antennes (Fig. 12H). Article II approximativement deux fois plus long que les autres (les longueurs respectives des articles sont: $\mathrm{I}=1,14$; II $=2,14$; $\mathrm{III}=1,28 ; \mathrm{IV}=1$ ). Une aire sensorielle apico-latérale sur l'avant-dernier article. Dernier article antennaire avec 5 plages sensorielles: 1 petite plage apicale ronde, 2 ventrales écartées et 2 dorsales adjacentes.

Labre. Transverse, asymétrique. Zone médiane du bord antérieur un peu saillante, formant presque un lobe médian, légèrement différencié du côté droit, avec 4 soies antérieures externes et 2 soies antérieures internes écartées. De part et d'autre, 1 soie latéro-antérieure externe, 2 soies latéro-antérieures internes, puis 1 longue soie latérale. 2 longues soies labro-internes. 4 soies postérieures (en 2 groupes transverses de 2 soies).

Épipharynx (Fig. 7G). Haptomère formant une saillie redressée bifide, sclérifiée et émoussée. Acroparia avec de fortes soies. Aire pédiale glabre, avec un sillon anguleux, en arc de cercle vers la base. Chaetoparia dissymétriques à rangs concentriques de soies convergeant vers l'aire pédiale, plus fortes près de l'haptomère et sur la périphérie de l'aire pédiale, plus fines vers l'extérieur. Acanthoparia avec 6 à 9 courtes soies lamelliformes incurvées vers l'avant.

Mandibules (Fig. 14T-W). Aire scissoriale de la mandibule gauche avec une forte dent apicale, suivie d'une dent arrondie et d'une petite dent pointue. Aire scissoriale de la mandibule droite avec une forte dent apicale, suivie d'une forte dent arrondie. Mandibules gauche et droite avec chacune 6 à 8 soies baso-latérales et 2 soies dorso-externes. Sur la face ventrale des 2 mandibules, présence d'une aire stridulatoire formée de 35 bourrelets parallèles transverses.

Maxilles (Fig. 16N). Galea et lacinia soudées. Lacinia à uncus avec 2 dents. Galea à uncus sclérifié. Sur la face dorsale, aire stridulatoire composée de 9 à 10 dents irrégulières aux bords arrondis émoussés.

Hypopharynx (Fig. 10H). Glosses avec une vingtaine à une trentaine de courtes et fortes soies au milieu et à la base, ainsi qu'avec des touffes de longues soies vers l'apex et sur les côtés. Oncyli transverses, avec une dent sclérifiée postérieure subtriangulaire et une forte dent antérieure, sclérifiée et dressée.

\section{Thorax}

Pattes (Fig. 19D). Longues, de longueur croissante de P1 à P3, toutes de structure analogue: coxa, trochanter, fémur, tibiotarse, griffe forte, longue, aiguë, anguleuse à la base, incurvée et sclérifiée à l'apex.

\section{Abdomen}

Raster (Fig. 4B). Du côté dorsal de la fente anale transverse, nombreuses soies courtes et épaisses, orientées vers l'avant, mêlées de quelques longues soies plus fines. Une étroite bande glabre le long de la fente anale du côté dorsal. Teges avec de nombreuses et très épaisses soies aplaties et recourbées à l'extrémité du côté ventral de la fente anale. Barbula avec de rares et longues soies.

Heteroconus paradoxus (Endrödi, 1968)

(Figs 2I ; 4D; 8A; 10I ; 12I ; 14X-Z $16 \mathrm{O} ; 19 \mathrm{E} ; 21 \mathrm{U}-\mathrm{W} ; 25 \mathrm{~N}-\mathrm{P})$

\section{MATÉRIEL EXAMINÉ}

Larves provenant d'Ankevobe (13 km d'Antsirabe) et d'Ampandrotrarana (20 km d'Antsirabe) dans des cultures de Riz pluvial (Oryza sativa) et d'Arachide (Arachis hypogea L.) en haut de pente. Type de sol: ferrallitique. Spécimens conservés dans les collections du Fofifa (20 larves), du Cirad-CBGP (3 larves) et du MNHN (2 larves).

\section{DESCRIPTION}

Couleur blanc ivoire (Fig. 2I).

\section{Tête}

Couleur brun jaunâtre. Épicrâne avec 6 soies dorsoépicraniales et 6 soies épicraniales latéro-externes 
disposées symétriquement. 5 soies de chaque côté de l'angle frontal. 2 soies frontales antérieures disposées symétriquement. Clypéus transverse avec 5 soies clypéales externes et 1 soie interne de chaque côté.

Antennes (Fig. 12I). Les longueurs respectives des articles sont: $\mathrm{I}=1,75 ; \mathrm{II}=1,91 ; \mathrm{III}=1,58 ; \mathrm{IV}=1$. Une aire sensorielle apico-latérale sur l'avant-dernier article. Dernier article antennaire avec 4 plages sensorielles: 1 petite plage apicale ronde, 2 ventrales et 1 dorsale. 7 longues soies sur l'article I et 3 longues soies sur l'article II.

Labre. Transverse, asymétrique, avec de longues soies. Zone médiane du bord antérieur saillante, formant presque un lobe médian, légèrement différencié du côté droit, avec 4 soies antérieures externes et 2 soies antérieures internes rapprochées. De part et d'autre, 3 à 4 soies latéro-antérieures externes, 1 à 2 soies latéro-antérieures internes, puis 1 soie latérale. 2 longues soies labro-internes en avant du milieu. Une rangée irrégulière de 15 soies postérieures en 2 groupes de 8 et 7 , la zone centrale étant glabre.

Épipharynx (Fig. 8A). Haptomère formant une saillie redressée sclérifiée arrondie. Acroparia avec de fortes soies très denses. Aire pédiale glabre, avec un sillon anguleux, en arc de cercle vers la base. Chaetoparia dissymétriques, à longues et fortes soies convergeant vers l'aire pédiale, les plus fortes soies étant à proximité de l'haptomère. Acanthoparia avec 21 à 22 soies lamelliformes, de taille croissante, incurvées vers l'avant.

Mandibules (Fig. 14X-Z $Z_{2}$ ). Aire scissoriale de la mandibule gauche avec 3 dents développées, la dent apicale très forte et tronquée, la seconde dent arrondie et la troisième aiguë. Aire scissoriale de la mandibule droite avec une forte dent apicale émoussée, la seconde dent arrondie. 19 à 20 soies soies baso-latérales et 3 soies dorso-externes. Face ventrale des deux mandibules avec une aire stridulatoire proche du lobe molaire et d'une touffe de courtes soies, très réduite, difficile à observer, avec seulement 4 à 5 bourrelets transverses, subconvergents, espacés.
Maxilles (Fig. 16O). Galea et lacinia soudées. Lacinia à uncus avec deux dents sclérifiées soudées à la base. Galea avec uncus sclérifié. Sur la face dorsale, aire stridulatoire composée de 12 à 15 dents à sommet aplati.

Hypopharynx (Fig. 10I). Glosses recouvertes au milieu et à la base par des soies courtes, fortes et pointues disposées en triangle et par de longues soies sur le reste de la surface.

\section{Thorax}

Pattes (Fig. 19E). Longues, de longueur croissante de P1 à P3, toutes de structure analogue: coxa, trochanter, fémur, tibiotarse, griffe longue, aiguë, incurvée et sclérifiée à l'apex. Coxa 3 très longue.

\section{Abdomen}

Raster (Fig. 4D). Fente anale transverse. Une étroite bande glabre le long du côté dorsal de la fente anale. Du côté dorsal de la fente anale, nombreuses soies courtes et épaisses avec quelques très longues soies fines. Six longues soies du côté ventral de la fente anale. Teges avec plus d'une trentaine de très fortes soies recourbées et aplaties.

Paranodon coquerelii (Fairmaire, 1871)

(Figs 4E; 8B ; 10J ; 12J ; 15A-D; 17A; 19F; 22A-C; 24P, Q)

\section{MATÉRIEL EXAMINÉ}

Larves provenant d'Ampandrotrarana $(20 \mathrm{~km}$ d'Antsirabe) dans une culture de Riz pluvial en haut de pente. Type de sol: ferrallitique. Spécimens conservés dans les collections du Fofifa (1 larve), du Cirad-CBGP (1 larve) et du MNHN (1 larve).

\section{DESCRIPTION}

Couleur blanche.

\section{Tête}

Couleur jaune. Épicrâne avec 3 soies dorso-épicraniales et 4 soies latéro-externes disposées symétriquement. Front avec 2 soies dans l'angle frontal, 1 soie frontale postérieure et 2 soies antérieures disposées symétriquement. Clypéus transverse avec 
2 soies clypéales postérieures et 2 soies clypéales externes de chaque côté.

Antennes (Fig. 12J). Les longueurs respectives des articles sont: $\mathrm{I}=1,36 ; \mathrm{II}=1,54 ; \mathrm{III}=1,27 ; \mathrm{IV}=1$. Une aire sensorielle apico-latérale sur l'avant-dernier article. Dernier article antennaire avec 5 plages sensorielles : 1 petite plage apicale ronde, 2 ventrales écartées et 2 dorsales adjacentes.

Labre. Transverse, asymétrique, avec de longues soies. Zone médiane du bord antérieur non-saillante, différenciée du côté droit, avec 4 soies antérieures externes et 2 soies antérieures internes rapprochées. De part et d'autre, 5 soies latéro-antérieures externes et 2 soies latéro-antérieures internes, puis 1 soie latérale. 2 longues soies labro-internes en avant du milieu. Une rangée transverse de 7 soies postérieures en 2 groupes de 4 et 3 soies, la zone centrale étant glabre.

Épipharynx (Fig. 8B). Haptomère à bord relevé, sclérifié et émoussé. Clithrum sclérifié. Aire pédiale très petite, glabre. Chaetoparia dissymétriques, à fortes soies épaisses convergeant vers l'aire pédiale. Acanthoparia avec 9 courtes soies incurvées lamelliformes, de taille croissante vers l'avant.

Mandibules (Fig. 15A-D). Aire scissoriale de la mandibule gauche avec 4 dents, l'apicale forte, la deuxième arrondie et très émoussée, la troisième et la quatrième pointues et plus écartées que les autres. 5 soies latéro-externes et 2 soies dorso-externes. Aire scissoriale de la mandibule droite à 3 dents, l'apicale pointue, la deuxième arrondie et la troisième émoussée en angle obtus. 5 soies latéro-externes et 2 soies dorso-externes. Sur la face ventrale des 2 mandibules, présence d'une aire stridulatoire composée de 23 à 26 bourrelets parallèles.

Maxilles (Fig. 17A). Galea et lacinia soudées. Lacinia à uncus avec 4 dents (3 grandes et 1 petite). Galea avec uncus sclérifié. Sur la face dorsale, aire stridulatoire composée de 9 à 10 dents crénelées.

Hypopharynx (Fig. 10J). Surface entre la base des palpes et les oncyli couverte de longues et fortes soies. Oncyli transverses, avec une dent sclérifiée postérieure très émoussée et une forte dent antérieure, sclérifiée et dressée.

\section{Thorax}

Pattes (Fig. 19F). Longues, de longueur croissante de P1 à P3, toutes de structure analogue: coxa, trochanter, fémur, tibiotarse, griffe longue, aiguë, incurvée, sclérifiée.

\section{Abdomen}

Raster (Fig. 4E). Fente anale transverse. Une bande glabre du côté dorsal de la fente anale, barbula composée de nombreuses longues soies fines. Une rangée de longues soies du côté ventral de la fente anale. Teges avec une trentaine de soies épaisses, aplaties et incurvées, avec de chaque côté une très forte et épaisse épine dissymétrique (trifide ou bifide du côté gauche et bifide ou simple du côté droit), séparées par une zone glabre.

Famille HOPLIIDAE Latreille, 1829

Paramorphochelus cornutus (Nonfried, 1892) (Figs 2J; 4F; 6D; 8C; 10K; 13C;

15E-H; 17B; 18E; 22D, E; 24K-M)

\section{MATÉRIEL EXAMINÉ}

Larves provenant d'Andranomanelatra et d'Ivory dans une parcelle de Riz pluvial. Type de sol: ferrallitique. Spécimens conservés dans les collections du Fofifa (7 larves), du Cirad-CBGP (8 larves) et du MNHN (4 larves).

\section{DESCRIPTION}

Couleur blanche (Fig. 2J).

\section{Tête (Fig. 6D)}

Couleur jaune. 1 soie dorso-épicraniale et 1 soie latéro-externe disposées symétriquement. 1 soie frontale postérieure et 2 soies frontales antérieures disposées symétriquement. Clypéus avec 1 soie latérale externe et 1 soie antérieure à disposition symétrique.

Antennes. Les longueurs respectives des articles sont: $\mathrm{I}=1 ; \mathrm{II}=2 ; \mathrm{III}=1,36 ; \mathrm{IV}=1,27$. Dernier article 
antennaire avec 4 plages sensorielles : 2 ventrales, 1 minuscule plage apicale et 1 dorsale. 1 longue soie sur le second article. Avant-dernier article avec 1 saillie apico-latérale.

Labre (Fig. 13C). Transverse, symétrique, faiblement trilobé. Lobe médian avec 6 soies insérées chacune sur une protubérance pigmentée, les deux protubérances médianes accolées, presque fusionnées. Lobe latéral avec 2 soies externes et 2 soies internes, insérées chacune sur une protubérance pigmentée. 1 soie latéro-antérieure. 1 soie latérale. 2 longues soies labro-internes en avant du milieu. Une rangée transverse irrégulière de 4 à 5 soies postérieures.

Épipharynx (Fig. 8C). Haptomère formé par 9 à 11 fortes épines disposées en fer à cheval. Aire pédiale large et glabre. Chaetoparia dissymétriques, les soies convergeant vers l'aire pédiale. Gymnoparia très larges. Acanthoparia avec 6 à 9 courtes soies lamelliformes incurvées vers l'avant.

Mandibules (Fig. 15E-H). Aire scissoriale de la mandibule gauche allongée, se terminant par 3 dents, l'apicale et la troisième dents aiguës, la deuxième dent émoussée. Aire scissoriale de la mandibule droite allongée, avec 2 dents émoussées. 7 soies latéro-externes et 1 soie dorso-externe. Il n'existe pas d'aire stridulatoire sous la forme d'une aire striée en vis-à-vis des dents stridulatoires maxillaires.

Maxilles (Fig. 17B). Galea et lacinia soudées. Lacinia à uncus avec trois dents inégales et une très forte épine, galea à uncus pointu sclérifié et 6 fortes épines. Sur la face dorsale, 10 à 11 dents stridulatoires, séparées, pointues et inclinées vers l'apex.

Hypopharynx (Fig. 10K). Glosses avec de très longues soies à l'apex et de plus courtes et plus fortes soies à la base. Oncyli transverses, avec une dent sclérifiée postérieure triangulaire et une forte dent antérieure, sclérifiée et dressée.

\section{Thorax}

Pattes (Fig. 18E). Longues, de taille croissante de P1 à P3. Coxa très allongée à apex arrondi, trochanter inséré latéralement sur la coxa, fémur, tibiotarse avec de fortes soies mêlées à de longues soies à l'apex. Tibiotarse 3 plus court, plus large et plus arrondi que les deux précédents, sans griffe. Apex du tibiotase 1 avec une griffe sclérifiée large à la base, terminée par une longue pointe aiguë. Apex du tibiotase 2 avec une griffe sclérifiée plus courte que la précédente, large et anguleuse à la base, terminée en pointe.

\section{Abdomen}

Raster (Fig. 4F). Fente anale en "Y». Présence tout le long du côté dorsal comme du côté ventral de la fente anale de courtes soies mêlées à d'autres plus longues. Teges avec une quarantaine de fortes soies aplaties incurvées à l'apex. Barbula de longues soies très denses.

\section{Famille Melolonthidae MacLeay, 1819}

Encya sikorai (Brenske, 1891)

(Figs 2K; 4H; 8E; 10L; 11C, D; 12K-M;

15I-L; 17C; 20A; 22F-K; 23J)

\section{MATÉRIEl eXAminÉ}

Larves provenant d'Andranomanelatra, de Bevalala, d'Ivato et de Manjakandriana dans une jachère de Cynodon dactylon et de Graminées diverses, dans une culture de Pennisetum purpureum. Elles sont fréquemment trouvées en milieu humide en bordure de rizière et près des marais. Types de sol: alluvionnaire, ferrallitique, argileux, de couleur noire, riche en matière organique. Spécimens conservés dans les collections du Fofifa (5 larves), du Cirad-CBGP (1 larve) et du MNHN (2 larves).

Dans une parcelle de Riz pluvial, les larves de cette espèce avaient coupé les racines des plants et endommagé plus de $80 \%$ des plants. Les attaques ont eu lieu à partir de la fin du tallage de la culture.

\section{DESCRIPTION}

Couleur blanc laiteux. Pattes longues (Fig. 2K).

Tête

Tête brun jaunâtre. 3 courtes soies dorso-épicraniales disposées symétriquement de chaque côté de la capsule céphalique. 
Antennes (Fig. 12K-M). Les longueurs respectives des articles sont: $\mathrm{I}=\mathrm{II}=\mathrm{III}=1,36$; IV $=1,84$. Dernier article antennaire fusiforme avec 5 plages sensorielles: 3 ventrales, 1 petite apicale et 1 dorsale (Fig. 12L, M). Avant-dernier article avec une saillie apico-latérale.

Labre. Un peu plus large que long, symétrique. Zone médiane du bord antérieur nettement saillante, avec 2 soies externes rapprochées et 4 soies internes. 2 à 3 soies latéro-antérieures et 1 soie latérale. Soies de l'acanthoparia bien visibles latéralement, de l'aire latéro-antérieure à l'aire latéro-postérieure. 2 soies labro-internes.

Épipharynx (Fig. 8E). Haptomère constitué d'un groupe de fortes épines très sclérifiées, subégales. Aire pédiale glabre. Proplegmatum très développé, rétréci vers l'avant, formé de 12 à 15 bourrelets parallèles. Plegmatum présent. Acanthoparia formés de 13 à 16 courtes soies lamelliformes de taille croissante vers l'avant. Dexiophobe constitué d'une rangée de courtes soies parallèles, laeophobe constitué d'une rangée de soies parallèles un peu plus longues. Chaetoparia assymétriques, à rangs concentriques d'épaisses et fortes soies à l'avant, plus fines et courtes à l'arrière, plus fortes du côté du dexiophobe, plus fines et allongées du côté du laeophobe, convergeant vers l'aire pédiale.

Mandibules (Fig. 15I-L). Pas d'aire stridulatoire. Aire scissoriale de la mandibule gauche allongée, avec une très forte dent apicale et une seconde dent triangulaire; aire molaire gauche avec une très forte dent. Aire scissoriale de la mandibule droite allongée, avec une très forte dent apicale et une seconde dent triangulaire obtuse. 7 soies latéro-externes, 1 soie dorso-externe et une touffe de 4 à 7 courtes soies dorso-molaires.

Maxilles (Fig. 17C). Galea et lacinia fusionnées à la base et séparées à l'apex. Lacinia à uncus tridenté sclérifié, armée de 4 fortes épines sclérifiées. Galea à uncus sclérifié, armée de 5 ou $6+2$ ou 3 fortes épines sclérifiées. Sur la face dorsale, aire stridulatoire composée de 17 à 21 dents sclérifiées saillantes formant des créneaux émoussés.
Hypopharynx (Fig. 10L). Glosses recouvertes vers la base des palpes de très longues soies mêlées à des soies très courtes, orientées vers l'apex. En avant des oncyli, une aire subtriangulaire médiane constituée de soies plus fortes orientées vers la base. Oncyli transverses, sclérifiés dans la partie centrale.

\section{Thorax}

Pattes (Fig. 20A). Longues, de longueur croissante de P1 à P3, toutes de structure analogue: coxa, trochanter inséré latéralement sur la coxa, fémur, tibiotarse. $\mathrm{P} 1$ et $\mathrm{P} 2$ à griffe très longue, très forte à la base, incurvée, sclérifiée à l'apex. $\mathrm{P} 3$ à griffe très courte, plus large que longue, sclérifiée sur une étroite bande apicale, portant 2 longues soies.

\section{Abdomen}

Raster (Fig. 4H). Fente anale en "Y», bordée de soies des deux côtés. Palidia formés de 2 groupes de fortes soies, enchevêtrées et orientées vers la septula, qui est glabre. Teges périphériques étroits, constitués de courtes soies recourbées. Barbula constituée de longues soies.

Enaria melanictera (Klug, 1833)

(Figs 5A; 8F ; 12A; 13D; 15M-P;

$17 \mathrm{D} ; 20 \mathrm{~B} ; 22 \mathrm{~L}-\mathrm{N} ; 23 \mathrm{~K}-\mathrm{M})$

\section{Matériel EXAMINÉ}

Larves provenant d'Antanikatsaka (5 km d'Antsirabe), de Sakay dans des cultures de Maïs (Zea mays) et de Riz pluvial (Oryza sativa). Type de sol: ferrallitique. Spécimens conservés dans les collections du Fofifa (6 larves), du Cirad-CBGP (11 larves) et du MNHN (10 larves).

\section{DESCRIPTION \\ Couleur blanche.}

\section{Tête}

De couleur jaunâtre. Front avec 7 soies frontales antérieures, 1 soie frontale externe et 3 soies frontales postérieures disposées symétriquement. 1 soie de chaque côté de l'angle frontal. Épicrâne avec 6 soies dorso-épicraniales et 4 soies latéro-externes. 
Antennes. Les longueurs respectives des articles sont: $\mathrm{I}=2,09 ; \mathrm{II}=1,63$; III $=1,54 ; \mathrm{IV}=1$. Deuxième article avec 2 longues soies, le troisième article avec 1 soie. Dernier article antennaire avec 4 plages sensorielles: 2 ventrales, 1 petite apicale et 1 dorsale. Avant-dernier article avec une saillie ventrale apico-latérale.

Labre (Fig. 13D). Un peu plus large que long, symétrique. Zone médiane du bord antérieur en pointe arrondie, avec $2+2$ soies externes et 4 soies internes rapprochées. 2 soies latéro-antérieures externes et 2 + 1 soies latéro-antérieures internes, 1 soie latérale et une à 4 courtes soies latéro-postérieures. Soies de l'acanthoparia bien visibles latéralement, de l'aire latéro-antérieure à l'aire latéro-postérieure. 2 soies labro-internes.

Épipharynx (Fig. 8F). Haptomère constitué d'une rangée transversale de 5 très fortes épines sclérifiées subégales. Proplegmatum peu développé. Plegmatum bien distinct, s'élargissant vers l'avant. Acanthoparia formé de 13 à 15 soies lamelliformes incurvées vers l'avant. Chaetoparia dissymétriques, les soies convergeant vers l'aire pédiale, les plus petites à la périphérie. Aire pédiale glabre.

Mandibules (Fig. 15M-P). Pas d'aire stridulatoire. Aire scissoriale de la mandibule gauche allongée, la dent apicale longue et émoussée, la seconde très effacée, la troisième émoussée. Aire scissoriale de la mandibule droite allongée, la dent apicale émoussée, suivie par une dent arrondie très émoussée. Aire molaire de la mandibule gauche avec une forte dent subcarrée. 6 longues soies latéro-externes et 1 soie dorso-externe.

Maxilles (Fig. 17D). Galea et lacinia soudées à la base et séparées à l'apex. Lacinia à uncus avec 3 dents développées à apex tronqué, avec une rangée de 5 à 6 épines. Galea à uncus sclérifié, armée de 8 fortes épines sclérifiées. Sur la face dorsale, aire stridulatoire composée de 11 à 13 dents sclérifiées, rapprochées, coniques, inclinées vers l'apex.

Hypopharynx (Fig. 12A). Glosses couvertes sur les côtés et vers la base des palpes de longues soies, mêlées à des soies très courtes, orientées vers l'apex. Soies très fortes et denses en avant des oncyli, lesquels sont transverses, sclérifiés dans la partie centrale.

\section{Thorax}

Pattes (Fig. 20B). Longues, de longueur croissante de P1 à P3, toutes de structure analogue: coxa, trochanter inséré latéralement sur la coxa, fémur, tibiotarse. P1 et P2 à griffe longue, forte à la base, incurvée, sclérifiée à l'apex. P3 à griffe très courte, plus large que longue, portant deux longues soies.

\section{Abdomen}

Raster (Fig. 5A). Fente anale en "Y" bordée de courtes soies. Palidia en " $V$ » inversé, formés de 10 à 12 soies épineuses, plus longues vers l'apex. Teges formés de 4 rangées de courtes et fortes soies sclérifiées, aplaties et incurvées. Barbula avec de nombreuses et denses longues soies.

$$
\begin{aligned}
& \text { Apicencya waterloti (Dewailly, 1950) } \\
& \text { (Figs 2L; 5C; 9A; 12B, N; 15Q-T; } \\
& \text { 17E; 20C; 22O-Q; 24A-I) }
\end{aligned}
$$

\section{MATÉRIEL EXAMINÉ}

Larves provenant d'Andranomanelatra, dans une culture de Riz pluvial située en haut de pente. Elles coupaient les racines des plants, avec une destruction à plus de $90 \%$. Type de sol: ferrallitique. Spécimens conservés dans les collections du Fofifa (14 larves), du Cirad-CBGP (1 larve) et du MNHN (1 larve).

\section{DESCRIPTION}

Couleur blanche (Fig. 2L).

\section{Tête}

Couleur jaunâtre. Épicrâne avec symétriquement 7 à 9 soies latéro-externes; 2 soies dorso-épicraniales. Front avec 9 soies frontales antérieures, 2 soies frontales externes et 4 soies frontales postérieures, disposées symétriquement. Clypéus avec 1 soie clypéale externe et 2 soies clypéales antérieures symétriques.

Antennes (Fig. 12N). Les trois premiers articles subégaux, le quatrième plus court (longueurs res- 
pectives des articles: $\mathrm{I}=\mathrm{III}=1,93 ; \mathrm{II}=2 ; \mathrm{IV}=1)$. Dernier article antennaire avec 4 plages sensorielles: 2 ventrales, 1 petite aire apicale ronde et 1 dorsale, l'article I portant 4 soies et l'article II portant 2 soies. Avant-dernier article avec une saillie ventrale apico-latérale.

Labre. Un peu plus large que long, symétrique. Zone médiane du bord antérieur en pointe arrondie, avec $2+2$ soies externes et quatre soies internes rapprochées à l'intérieur d'une zone pigmentée subcirculaire. 2 soies latéro-antérieures externes et $2+1$ soies latéro-antérieures internes, 1 soie latérale et 5 à 7 courtes soies latéro-postérieures. Soies de l'acanthoparia bien visibles latéralement, de l'aire latéro-antérieure à l'aire latéro-postérieure. 2 soies labro-internes.

Épypharynx (Fig. 9A). Haptomère constitué d'un groupe de très fortes épines. Proplegmatum réduit, formé de 12 à 15 bourrelets parallèles. Plegmatum développé. Acanthoparia avec 13 à 16 courtes soies lamelliformes incurvées vers l'avant, de taille croissant d'arrière en avant. Chaetoparia dissymétriques, à fortes soies convergeant vers l'aire pédiale, qui est glabre.

Mandibules (Fig. 15Q-T). Pas d'aire stridulatoire. Aire scissoriale de la mandibule gauche allongée, avec une forte dent apicale émoussée, la deuxième dent arrondie et la troisième dent fine peu saillante. Aire scissoriale de la mandibule droite allongée, avec une forte dent apicale émoussée, une seconde dent effacée et une troisième dent arrondie. 8 soies latéro-externes et 1 soie dorso-externe.

Maxilles (Fig. 17E). Galea et lacinia soudées à la base, séparées à l'apex. Lacinia à uncus avec 3 dents sclérifiées développées, entourées par une rangée de $4+2$ épines de chaque côté. Galea avec uncus sclérifié, armée de $6+2$ fortes épines sclérifiées. Sur la face dorsale, aire stridulatoire composée de 16 à 17 dents séparées en forme de cônes sclérifiés.

Hypopharynx (Fig. 12B). Glosses densément recouvertes de longues soies à l'apex et de très fortes soies moins denses orientées vers l'arrière et formant une zone subtriangulaire à la base.

\section{Thorax}

Pattes (Fig. 20C). Longues, de longueur croissante de P1 à P3, toutes de structure analogue: coxa, trochanter inséré latéralement sur la coxa, fémur, tibiotarse. P1 et $\mathrm{P} 2$ à griffe très longue, très forte à la base, incurvée, sclérifiée à l'apex. P3 à griffe courte, à peine plus longue que large, à apex pointu sclérifié, portant deux longues soies.

\section{Abdomen}

Raster (Fig. 5C). Fente anale en «Y». Palidia en forme de fuseau, formés de deux rangées symétriques de 13-15 fortes soies orientées vers l'intérieur. Septula glabre. Teges formés de soies concentriques, denses, épaisses et longues à l'intérieur, plus courtes et éparses à l'extérieur. Barbula avec de nombreuses soies allongées.

\section{Empecta scutata (Fairmaire, 1901)}

(Figs 2M; 5D; 6E; 9B; 12C, O;

15U-X; 17F; 20D; 22R-T; 24J)

\section{MATÉRIEL EXAMINÉ}

Larves provenant d'Ivato, dans une parcelle avec Cynodon dactylon, Pennisetum purpureum. L'espèce a été collectée dans des parcelles en bas de pente situées près des rizières et en haut de pente sur milieu sec. Types de sol: alluvionnaire, de couleur noire, riche en matière organique. Spécimens conservés dans les collections du Fofifa (4 larves) et du Cirad-CBGP (1 larve).

\section{DESCRIPTION}

De couleur blanc laiteux (Fig. 2M).

Tête (Fig. 6E)

Couleur jaunâtre. Épicrâne avec 5 soies latéroexternes alignées, disposées symétriquement, 2 soies dorso-épicraniales. Front avec 6 soies frontales antérieures, 1 soie frontale externe et 2 soies frontales postérieures disposées symétriquement. Présence d'une soie de chaque côté de l'angle frontal. Clypéus avec 1 soie clypéale externe et 2 soies clypéales antérieures symétriques.

Antennes (Fig. 12O). Les trois premiers articles subégaux, le quatrième plus court (longueurs 
respectives des articles: $\mathrm{I}=1,46 ; \mathrm{II}=1,61$; III $=$ 1,$69 ; \mathrm{IV}=1$ ). Dernier article antennaire avec 4 plages sensorielles: 2 ventrales, 1 petite aire apicale ronde et 1 dorsale, l'article I portant 2 soies et l'article II portant 1 soie. Avant-dernier article avec une saillie ventrale apico-latérale.

Labre. Un peu plus large que long, symétrique, avec des soies de section non circulaire, aplaties. Zone médiane du bord antérieur en pointe, avec $2+2$ soies externes et 4 soies internes rapprochées (celles en position centrale plus fortes que celles en position périphérique). 2 soies latéro-antérieures externes et $2+1$ soies latéro-antérieures internes. 1 soie latérale. Soies de l'acanthoparia bien visibles latéralement, de l'aire latéro-antérieure à l'aire latéro-postérieure. 2 soies labro-internes. Une rangée transversale irrégulière d'une quinzaine de soies postérieures.

Épipharynx (Fig. 9B). Haptomère avec une rangée transverse de 7 très fortes épines. Acanthoparia avec 8 à 11 soies lamelliformes incurvées vers l'avant. Proplegmatum absent. Plegmatum bien développé, sa plus grande largeur au milieu. Chaetoparia plus ou moins symétriques, à rangs concentriques de soies de densité moyenne convergeant vers l'aire pédiale, qui est glabre.

Mandibules (Fig. 15U-X). Pas d'aire stridulatoire. Aire scissoriale de la mandibule gauche allongée, avec une forte dent apicale très émoussée, une seconde dent obtuse et une troisième dent pointue mais émoussée. Aire scissoriale de la mandibule droite allongée, avec une forte dent apicale, une deuxième dent effacée et la troisième pointue mais émoussée. 13 à 14 longues soies latéro-externes.

Maxilles (Fig. 17F). Galea et lacinia soudées à la base et séparées à l'apex. Lacinia à uncus avec 3 fortes dents sclérifiées, alignées et émoussées, avec 4 soies épineuses sclérifiées de taille décroissante de l'apex vers la base. Galea à uncus sclérifié, émoussé, avec 6 très fortes soies épineuses sclérifiées du côté inférieur et 4 fortes soies sclérifiées de taille décroissante de l'apex vers la base du côté supérieur. Sur la face dorsale, aire stridulatoire composée de 13 à 14 dents séparées inclinées vers l'apex.
Hypopharynx (Fig. 12C). Glosses couvertes de longues soies (parmi lesquelles 4 sont très fortes) vers l'apex et de plus d'une vingtaine de fortes soies épineuses éparses à la base. Oncyli transverses, avec une forte dent sclérifiée tranchante.

\section{Thorax}

Pattes (Fig. 20D). Longues, de longueur croissante de P1 à P3, de structure analogue: coxa, trochanter inséré latéralement sur la coxa, fémur, tibiotarse. P1 et P2 à griffe longue, sublinéaire, large à la base, pointue, sclérifiée à l'apex, la griffe 1 plus longue que la 2. Tibiotarse 3 à apex arrondi, sans griffe.

\section{Abdomen}

Raster (Fig. 5D). Fente anale en «Y». Palidia formés vers l'avant de 2 rangées symétriques de très courtes épines, et vers l'arrière de 2 groupes symétriques de fortes et longues épines aplaties, l'ensemble dessinant un «T» inversé, toutes les soies inclinées vers le septula, qui est glabre. Teges formés de courtes soies peu nombreuses et dispersées, disposées symétriquement de chaque côté des palidia. Barbula avec de longues soies peu nombreuses. Du côté dorsal de la fente anale, courtes et longues soies mêlées.

\author{
Hoplochelus betanimena (Künckel, 1887) \\ (Figs 2N; 5E; 9C; 12D; 15Y-Z ; \\ $17 \mathrm{G} ; 20 \mathrm{E} ; 22 \mathrm{U}-\mathrm{W} ; 23 \mathrm{I})$
}

\section{MATÉRIEL EXAMINÉ}

Larves provenant de Bevalala et d'Ivato (dans des parcelles de Pennisetumpurpureum) et de Manjakandriana (dans une jachère envahie par diverses Graminées, dans une zone de riziculture). Fréquemment trouvée en milieu humide en bas de pente (près de marais, en bordure de rivière). Types de sol: alluvionnaire, argileux, de couleur noire, riche en matière organique. Spécimens conservés dans les collections du Fofifa (1 larve) et du Cirad-CBGP (1 larve).

\section{DESCRIPTION}

De couleur blanche (Fig. 2N). 
Tête

Couleur jaunâtre. Épicrâne avec, symétriquement, 1 courte soie dorso-épicraniale et 3 soies latéroexternes. Clypéus avec 1 soie clypéale externe et 2 soies clypéales antérieures symétriques.

Antennes. Longueurs respectives des articles: $\mathrm{I}=1,66$; II $=$ III $=2,06 ; \mathrm{IV}=1$. Dernier article antennaire avec 4 plages sensorielles: 2 ventrales, 1 petite aire apicale ronde et 1 dorsale, l'article I portant 3 soies et l'article II portant 1 soie. Avant-dernier article avec une saillie ventrale apico-latérale.

Labre. Transverse, subsymétrique. Zone médiane du bord antérieur en pointe émoussée, avec 2 + 2 soies externes et 4 soies internes rapprochées. 3 soies latéro-antérieures externes et $2+1$ soies latéro-antérieures internes. 1 soie latérale. Soies de l'acanthoparia bien visibles latéralement, de l'aire latéro-antérieure à l'aire latéro-postérieure. 2 soies labro-internes.

Épipharynx (Fig. 9C). Haptomère constitué de 2 rangées de 7 et 6 fortes épines coniques sclérifiées implantées sur un relief saillant. Acanthoparia avec 13 à 15 soies lamelliformes incurvées vers l'avant. Proplegmatum absent. Plegmatum développé, sa plus grande largeur au milieu. Chaetoparia presque symétriques, à soies convergeant vers l'aire pédiale qui est glabre, plus fines et plus courtes à la périphérie.

Mandibules (Fig. 15Y-Z ). Pas d'aire stridulatoire. Aire scissoriale de la mandibule gauche allongée, avec 3 dents très émoussées, l'apicale bien développée, les deux autres arrondies, peu distinctes. Aire scissoriale de la mandibule droite allongée, avec deux dents émoussées, l'apicale développée et la seconde très effacée. 5 à 6 soies latéro-externes.

Maxilles (Fig. 17G). Galea et lacinia soudées à la base et séparées à l'apex. Lacinia à uncus avec 3 fortes dents sclérifiées et 4 soies épineuses sclérifiées. Galea à uncus sclérifié avec 6 très fortes et courtes soies épineuses sclérifiées sur la face inférieure et 6 fortes soies sclérifiées de l'apex à la face supérieure. Sur la face dorsale, aire stridulatoire avec 15 à 16 dents séparées, pointues, inclinées vers l'apex comme les dents d'une scie.

Hypopharynx (Fig. 12D). Transverse. Glosses recouvertes de soies longues et courtes mêlées, plus longues à l'apex et plus denses et plus fortes à la base. Oncyli transverses, avec 2 fortes dents sclérifiées tranchantes, l'une à plat, l'autre dressée.

\section{Thorax}

Pattes (Fig. 20E). Longues, de longueur croissante de P1 à P3, de structure analogue: coxa, trochanter inséré latéralement sur la coxa, fémur, tibiotarse. $\mathrm{P} 1$ et $\mathrm{P} 2$ à griffe longue, sublinéaire, large à la base, pointue, sclérifiée à l'apex, la griffe 1 plus longue que la 2. P3 à griffe courte, plus large que longue, à apex pointu sclérifié, portant 2 longues soies.

\section{Abdomen}

Raster (Fig. 5E). Fente anale en "Y». Palidia en forme d'ogive "gothique» dont la pointe serait tronquée, formés de longues et fortes soies densément réparties sur quatre rangs et orientées vers le septula, qui est glabre. Teges avec des soies aplaties, recourbées à l'apex, espacées, dessinant un large $\mathrm{V}$ renversé. Du côté dorsal de la fente anale, de très courtes soies coniques mêlées à des soies plus longues.

Famille ORPHNIDAE Erichson, 1847

Triodontus nitidulus (Guérin, 1844)

(Figs 2O; 5G; 9E; 11B; 12E; $13 \mathrm{E} ; 16 \mathrm{~A}-\mathrm{D} ; 17 \mathrm{H} ; 20 \mathrm{~F})$

\section{MATÉRIEL EXAMINÉ}

Larves provenant d'Andranomanelatra et d'Ambohimiarivo ( $8 \mathrm{~km}$ d'Andranomanelatra). Elles ont été collectées respectivement en haut de pente dans une culture de Riz pluvial (Oryza sativa) et dans une jachère à Cynodon dactylon et Brachiaria ruziziensis. En culture du Riz pluvial, les larves coupent les racines des plants. Type de sol: ferrallitique. Spécimens conservés dans les collections du Fofifa (22 larves), du Cirad-CBGP (4 larves) et du MNHN (2 larves). 
DESCRIPTION

Voir Paulian \& Lumaret (1982). Couleur blanche (Fig. 2O).

\section{Tête}

Couleur jaune. Épicrâne symétriquement avec 3 soies dorso-épicraniales et 5 soies latéro-externes. 1 soie de chaque côté de l'angle frontal. Clypéus avec 1 soie clypéale externe et 1 soie clypéale médiane.

Antennes. Articles effilés. Dernier article antennaire fusiforme avec 2 plages sensorielles: 1 ventrale et 1 dorsale.

Labre (Fig. 13E). Transverse, subsymétrique, trilobé, en forme générale de losange tronqué en arrière. Lobe médian étroit, formant une pointe anguleuse, dessinant la silhouette d'une pyramide à degrés, avec 3 groupes symétriques de 2 soies. $2+1$ longue soies latéro-antérieures. Soies de l'acanthoparia visibles latéralement, sur l'aire latéro-antérieure. 1 longue soie latérale. 2 longues soies labro-internes. 2 longues soies postérieures.

Épipharynx (Fig. 9E). Haptomère formé de 4 longues et fortes soies. Acanthoparia avec 9 courtes soies de taille croissante, incurvées vers l'avant. Chaetoparia subsymétriques, avec des rangées concentriques de soies épaisses convergeant vers l'aire pédiale, qui est glabre. Plegmatum développé, sa plus grande largeur le long des côtés antérieurs du losange. Acroparia avec 4 longues et fortes soies et 1 plus courte à droite et 4 longues soies à gauche. Corypha avec 4 longues et fortes soies.

Mandibules (Fig. 16A-D). Pas d'aire stridulatoire. Aire scissoriale de la mandibule gauche falciforme et étroite, avec 4 dents très émoussées, la dent apicale forte, suivie par 3 dents arrondies peu saillantes, la médiane plus grande. Aire scissoriale de la mandibule droite falciforme et étroite, la dent apicale forte et la seconde dent arrondie. Aire molaire de la mandibule gauche avec une forte dent bifide. 1 soie dorso-externe et 2 soies baso-latérales.

Maxilles (Fig. 17H). Galea et lacinia séparées. Lacinia à uncus avec 3 dents dont 2 fusionnées à la base.
Galea avec uncus acuminé. Sur la face dorsale, aire stridulatoire avec 15 dents inclinées et pointues.

Hypopharynx (Fig. 12E). Allongé, les côtés subparallèles légèrement concaves, palpes labiaux fins et longs. Glosses avec deux rangées de très fortes soies à la base, de longues soies dans la partie apicale, quelques courtes soies dispersées, glabres dans la partie médiane.

\section{Thorax}

Pattes (Fig. 20F). Longues, de longueur légèrement croissante de P1 à P3, de structure analogue: coxa, trochanter inséré latéralement sur la coxa, fémur, tibiotarse. Griffe longue, aiguë, incurvée, sclérifiée.

\section{Abdomen}

Raster (Fig. 5G). Fente anale en «Y». Palidia formés de 20 épines convergeant vers la fente anale, formant un arc de cercle, les plus longues vers l'anus, les plus courtes vers l'avant. Teges avec des soies épineuses. Côté dorsal de la fente anale glabre. Barbula avec de très longues soies.

\section{DIAGNOSES DES NYMPHES}

Les échantillons sont conservés dans les collections du MNHN, du Cirad (CBGP) et du Fofifa. Nous proposons le terme de «structures ano-génitales externes» (en anglais: «external genitoanal structures») pour désigner la partie préapicale de la face ventrale de l'abdomen, souvent caractérisée par un fort dimorphisme sexuel (Figs 11C, D; 21K, O). Cette notion est plus générale que celle de «developing genital capsule» (Edmonds \& Halffter 1978), cette capsule saillante ne désignant qu'une partie de cette structure et n'étant pas toujours présente. L'apex abdominal forme une double pointe plus ou moins obtuse ou effilée.

Famille CETONIIDAE Leach, 1815

Bricoptis variolosa (Gory \& Percheron, 1833) (Fig. 21A-C)

Couleur jaunâtre. Pronotum latéralement convexe et anguleux, deux fois plus large que long. Écusson 
triangulaire bien développé. Bords latéraux de l'abdomen légèrement convexes. Dernier segment abdominal à bords arrondis. 8 paires de stigmates abdominaux saillants de taille décroissante de la base vers l'apex, la première paire cachée sous les ptérothèques. Spécimens conservés dans les collections du Cirad-CBGP (1 nymphe) et du MNHN (1 nymphe).

\section{Euryomia argentea (Olivier, 1789)} (Fig. 21D-F)

Couleur jaunâtre. Pronotum deux fois plus large que long. Thorax plus large que l'abdomen. Pronotum latéralement concave. Abdomen effilé, à bords latéraux légèrement concaves. Dernier segment abdominal à bords arrondis. 8 paires de stigmates abdominaux saillants, la première paire cachée sous les ptérothèques. Spécimens conservés dans les collections du Fofifa (3 nymphes), du Cirad-CBGP (1 nymphe) et du MNHN (1 nymphe).

Famille Dynastidae MacLeay, 1819

Heteronychus arator rugifrons (Fairmaire, 1871) (Fig. 21G-I)

Couleur jaunâtre. Pronotum régulièrement arrondi, deux fois plus large que long. Thorax aussi large que l'abdomen. 4 paires de stigmates abdominaux ovales à bords sclérifiés, la première paire cachée dans une cavité sous les ptérothèques. 5 paires de lèvres incurvées sclérifiées, la première plus petite que les suivantes, formant 10 cavités disposées de part et d'autre de la ligne médiane, situées entre les tergites visibles I/II, II/III, III/ IV, IV/V et V/VI. Dernier segment abdominal à apex bifide émoussé, les deux lobes se touchant, chaque lobe couvert d'une touffe dense de poils dressés. Taille plus petite que celle de l'espèce suivante $(10,8$ à $17 \mathrm{~mm})$. Spécimens conservés dans les collections du Fofifa (3 nymphes), du Cirad-CBGP (2 nymphes) et du MNHN (1 nymphe).

\section{Heteronychus plebeius (Klug, 1833)}

(Fig. 21J-Q)

Couleur jaunâtre. Pronotum arrondi, deux fois plus large que long. Thorax aussi large que l'abdomen. 4 paires de stigmates abdominaux ovales à bords sclérifiés, la première paire cachée dans une cavité sous les ptérothèques. 5 paires de lèvres incurvées sclérifiées, la première plus petite que les suivantes, formant 10 cavités disposées de part et d'autre de la ligne médiane, situées entre les tergites visibles I/II, II/ III, III/IV, IV/V et V/VI. Dernier segment abdominal à apex bifide aigu, les deux lobes espacés, chaque lobe couvert d'une touffe dense de poils dressés. Taille plus grande que celle de l'espèce précédente (14,6 à $21 \mathrm{~mm})$. Spécimens conservés dans les collections du Fofifa (2 nymphes), du Cirad-CBGP (2 nymphes) et du MNHN (1 nymphe).

\section{Dimorphisme sexuel}

Mâle (Fig. 21J-M). Silhouette plus large, l'abdomen plus arrondi. Tarses antérieurs plus larges. Cavités des tergites plus larges. Structures ano-génitales externes quadrilobées vers l'arrière.

Femelle (Fig. 21N-Q). Silhouette plus étroite, l'abdomen plus effilé. Tarses antérieurs plus étroits. Cavités des tergites plus étroites. Structures anogénitales externes unilobées vers l'arrière.

\section{Hexodon unicolor (Olivier, 1789)}

(Fig. 21R-T)

Couleur jaunâtre. Pronotum en forme de croissant de lune, deux fois plus large que long, les bords latéraux nettement saillants et se terminant en une pointe émoussée. Abdomen fusiforme. Première paire de stigmates abdominaux cachée dans une cavité sous les ptérothèques et le bord latéral du premier tergite visible; 3 paires de stigmates saillants à bords sclérifiés sur les tergites II-IV; 4 paires de stigmates vestigiaux sur les tergites V-VIII. 5 paires de lèvres incurvées sclérifiées, la dernière plus petite que les précédentes, formant 10 cavités disposées de part et d'autre de la ligne médiane, situées entre les tergites visibles I/II, II/III, III/IV, IV/V et V/VI. 
Spécimens conservés dans les collections du Fofifa (1 nymphe) et du MNHN (1 nymphe).

Heteroconus paradoxus (Endrödi, 1968) (Fig. 21U-W)

Couleur jaune-brunâtre. Pronotum régulièrement arrondi, deux fois plus large que long. Thorax aussi large que l'abdomen. 4 paires de stigmates abdominaux circulaires à bords sclérifiés, la première paire cachée dans une cavité sous les ptérothèques et un repli latéral du premier tergite visible. 5 paires de lèvres incurvées sclérifiées de taille croissante d'avant en arrière, formant 10 cavités disposées de part et d'autre de la ligne médiane, situées entre les tergites visibles I/II, II/III, III/IV, IV/V et V/VI. Spécimens conservés dans les collections du Fofifa (7 nymphes), du Cirad-CBGP (2 nymphes) et du MNHN (1 nymphe).

Paranodon coquerelii (Fairmaire, 1871) (Fig. 22A-C)

Couleur jaune-brunâtre. Pronotum de forme arrondie, deux fois plus large que long, dépassant légèrement le thorax. Thorax aussi large que l'abdomen. 4 paires de stigmates abdominaux ovales à bords sclérifiés, la première paire de plus petite taille, cachée dans une cavité sous les ptérothèques et un repli latéral du premier tergite visible. 5 paires de lèvres incurvées sclérifiés formant 10 cavités disposées de part et d'autre de la ligne médiane, situées entre les tergites visibles I/II, II/III, III/IV, IV/V et V/VI, la première paire moins large que les suivantes et avec un épaississement sclérifié vers l'avant. 2 petits lobes hémisphériques accolés en face dorsale de l'apex abdominal. Spécimens conservés dans les collections du Fofifa (1 nymphe) et du Cirad-CBGP (1 nymphe).

Famille Hoplinde Latreille, 1829

Paramorphochelus cornutus (Nonfried, 1892) (Fig. 22D, E)

Couleur jaunâtre. Silhouette trapue, les côtés sub- parallèles. Pronotum bien plus large que long, à bords latéraux anguleux saillants vers l'avant. Pattes postérieures très longues, repliées au niveau de l'apex abdominal. Première paire de stigmates abdominaux ronds et de petite taille cachés sous les ptérothèques; 3 paires de stigmates en relief sur les tergites II-IV; 3 paires de stigmates vestigiaux sous forme de micro-reliefs sur les tergites V-VII; 1 paire de stigmates vestigiaux formant un relief plus saillant que les 3 paires précédentes sur le tergite VIII. Fortes épines sclérifiées incurvées vers l'arrière sur la ligne médiane des tergites abdominaux visibles II à VI. Apex abdominal avec 2 crochets pointus et sclérifiés, très écartés et incurvés vers l'intérieur. Spécimens conservés dans les collections du Cirad (1 nymphe) et du Fofifa (2 nymphes).

Famille Melolonthidae MacLeay, 1819

Encya sikorai (Brenske, 1891)

(Figs $11 \mathrm{C}, \mathrm{D} ; 22 \mathrm{~F}-\mathrm{K}$ )

Couleur jaune nuancée de brun-rougeâtre. Pronotum nettement plus large que long. Première paire de stigmates abdominaux cachée sous les ptérothèques et le bord latéral du premier tergite visible; les trois paires suivantes de forte taille et très sclérifiées sur les tergites II-IV, 2 paires de stigmates effacés sur les tergites V-VI; 2 paires de stigmates vestigiaux sur les tergites VII-VIII. Spécimens conservés dans les collections du Fofifa (1 nymphe) et du Cirad (2 nymphes).

\section{Dimorphisme sexuel}

Très fortement marqué, le dimorphisme sexuel préfigure celui des imagos: "Chez la femelle, les tarses postérieurs sont plus courts [...]; la forme est plus massive» (Lacroix 1993: 349).

Mâle (Figs 11C; 22F-H). Silhouette moins massive. Yeux moins gros et moins saillants. Tarses postérieurs très longs, divergents, saillants sur les côtés. Métathorax régulièrement arrondi dorsalement. Segments abdominaux VII et suivants bien plus étroits que les précédents. Apex abdominal lisse sur la majeure partie de la surface, très partiellement 
ridé, structures ano-génitales externes formant des reliefs longitudinaux peu saillants. Crochets apicaux effilés, allongés, légèrement écartés, divergents en "V», sclérifiés. (Fig. 11C).

Femelle (Figs 11D; 22I-K). Silhouette plus massive. Yeux plus gros et plus saillants. Tarses postérieurs de longueur normale, convergents. Métathorax avec dorsalement deux forts reliefs arrondis. Abdomen plus large, devenant progressivement plus étroit vers l'apex. Apex abdominal entièrement ridé, structures ano-génitales externes formant une fente transversale entourée d'un fort bourrelet précédée par une ampoule arrondie fortement saillante. Crochets apicaux massifs, divergeant en un « $U$ » très évasé formant presque un «T», terminés chacun par une paire de très courtes épines sclérifiées inégales. (Fig. 11D).

Enaria melanictera (Klug, 1833) (Fig. 22L-N)

Couleur jaune-ivoire très pâle. Silhouette étroite, fusiforme. Pronotum fortement transverse, à bord antérieur subrectiligne, arrondi sur les côtés. Une rangée transversale de courtes soies espacées sur les quatre premiers sternites visibles. Première paire de stigmates abdominaux de grande taille, ovale, cachée sous les ptérothèques; 3 paires de stigmates circulaires en relief de plus petite taille sur les tergites II-IV; 4 paires de stigmates vestigiaux sur les tergites V-VIII. Apex abdominal très effilé, avec une paire de crochets apicaux portant chacun deux courtes épines non sclérifiées et de taille inégale à l'apex. Spécimens conservés dans les collections du Fofifa (2 nymphes), du Cirad-CBGP (1 nymphe) et du MNHN (1 nymphe).

\section{Apicencya waterloti (Dewailly, 1950)}

(Fig. 22O-Q)

Couleur jaunâtre. Silhouette fusiforme, très large, effilée aux extrémités. Pronotum subtriangulaire. Épines orientées vers l'arrière sur les tergites 2 à 5 . Épines espacées sur les quatre premiers sternites visibles. Première paire de stigmates abdominaux cachée sous les ptérothèques; 3 paires de stigmates bien développés sur les tergites II-IV; 2 paires de stigmates effacés sur les tergites $\mathrm{V}-\mathrm{VI} ; 2$ paires de stigmates vestigiaux sur les tergites VII-VIII. Structures ano-génitales externes avec une capsule génitale saillante de forte taille. Crochets apicaux larges, incurvés, chacun portant 2 courtes épines à l'apex, légèrement écartés, convergents, non sclérifiés. Spécimens conservés dans les collections du Fofifa (1 nymphe) et du Cirad-CBGP (1 nymphe).

Empecta scutata (Fairmaire, 1901)

(Fig. 22R-T)

Couleur jaunâtre. Silhouette fusiforme et large. Pronotum plus ou moins arrondi. Segments abdominaux visibles VII à X un peu plus étroits que les précédents. Crochets apicaux pointus, fins, avec un petit lobe arrondi à la base, accolés, divergents en «T». Première paire de stigmates abdominaux cachée sous les ptérothèques, aux contours émoussés, plus petits que ceux des tergites suivants; 1 paire de stigmates aux contours bien marqués sur les tergites II-IV; 1 paire de stigmates effacés sur les tergites V-VI; 1 paire de stigmates vestigiaux sur les tergites VII-IX. Spécimens conservés dans les collections du Fofifa (4 nymphes) et du MNHN (1 nymphe).

\section{Hoplochelus betanimena (Künckel, 1887)}

(Fig. 22U-W)

Couleur jaunâtre. Silhouette fusiforme et étroite. Pronotum antérieurement anguleux. Première paire de stigmates abdominaux cachée sous les ptérothèques, plus petits que ceux des tergites suivants; 1 paire de stigmates aux contours bien marqués et sclérifiés sur les tergites II-IV; 1 paire de stigmates effacés sur les tergites V-IX. Segments abdominaux visibles VII à X un peu plus effilés que les précédents. Crochets apicaux petits, effilés, accolés, divergents en "V», entièrement sclérifiés. Spécimens conservés dans la collection du Fofifa (1 nymphe) et du MNHN (1 nymphe). 


\section{Clé D'IDENTIFICATION DES «VERS BLANCS» (SCARABAEOIDEA) PRÉSENTS EN RIZICULTURE PLUVIALE D'ALTITUDE À MADAGASCAR}

Le nombre de dents stridulatoires maxillaires, comme le nombre d'épines des palidia, sont mentionnés à titre indicatif, car ils sont sujets à une certaine variabilité.

1. Maxilles à galea et lacinia distinctement séparées (Fig. 17H). Fente anale en «Y». Palidia formant un cercle ouvert vers l'arrière d'épines convergeant vers la fente anale (Fig. 5G)

Orphnidae: Triodontus nitidulus

- Maxilles à galea et lacinia au moins partiellement soudées ou étroitement accolées l'une à l'autre. Fente anale transverse ou en «Y» 2

2. Fente anale transverse, droite ou faiblement arquée. Mandibules à aire stridulatoire ventrale formant une plage ovale, avec un nombre variable de stries transversales (Fig. 13J), réduite à 5 stries difficilement visibles chez Heteroconus paradoxus. Galea et lacinia soudées sur toute leur longueur, la suture plus ou moins visible ou effacée .................................. 3

— Fente anale en «Y». Mandibules sans aire stridulatoire ventrale .................................... 8

3. Palidia en général présents, parfois absents. Lacinia avec un uncus formé de 2 dents sclérifiées. Dents stridulatoires maxillaires constituées de fortes pointes sclérifiées subconiques et pointues. Labre bilobé ou trilobé, en général presque symétrique, avec une tache pigmentée de part et d'autre du milieu, sur le bord antérieur (clithra) (Fig. 13A). Haptomère avec en général une rangée transverse arquée de courtes et fortes soies épineuses, jamais conformé en pointe saillante (Figs 6G, H; 7A, B). Larves se déplaçant sur le dos .... Cetoniidae. 4

- Palidia toujours absents. Lacinia avec uncus formé de 2 ou 3, parfois 4 dents sclérifiées. Labre asymétrique (Fig. 13B). Haptomère en pointe saillante (Figs 7C-H; 8A, B; 11A). Larves se déplaçant sur le ventre ........................................................ Dynastidae. 18

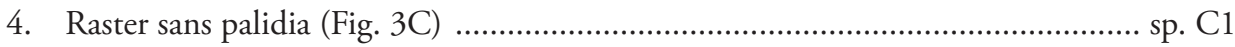

— Raster avec palidia (Fig. 3A, B, D, E) ……........................................................ 5

5. Palidia (Fig. 3A) formés par 2 rangées longitudinales de 12 à 19 épines légèrement concaves. $5+1$ dents stridulatoires maxillaires (Fig. 16E) Bricoptis variolosa

- Palidia conformés autrement, soit avec 2 rangées d'épines divergentes vers l'arrière (Fig. 3B), soit avec 2 rangées d'épines subparallèles (Fig. 3D), soit avec 2 rangées d'épines légèrement convexes (Fig. 3E) .. 6

6. Palidia (Fig. 3B) formés par 2 rangées longitudinales de 16 à 21 épines divergeant à l'extrémité postérieure, avec une rupture de la courbe au tiers basal environ (formant ainsi le dessin d'un «fer de lance»). $5+1$ dents stridulatoires maxillaires (Fig. 16F) Celidota parvula

- Palidia formés d'alignements de 11 à 16 épines disposées soit en 2 rangées subparallèles (Fig. 3D), soit en 2 rangées légèrement convexes dessinant une "voûte gothique» vers l'avant (Fig. 3E) 7

7. Palidia (Fig. 3E) formés par deux rangées légèrement convexes de 13 à 16 épines, formant une pointe antérieure en «ogive gothique». $4+1$ dents stridulatoires maxillaires (Fig. 16H)

Euryomia argentea

- Palidia (Fig. 3D) formés par 2 rangées subparallèles de 11 à 14 épines. 6 à $8+1$ dents stridulatoires maxillaires (Fig. 16G) Anochilia bifida

8. Palidia absents, raster avec un groupe de fortes soies aplaties et recourbées à l'apex du côté ventral de la fente anale, avec de nombreuses soies plus fines autour de la fente anale et du côté dorsal de celle-ci (Fig. 4F) Hopliidae: Paramorphochelus cornutus 
9. Palidia formés par 2 rangées ou groupes symétriques d'épines séparés par le septula glabre (Figs 4G, H; 5A, C-F). Si le palidium est formé par une rangée d'épines disposées en arc de cercle, alors celles-ci sont regroupées en "touffes» de 2, 3 ou 4 soies rapprochées (Fig. 5B). Galea et lacinia fusionnées à la base mais séparées et accolées à l'apex

Melolonthidae. 10

- Palidium formé par une rangée d'épines régulièrement disposées en arc de cercle ouvert vers l'arrière (Fig. 5H, I) Sericidae. 17

10. Soit palidium formé par une rangée de fortes soies disposées en arc de cercle et regroupées en «touffes» de 2, 3 ou 4 soies rapprochées (Fig. 5B), soit palidia formés par une double rangée symétrique de soies dessinant un $\mathrm{V}$ renversé (Fig. 5A) ou un fuseau (Fig. 5C)

- Palidia formés non par une double rangée symétrique de soies mais soit par un double groupe symétrique de soies (Figs 4G, H; 5E, F), soit par une double rangée symétrique de courtes épines en avant et de 2 groupes symétriques de fortes et longues soies en arrière, l'ensemble formant un «T» inversé (Fig. 5D) 11

11. Palidia (Fig. 5D) formés par une double rangée symétrique de courtes épines en avant et par 2 groupes symétriques de fortes et longues soies en arrière, l'ensemble formant un "T» inversé. 13 à 14 dents stridulatoires maxillaires (Fig. 17F) Empecta scutata

— Palidia formés par 2 groupes symétriques de fortes soies (Figs 4G, H; 5E, F) ........... 12

12. Palidia (Fig. 4G) formés par 2 groupes symétriques de fortes soies en forme de "J»

- Palidia (Figs 4H; 5E, F) formés par 2 groupes subtriangulaires symétriques de fortes soies 13

13. Palidia (Fig. 5F) formés par 2 groupes de fortes soies disposées sur 2 ou 3 rangs au maximum sp. M3

- Palidia (Figs 4H; 5E) formés par 2 groupes de fortes soies plus nombreuses disposées sur 4 rangs ou plus 14

14. Palidia (Fig. 4H) en triangle plus large que haut. Teges plus étroits, aux soies plus courtes disposées sur 3 rangs. 17 à 21 dents stridulatoires maxillaires (Fig. 17C) .... Encya sikorai

- Palidia (Fig. 5E) en triangle plus haut que large, en "ogive gothique» dont la pointe serait tronquée. Teges plus large, jusqu'à 5 rangs de soies plus allongées. 15 à 16 dents stridulatoires maxillaires (Fig. 17G) Hoplochelus betanimena

15. Palidia (Fig. 5A) en forme de "V» renversé. 11 à 13 dents stridulatoires maxillaires (Fig. 17D) Enaria melanictera

- Palidia (Fig. 5B, C) arrondis 16

16. Palidium (Fig. 5B) formé par une rangée de fortes soies disposées en arc de cercle et regroupées en "touffes" de 2,3 ou 4 soies rapprochées sp. M2

- Palidia (Fig. 5C) formés par une double rangée symétrique de 13-15 fortes soies dessinant un fuseau. 16 à 17 dents stridulatoires maxillaires (Fig. 17E) Apicencya waterloti

17. Palidia (Fig. 5H) formés par 15 à 18 longues épines aplaties très serrées, pointues

- Palidia (Fig. 5I) formés par 17 à 19 courtes épines aplaties, plus espacées, à apex arrondi sp. S2 
18. Raster (Fig. 4E) avec symétriquement de part et d'autre du milieu, séparées par une étroite zone glabre, une très forte et épaisse épine variable orientée vers l'intérieur (simple, bifide ou trifide) et dissymétrique (trifide ou bifide du côté gauche et bifide ou simple du côté droit). 9 à 10 dents stridulatoires maxillaires (Fig. 17A) ................ Paranodon coquerelii

— Raster sans fortes soies bifides ou trifides 19

19. Raster (Fig. 3F) avec une large zone médiane glabre centrale bordée d'une rangée de 5 ou 6 courtes épines du côté dorsal de la fente anale. 7 à $10+1$ dents stridulatoires maxillaires (Fig. 16J) Heteronychus arator rugifrons

- Raster sans large zone médiane glabre centrale du côté dorsal de la fente anale 20

20. Raster (Fig. 4C) : teges à soies épineuses courtes non recourbées à l'apex. Larve L3 toujours de très grande taille. sp. D2

- Raster: teges à soies épineuses aplaties recourbées à l'apex. Larve L3 de taille petite à grande

21. Raster (Fig. 4A): teges avec des épines aplaties et recourbées assez étroites, très nombreuses et avec de longues soies sur la périphérie. Larve L3 toujours de grande taille ...... sp. D1

- Raster (Figs 3G, H; 4B, D) : teges jamais entourés par de longues soies, avec des épines aplaties et recourbées assez larges, moins nombreuses. Larve L3 de taille petite à grande

22. Raster (Fig. 4B) : teges avec de nombreuses soies aplaties et recourbées. Du côté dorsal de la fente anale, nombreuses soies courtes mêlées de quelques longues soies. Une étroite bande glabre le long de la fente anale du côté dorsal. 9 à 10 dents stridulatoires maxillaires (Fig. 16N). Larves L3 de grande taille Hexodon unicolor

- Raster conformé autrement (Figs 3G, H ; 4D), teges à épines moins nombreuses. Larves L3 de petite taille

23. Raster (Fig. 4D) : teges avec de très fortes soies dispersées, épaisses, recourbées et aplaties. Du côté dorsal de la fente anale, nombreuses soies courtes avec quelques très longues soies fines. Mandibules à aire stridulatoire réduite à 5 stries difficilement visibles. 12 à 15 dents stridulatoires maxillaires (Fig. 16O) Heteroconus paradoxus

- Raster conformé autrement (Fig. 3G, H) 24

24. Raster (Fig. 3G) : teges avec de fortes et longues soies aplaties, à l'apex incurvé, avec de chaque côté un groupe de soies plus longues. 5 dents stridulatoires maxillaires Heteronychus bituberculatus

- Raster (Fig. $3 \mathrm{H}$ ) : teges avec des soies bien plus courtes que chez $H$. bituberculatus, sans groupe de longues soies de chaque côté. $7+1$ dents stridulatoires maxillaires

Heteronychus plebeius

\section{DISCUSSION}

Dans le Centre de Madagascar, en riziculture pluviale de haute et de moyenne altitudes, plusieurs types de «Vers blancs» peuvent être trouvés à différentes profondeurs dans le même prélèvement de sol (Randriamanantsoa données non publiées) et nous avons recensé au total 26 espèces et morpho-espèces dans l'ensemble des localités prospectées. Presque tous les «Vers blancs» se ressemblent et la relative uniformité de leurs habitus impose de recourir à des caractères morphologiques fins pour séparer les espèces. Jusqu’à présent, dans ce contexte agronomique, aucune publication ne permettait d'identifier les taxons, qui demeuraient confondus, et il était impossible de distinguer les ravageurs majeurs, les ravageurs mineurs ou potentiels et les espèces potentiellement utiles, ce qui interdisait toute gestion raisonnée. Si le raster offre d'excellents 
caractères, suffisants dans un premier temps pour séparer les morpho-espèces et les identifier, il ne faut pas négliger pour autant les autres caractères.

Si le complexe «Vers blancs» a toujours été considéré comme ravageur important des cultures, il s'avère que certaines espèces qui le constituent n'ont pas d'incidence notable (Randriamanantsoa et al. 2008) ou même améliorent la structure du sol sur les Hauts Plateaux malgaches (Ratnadass et al. 2006). Dans d'autres publications, nous analysons l'impact de chaque espèce sur la culture et nous étudions la dynamique des populations des "Vers blancs» (Randriamanantsoa et al. 2008; Randriamanantsoa données non publiées).

Malgré des échantillonnages nombreux et répétés, certaines morpho-espèces très peu fréquentes n'ont été trouvées qu'une seule fois. Nous n'avons donc pas pu les élever ni les identifier, mais leur incidence dans le contexte agronomique des stations étudiées est négligeable. Par la réalisation de ce premier inventaire et par la mise au point de la diagnose des larves et d'une clé des espèces, nous avons défriché un terrain vierge et donné un outil de gestion à la riziculture pluviale des régions de haute et moyenne altitudes du Centre de Madagascar.

Dans le strict cadre de la présente étude, il reste encore à déterminer huit morpho-espèces. La nomenclature des Sericidae malgaches est entièrement à revoir (Lacroix comm. pers.) et aucune détermination fiable des imagos (que nous avons obtenus pour $\mathrm{S} 1$ et S2) n'est encore possible.

La connaissance de la biologie des espèces recensées n'est pas suffisante et mérite d'être approfondie, surtout dans le cas de celles qui ont une importance économique.

Quand nous avons identifié les espèces, des difficultés techniques rendaient impossible l'extraction d'ADN utilisable à partir des larves de Scarabaeoidea ou à partir d'imagos conservés à sec, ce qui n'est plus le cas aujourd'hui. Les futures recherches consacrées aux «Vers blancs» à Madagascar, sans abandonner les méthodes classiques, devront s'appuyer sur le barcoding pour contrôler la connexion larves-adultes, limitant ainsi les contraintes - et les risques d'erreur - de l'élevage.

La riziculture pluviale étant pratiquée dans presque toutes les régions de Madagascar, y compris à basse altitude, et étant donné la variabilité des écosystèmes (altitude, relief, exposition, climat, nature du sol...), on peut s'attendre à enrichir le recensement des espèces en prospectant ailleurs. C'est cependant dans les terres de haute et moyenne altitudes que le Riz pluvial est le plus cultivé et auxquelles le cadre de notre recherche est volontairement circonscrit. Les espèces de «Vers blancs» que nous avons collectées au cours de cette étude constituent donc un échantillonnage assez représentatif de la faune des rizières pluviales de cette zone géographique. Pour compléter cet inventaire, c'est dans la région du lac Alaotra, d'altitude moyenne (853 m), où l'attaque des «Vers blancs» est importante, qu'il faudrait en priorité recenser les espèces.

\section{Remerciements}

Nous remercions vivement les personnes et les institutions qui nous ont aidés: le Conseil régional de l'île de la Réunion, pour sa participation au financement de notre travail à travers la Convention MCR/2003/1155 signée entre Madagascar et la Réunion; la Direction et le personnel du Centre national de Recherches appliquées au Développement rural (Fofifa), pour nous avoir donné l'opportunité d'accomplir ce travail; le Cirad, à travers la Délégation aux Échanges scientifiques internationaux (Desi), pour l'accueil, l'organisation et le financement des séjours de R. Randriamanantsoa à Montpellier en 2004, 2005 et 2007; le Centre de Biologie pour la Gestion des Populations (CBGP) à Montpellier, qui a accueilli R. Randriamanantsoa dans ses locaux; MM. G. Delvare \& J.-Y. Rasplus, du CBGP, pour avoir accueilli R. Randriamanantsoa dans leur laboratoire; Mme L. Ollivier, du Cirad, pour la mise à notre disposition du matériel photo Leica; M. L. Soldati, pour la mise à notre disposition du matériel de prise de vue Entovision; MM. P. Renaud, J.-C. Streito, J.-F. Germain, M. Martinez, J.-M. Ramel, E. Pierre, V. Balmes \& C. Cocquempot du Laboratoire national de la Protection des Végétaux (LNPV) et de l'Inra à Montpellier pour leur accueil, leur collaboration et la mise à notre disposition du matériel photo; MM. M. Lacroix, P. Antoine et J.-B. Huchet, pour la détermination de certaines espèces et pour la documentation communiquée; M. R.-P. Dechambre, pour le prêt de spécimens de Dynastidae du MNHN; M. B. C. Ratcliffe, 

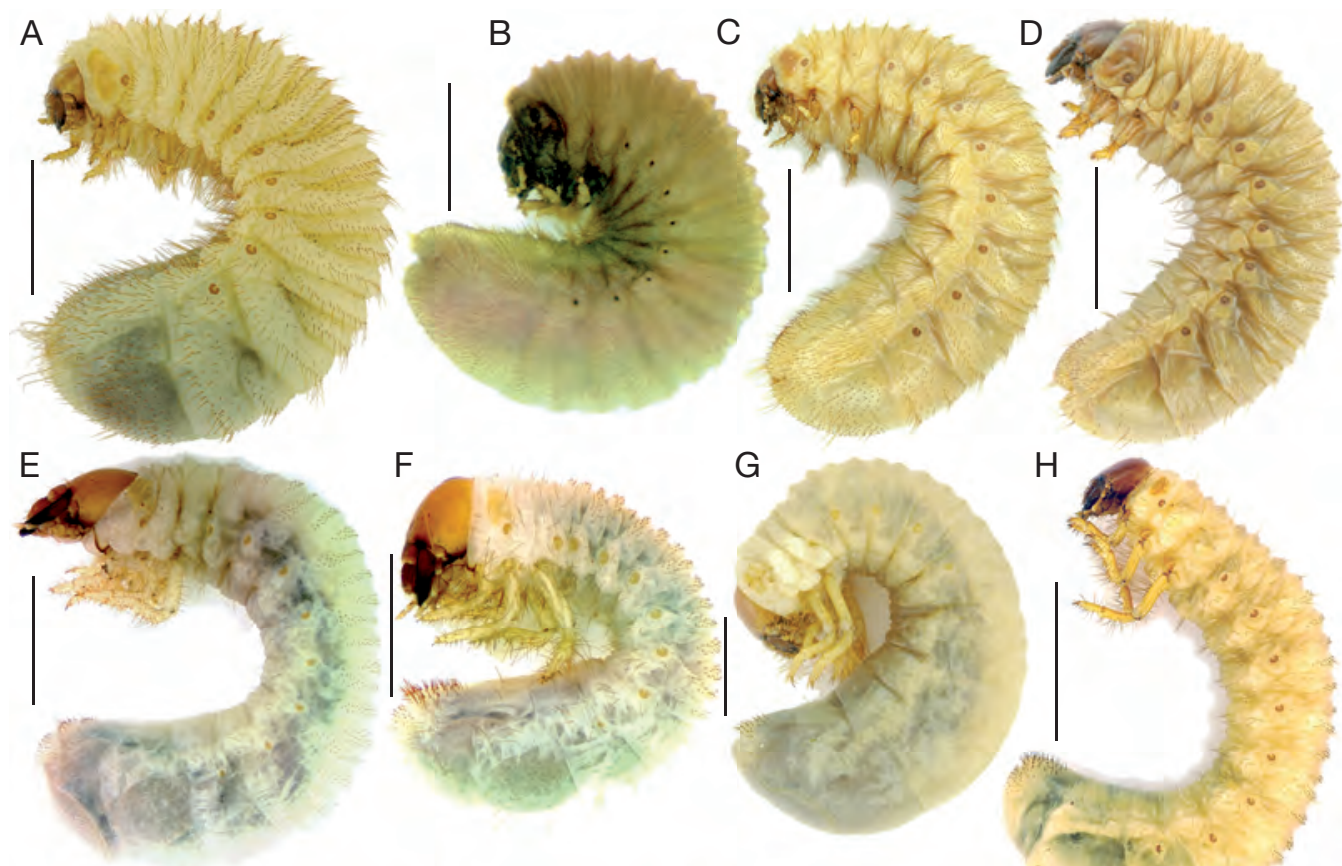

G
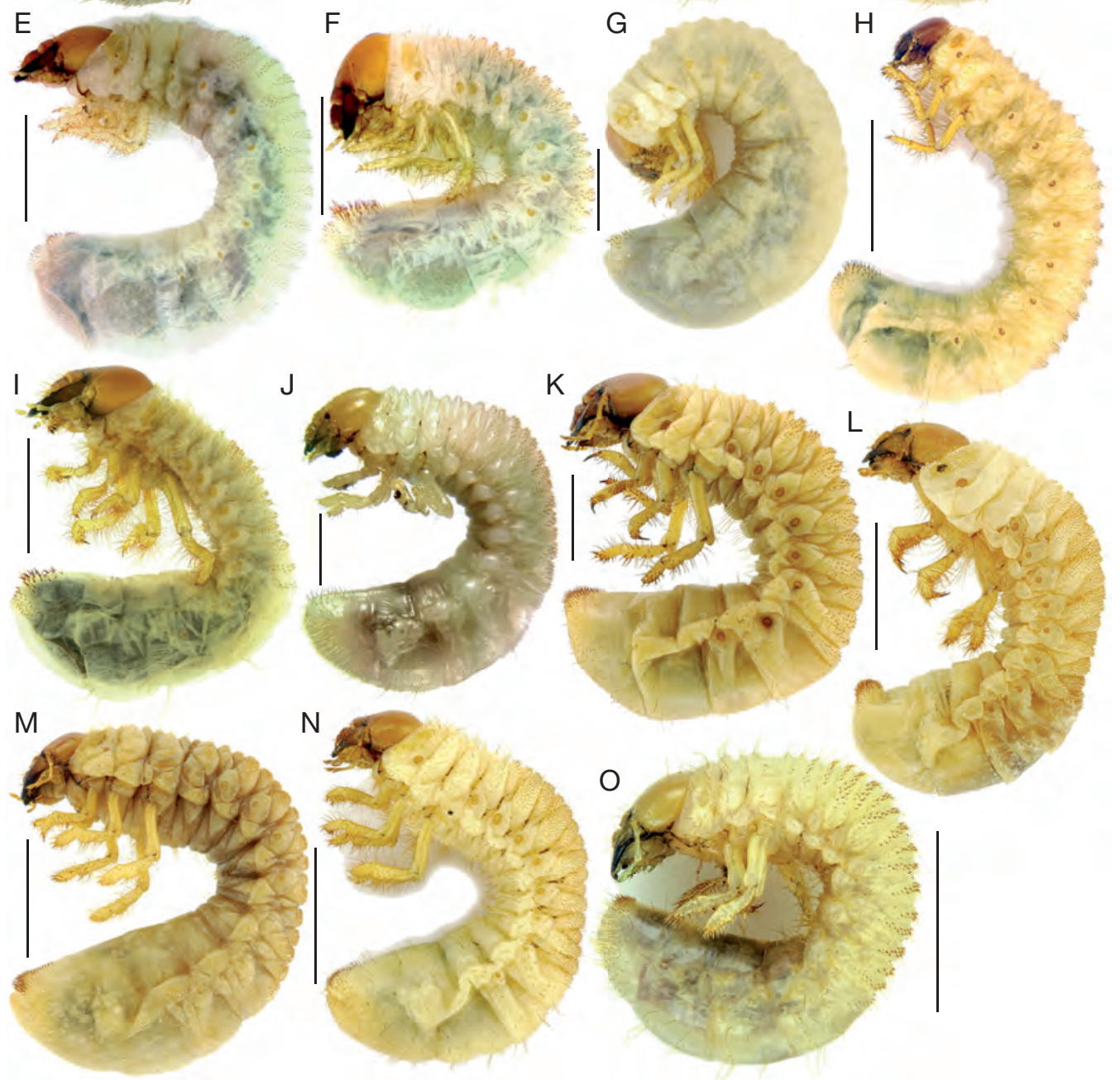

FIG. 2. - Larves de Scarabaeoidea de Madagascar, habitus: A, Bricoptis variolosa (Gory \& Percheron, 1833); B, Celidota parvula (Janson, 1881); C, Anochilia bifida (Olivier, 1789); D, Euryomia argentea (Olivier, 1789); E, Heteronychus arator rugifrons (Fairmaire, 1871); F, Heteronychus bituberculatus (Kolbe, 1900); G, Heteronychus plebeius (Klug, 1833); H, Hexodon unicolor (Olivier, 1789); I, Heteroconus paradoxus (Endrödi, 1968); J, Paramorphochelus cornutus (Nonfried, 1892); K, Encya sikorai (Brenske, 1891); L, Apicencya waterloti (Dewailly, 1950); M, Empecta scutata (Fairmaire, 1901); N, Hoplochelus betanimena (Künckel, 1887); O, Triodontus nitidulus (Guérin, 1844). Échelles: A-I, K, L, N, $5 \mathrm{~mm}$; J, O, $2 \mathrm{~mm}$; M, $3 \mathrm{~mm}$. 


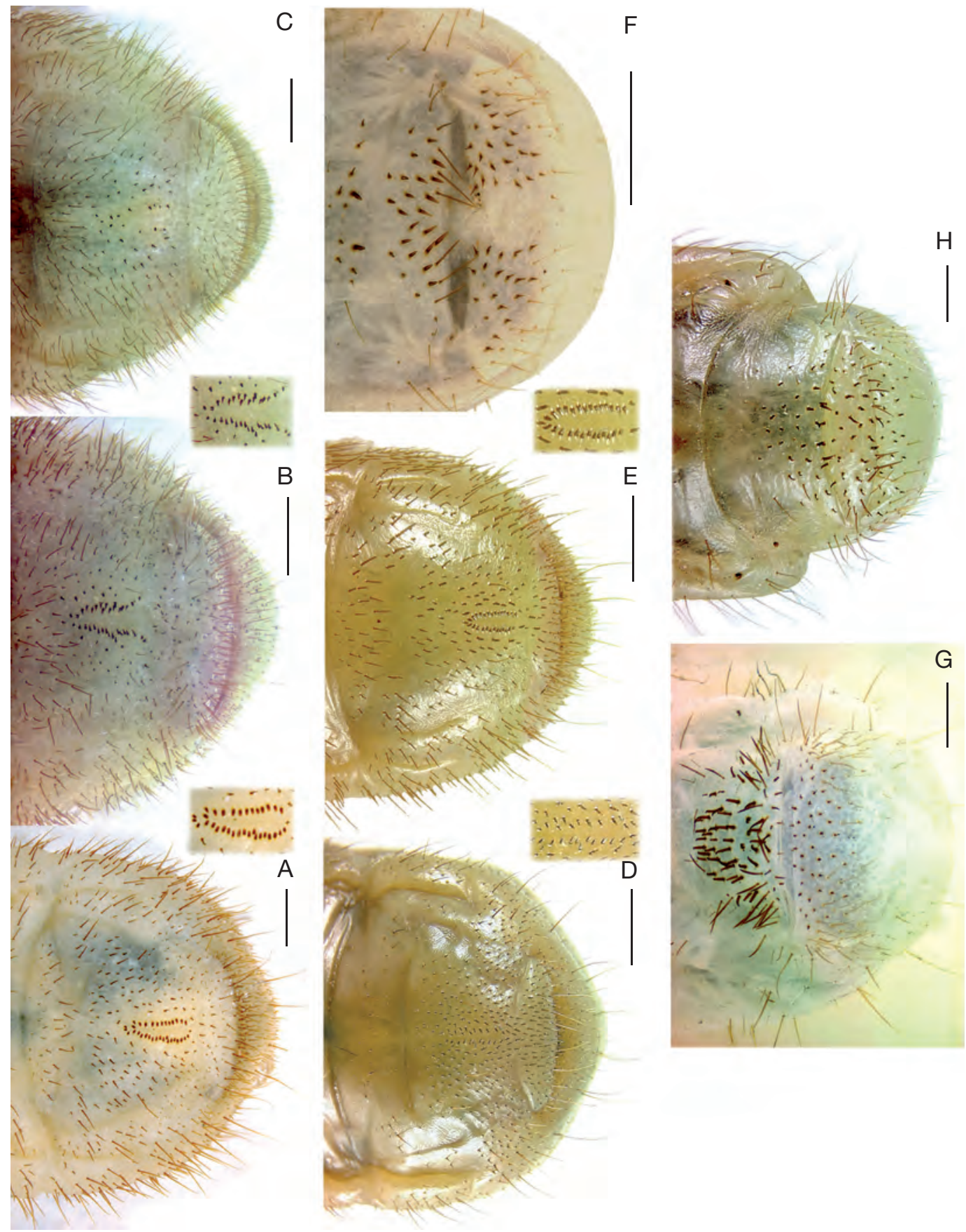

FIG. 3. - Larves de Scarabaeoidea de Madagascar, raster: A, Bricoptis variolosa (Gory \& Percheron, 1833); B, Celidota parvula (Janson, 1881); C, sp. C1; D, Anochilia bifida (Olivier, 1789); E, Euryomia argentea (Olivier, 1789); F, Heteronychus arator rugifrons (Fairmaire, 1871); G, Heteronychus bituberculatus (Kolbe, 1900); H, Heteronychus plebeius (Klug, 1833). Échelles: A-C, G, H, 1 mm; D, 0,5 mm; E, F, $2 \mathrm{~mm}$. 


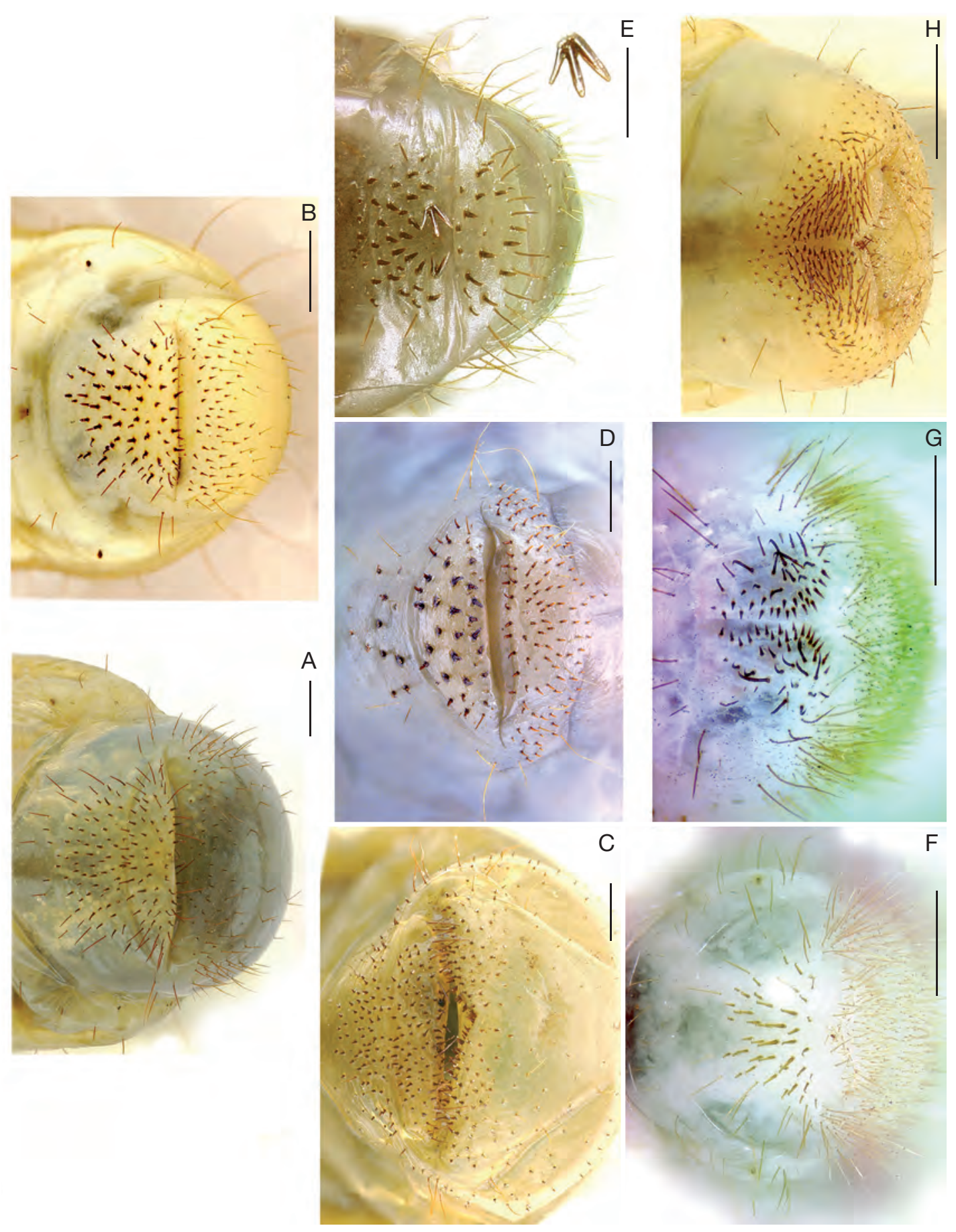

FIG. 4. - Larves de Scarabaeoidea de Madagascar, raster: A, sp. D1; B, Hexodon unicolor (Olivier, 1789); C, sp. D2; D, Heteroconus paradoxus (Endrödi, 1968); E, Paranodon coquerelii (Fairmaire, 1871); F, Paramorphochelus cornutus (Nonfried, 1892); G, sp. M1; H, Encya sikorai (Brenske, 1891). Échelles: A-D, H, 2 mm; E-G, 1 mm. 

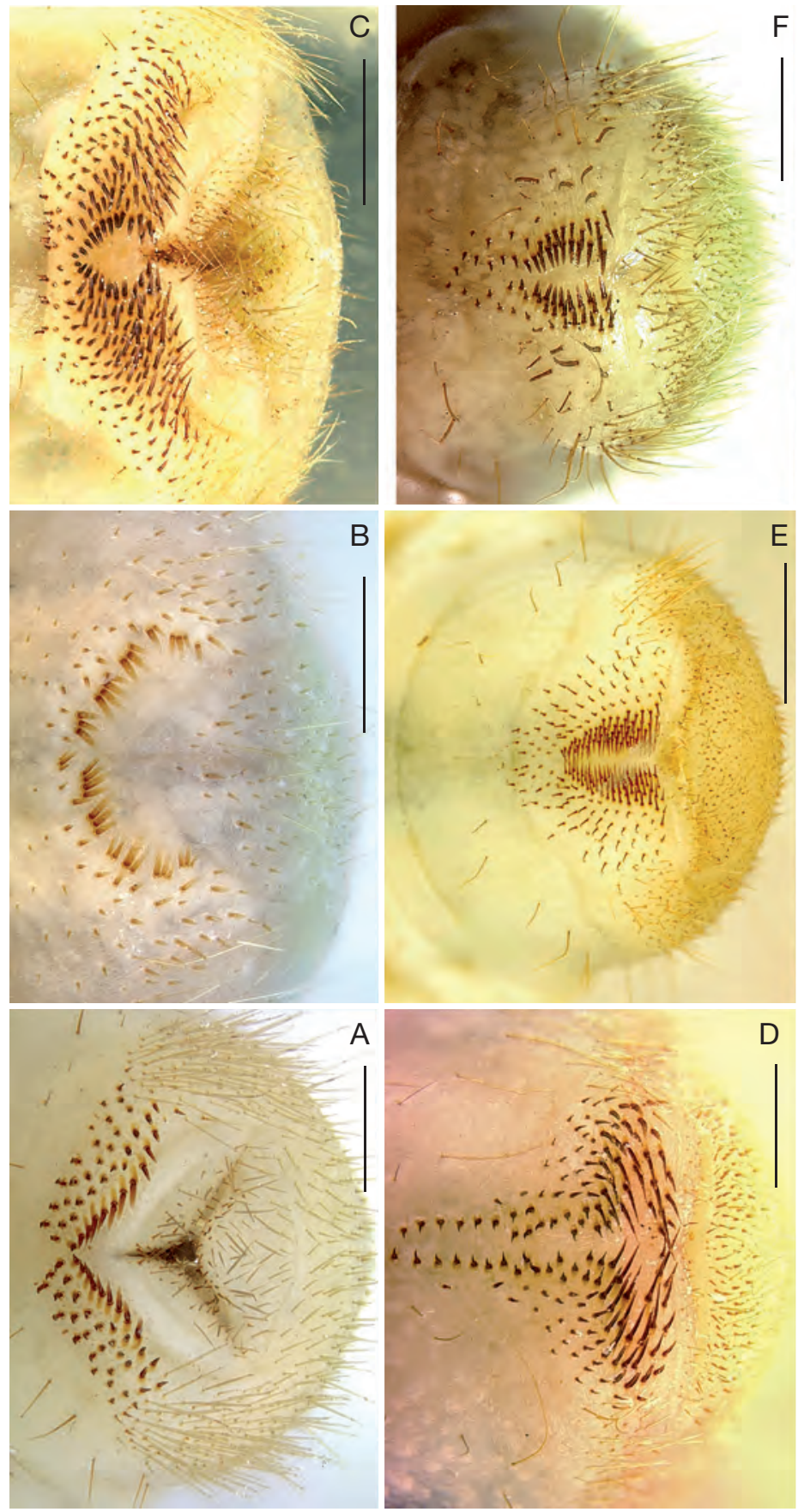
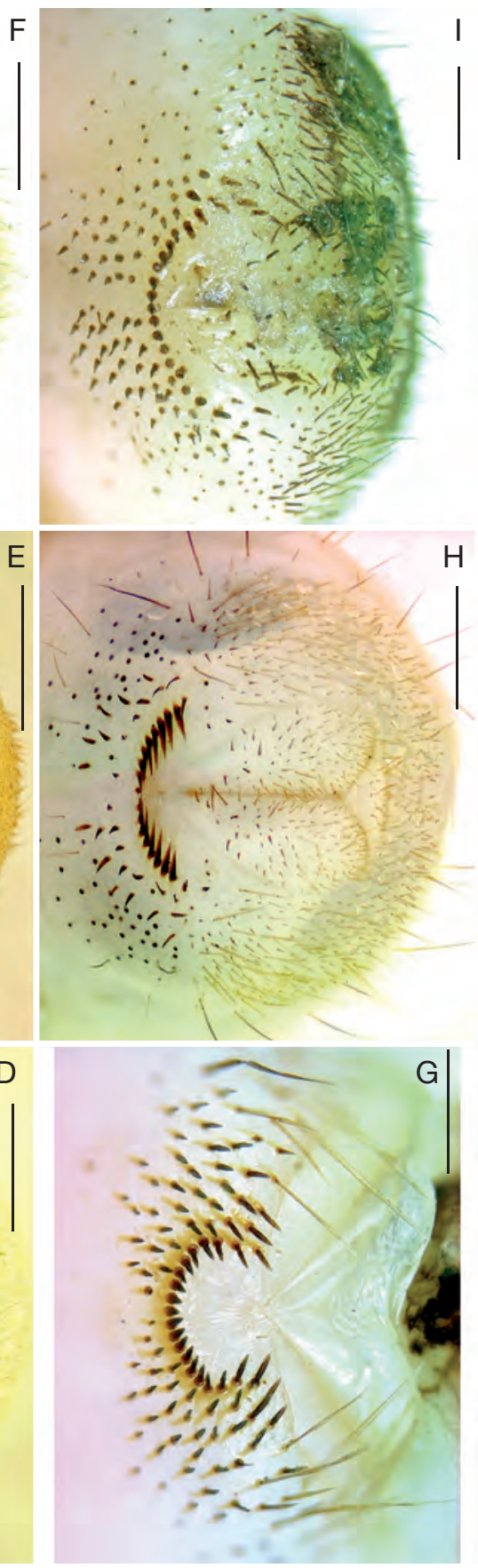

FIG. 5. - Larves de Scarabaeoidea de Madagascar, raster: A, Enaria melanictera (Klug, 1833); B, sp. M2; C, Apicencya waterloti (Dewailly, 1950); D, Empecta scutata (Fairmaire, 1901); E, Hoplochelus betanimena (Künckel, 1887); F, sp. M3; G, Triodontus nitidulus (Guérin, 1844); H, sp. S1; I, sp. S2. Échelles: A, B, F-I, 0,5 mm; C, D, 1 mm; E, 2 mm. 

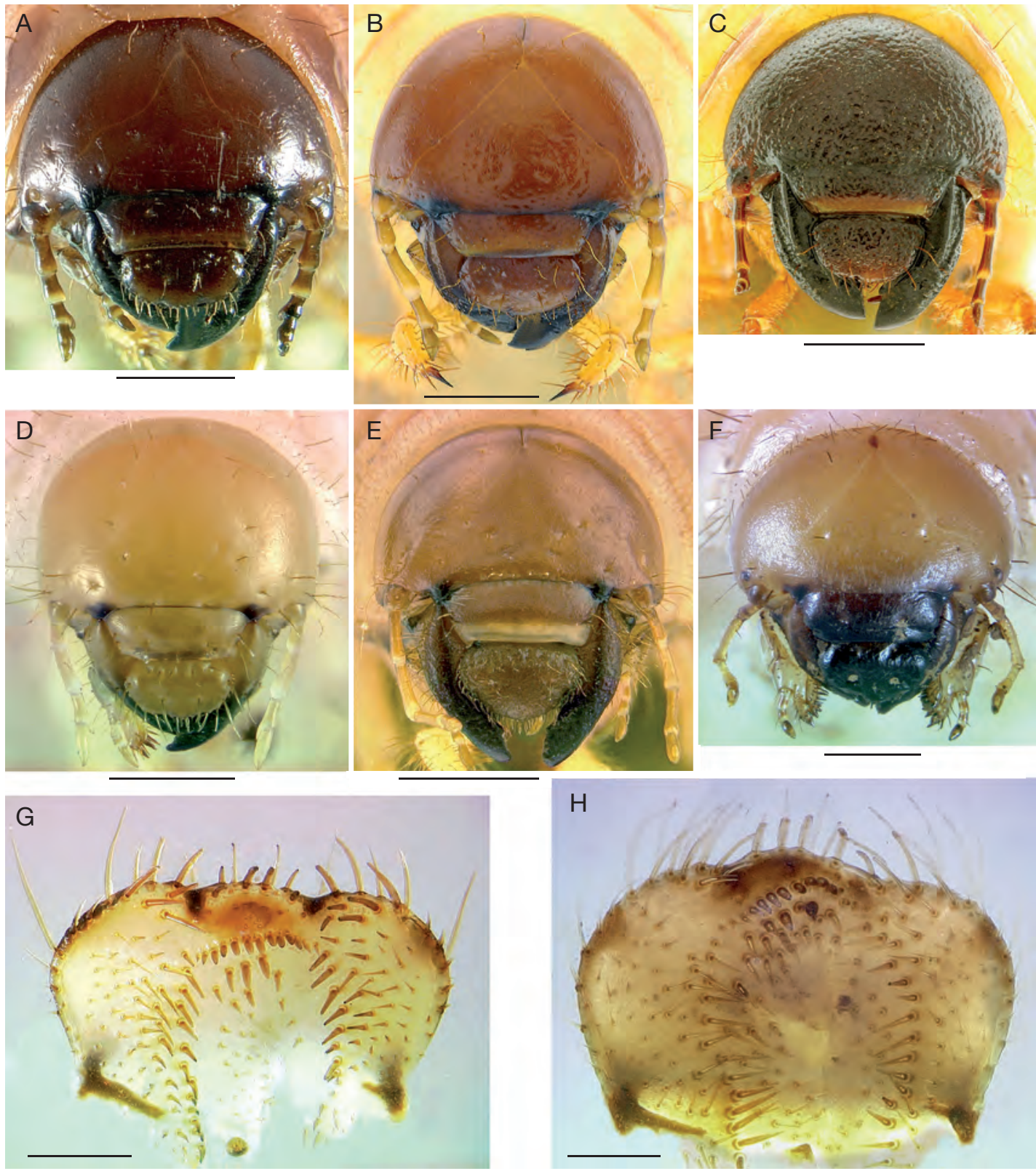

FIG. 6. - Larves de Scarabaeoidea de Madagascar: A-F, tête; A, Anochilia bifida (Olivier, 1789); B, Hexodon unicolor (Olivier, 1789); C, sp. D2; D, Paramorphochelus cornutus (Nonfried, 1892); E, Empecta scutata (Fairmaire, 1901); F, sp. S2; G, H, épipharynx; G, Bricoptis variolosa (Gory \& Percheron, 1833); H, Celidota parvula (Janson, 1881). Échelles: A-C, 2 mm; D-F, 1 mm; G, H, 0,2 mm. 

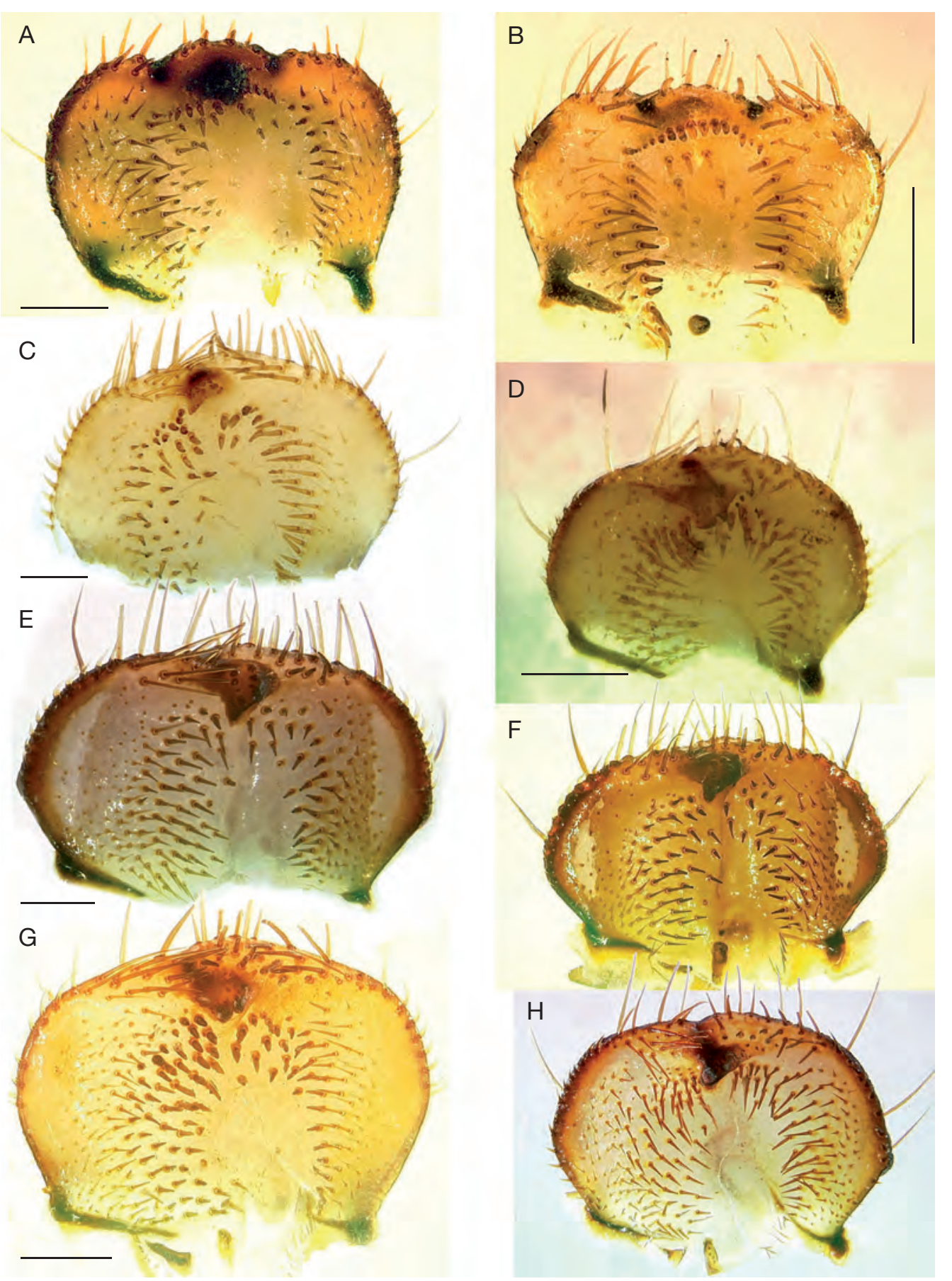

FIG. 7. - Larves de Scarabaeoidea de Madagascar, épipharynx: A, Anochilia bifida (Olivier, 1789); B, Euryomia argentea (Olivier, 1789); C, Heteronychus arator rugifrons (Fairmaire, 1871); D, Heteronychus bituberculatus (Kolbe, 1900); E, F, sp. D1; G, Hexodon unicolor (Olivier, 1789); H, sp. D2. Échelles: A, B, D, E, G, 0,5 mm; C, 0,2 mm. 


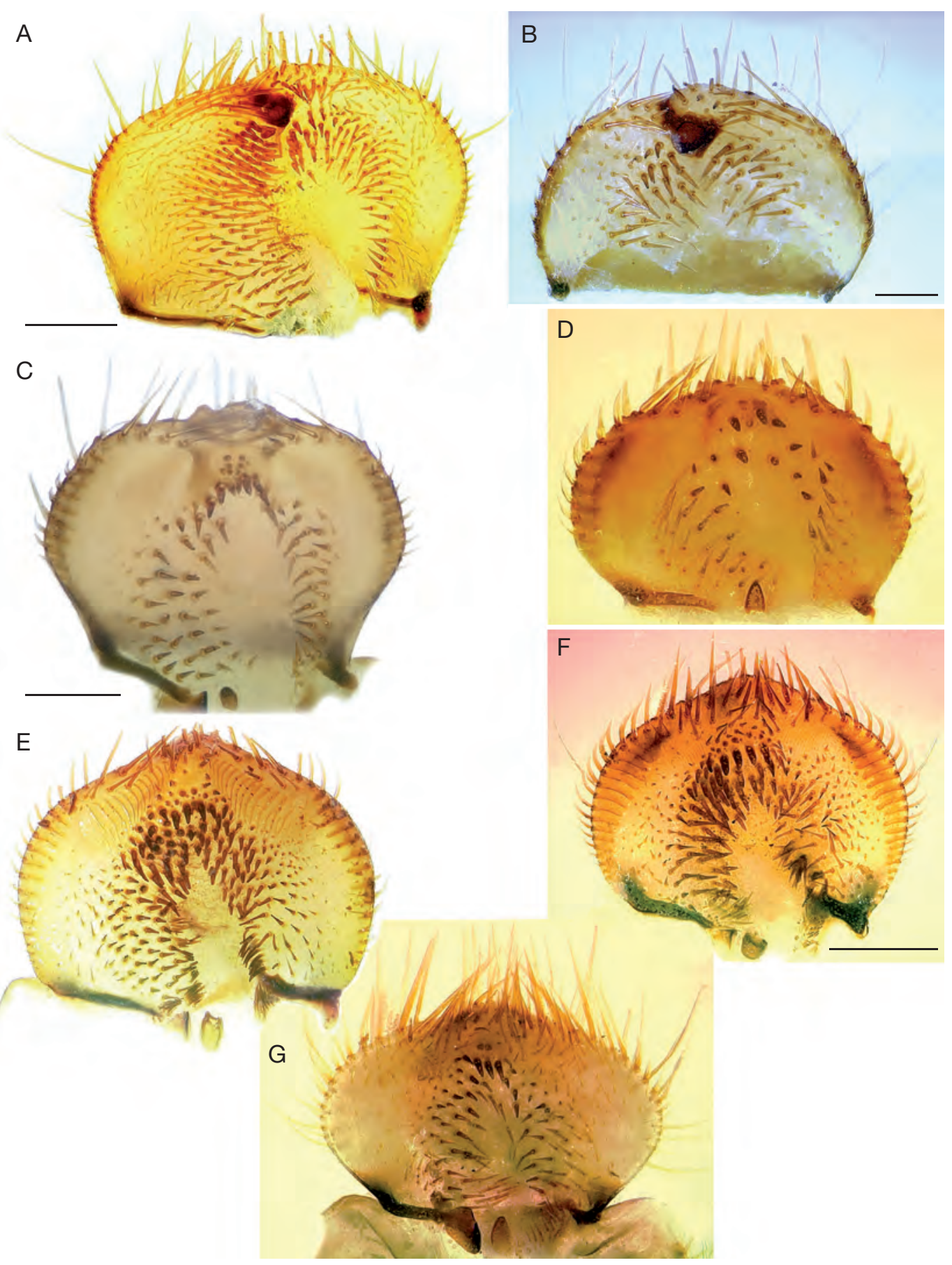

FIG. 8. - Larves de Scarabaeoidea de Madagascar, épipharynx: A, Heteroconus paradoxus (Endrödi, 1968); B, Paranodon coquerelii (Fairmaire, 1871); C, Paramorphochelus cornutus (Nonfried, 1892); D, sp. M1; E, Encya sikorai (Brenske, 1891); F, Enaria melanictera (Klug, 1833); G, sp. M2. Échelles: A, F, 0,5 mm; B, C, 0,2 mm. 


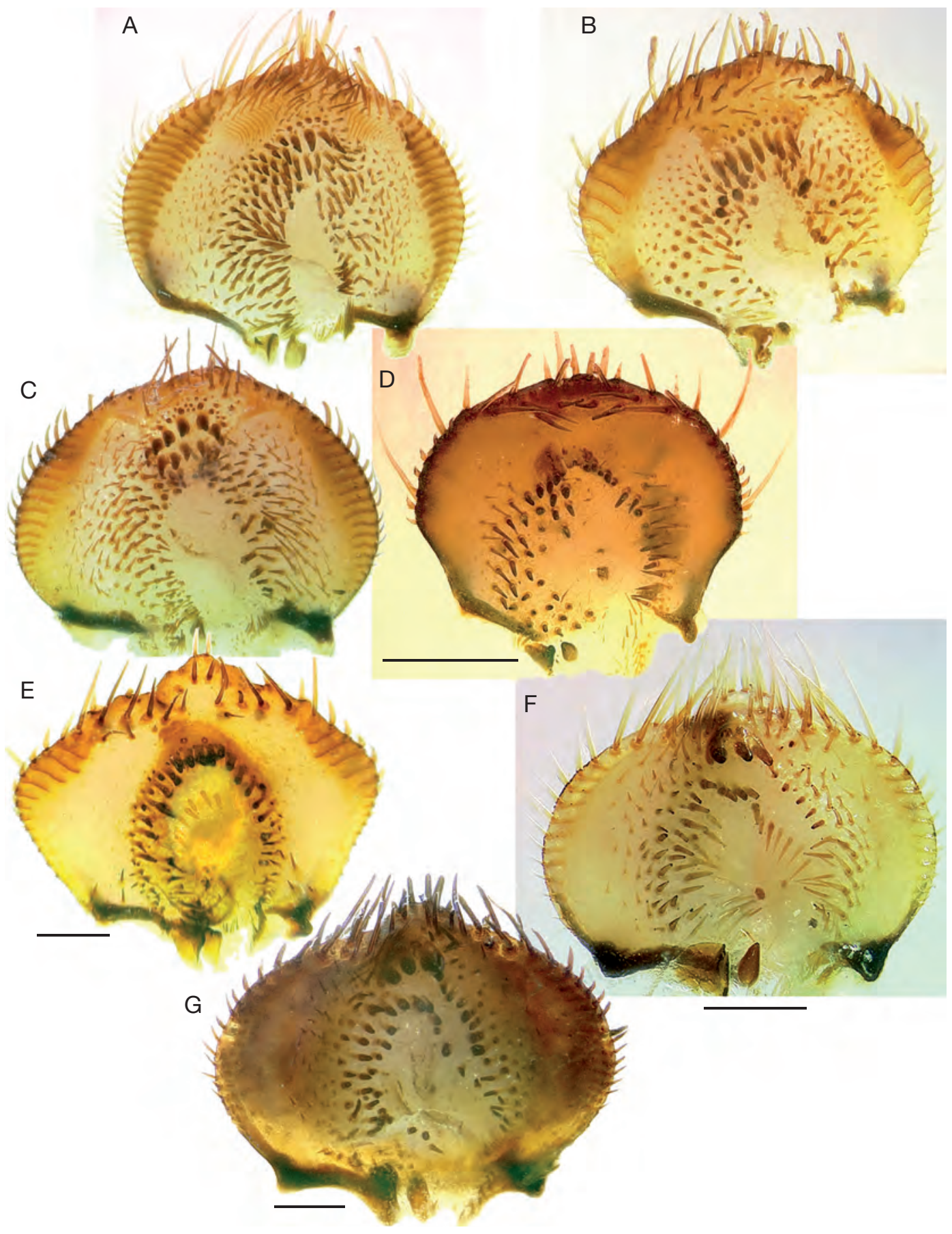

FIG. 9. - Larves de Scarabaeoidea de Madagascar, épipharynx: A, Apicencya waterloti (Dewailly, 1950); B, Empecta scutata (Fairmaire, 1901); C, Hoplochelus betanimena (Künckel, 1887); D, sp. M3; E, Triodontus nitidulus (Guérin, 1844); F, sp. S1; G, sp. S2. Échelles: $\mathrm{D}, 0,5 \mathrm{~mm}$; E-G, 0,2 mm. 

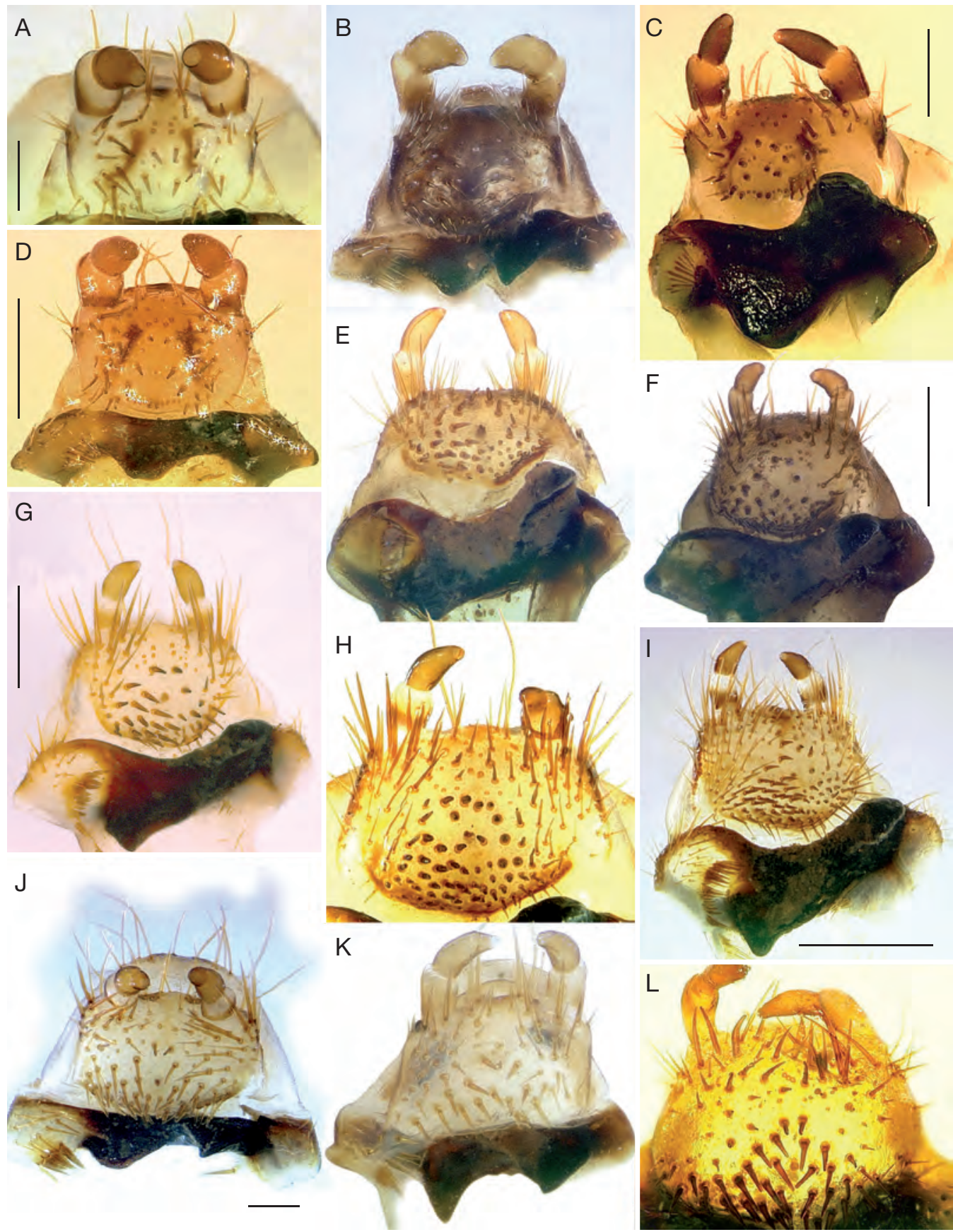

FIG. 10. - Larves de Scarabaeoidea de Madagascar, hypopharynx: A, Bricoptis variolosa (Gory \& Percheron, 1833); B, Celidota parvula (Janson, 1881); C, Anochilia bifida (Olivier, 1789); D, Euryomia argentea (Olivier, 1789); E, Heteronychus arator rugifrons (Fairmaire, 1871); F, Heteronychus bituberculatus (Kolbe, 1900); G, Heteronychus plebeius (Klug, 1833); H, Hexodon unicolor (Olivier, 1789); I, Heteroconus paradoxus (Endrödi, 1968); J, Paranodon coquerelii (Fairmaire, 1871); K, Paramorphochelus cornutus (Nonfried, 1892); L, Encya sikorai (Brenske, 1891). Échelles: A, J, 0,2 mm; C, D, F, G, I, 0,5 mm. 

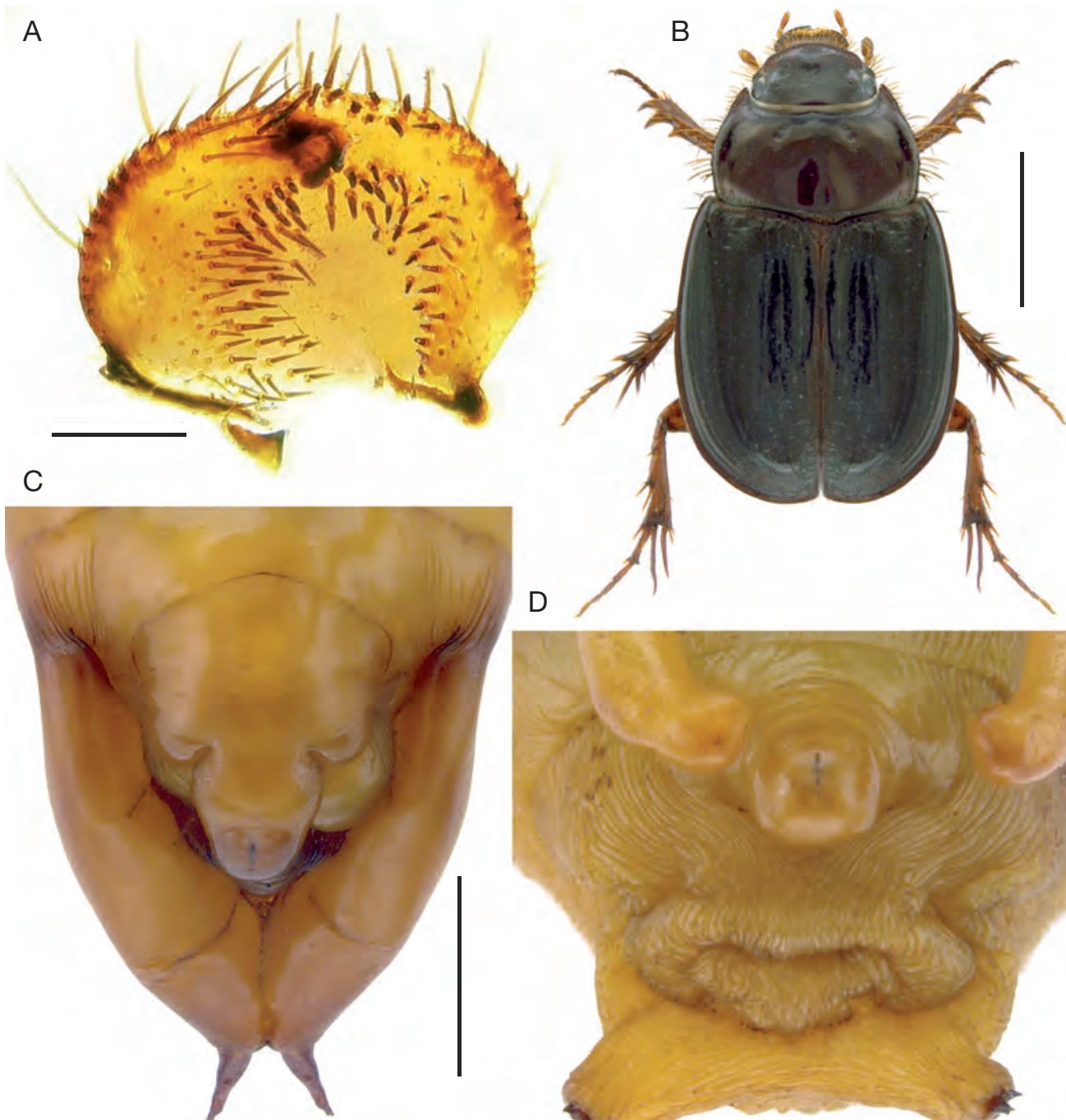

$\mathrm{F}$

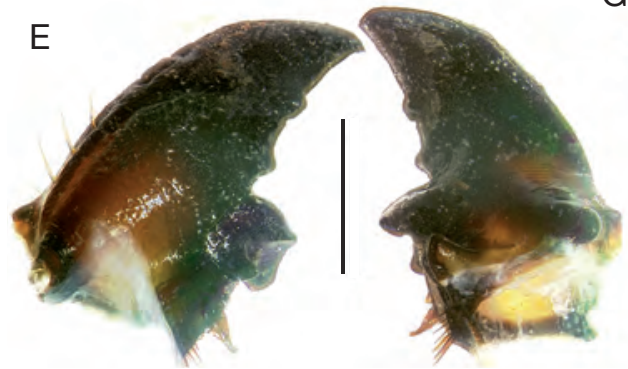

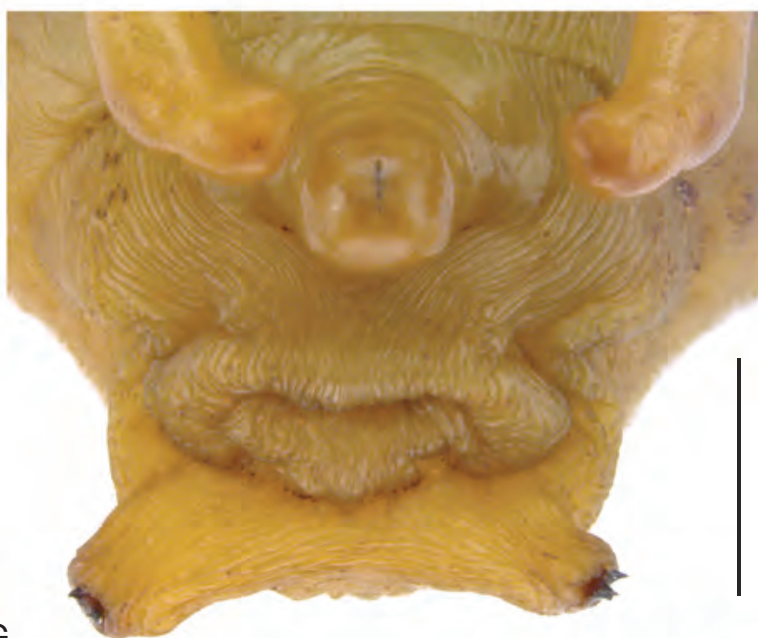

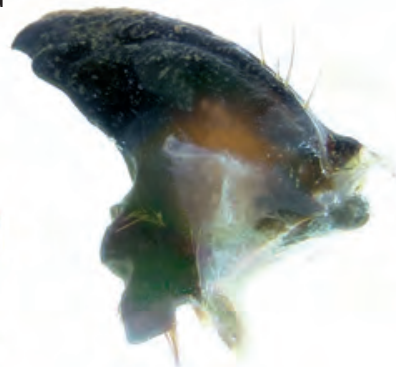

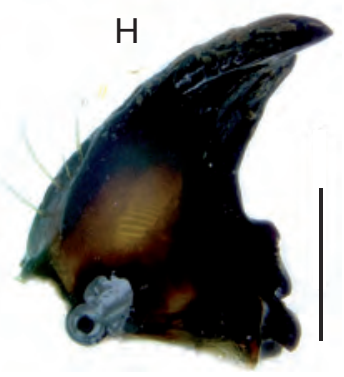

FIG. 11. - Scarabaeoidea de Madagascar: A, Heteronychus plebeius (Klug, 1833), épipharynx; B, Triodontus nitidulus (Guérin, 1844), imago, habitus; C, D, Encya sikorai (Brenske, 1891), nymphe, apex de l'abdomen, face ventrale; C, mâle; D, femelle; E-H, Heteronychus plebeius (Klug, 1833), mandibule; E, gauche, face dorsale; F, gauche, face ventrale; G, droite, face dorsale; H, droite, face ventrale. Échelles: A, E-H, 0,5 mm; B-D, 2 mm. 

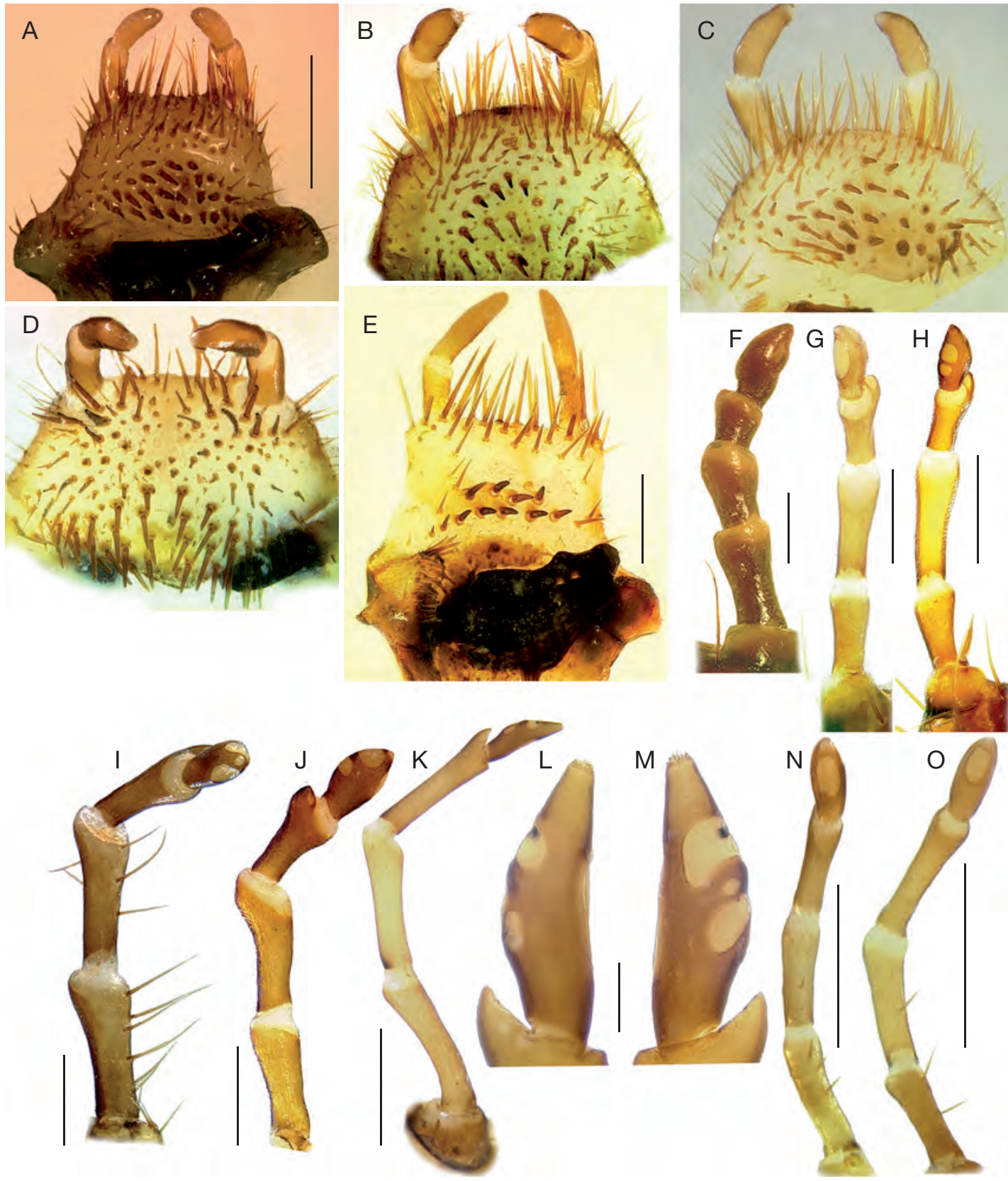

FIG. 12. - Larves de Scarabaeoidea de Madagascar: A-E, hypopharynx; A, Enaria melanictera (Klug, 1833); B, Apicencya waterloti (Dewailly, 1950); C, Empecta scutata (Fairmaire, 1901); D, Hoplochelus betanimena (Künckel, 1887); E, Triodontus nitidulus (Guérin, 1844); F-O, antenne; F, Anochilia bifida (Olivier, 1789); G, Heteronychus plebeius (Klug, 1833); H, Hexodon unicolor (Olivier, 1789); I, Heteroconus paradoxus (Endrödi, 1968); J, Paranodon coquerelii (Fairmaire, 1871); K-M, Encya sikorai (Brenske, 1891); L, M, plages sensorielles de l'article apical; N, Apicencya waterloti (Dewailly, 1950); O, Empecta scutata (Fairmaire, 1901). Échelles: A, F, G, I, J, $\mathrm{O}, 0,5 \mathrm{~mm} ; \mathrm{E}, \mathrm{L}, \mathrm{M}, 0,2 \mathrm{~mm} ; \mathrm{H}, \mathrm{K}, \mathrm{N}, 1 \mathrm{~mm}$ 


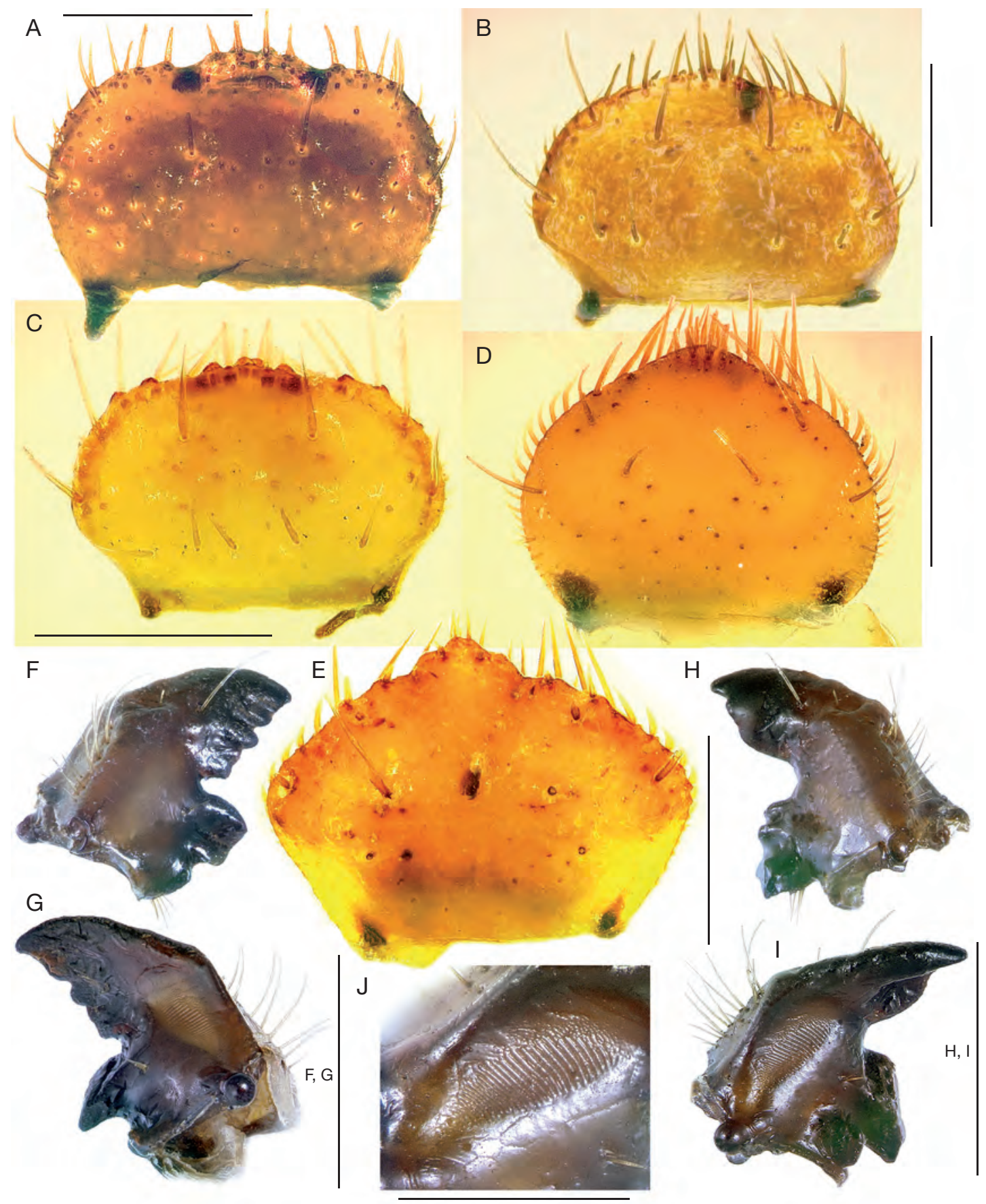

FIG. 13. - Larves de Scarabaeoidea de Madagascar: A-E, labre; A, Anochilia bifida (Olivier, 1789); B, Heteronychus plebeius (Klug, 1833); C, Paramorphochelus cornutus (Nonfried, 1892); D, Enaria melanictera (Klug, 1833); E, Triodontus nitidulus (Guérin, 1844); F-J, Celidota parvula (Janson, 1881), mandibule; F, gauche, face dorsale; G, gauche, face ventrale; H, droite, face dorsale; I, droite, face ventrale; J, aire stridulatoire mandibulaire. Échelles: A, B, D, F-I, $1 \mathrm{~mm}$; C, E, J, 0,5 mm. 

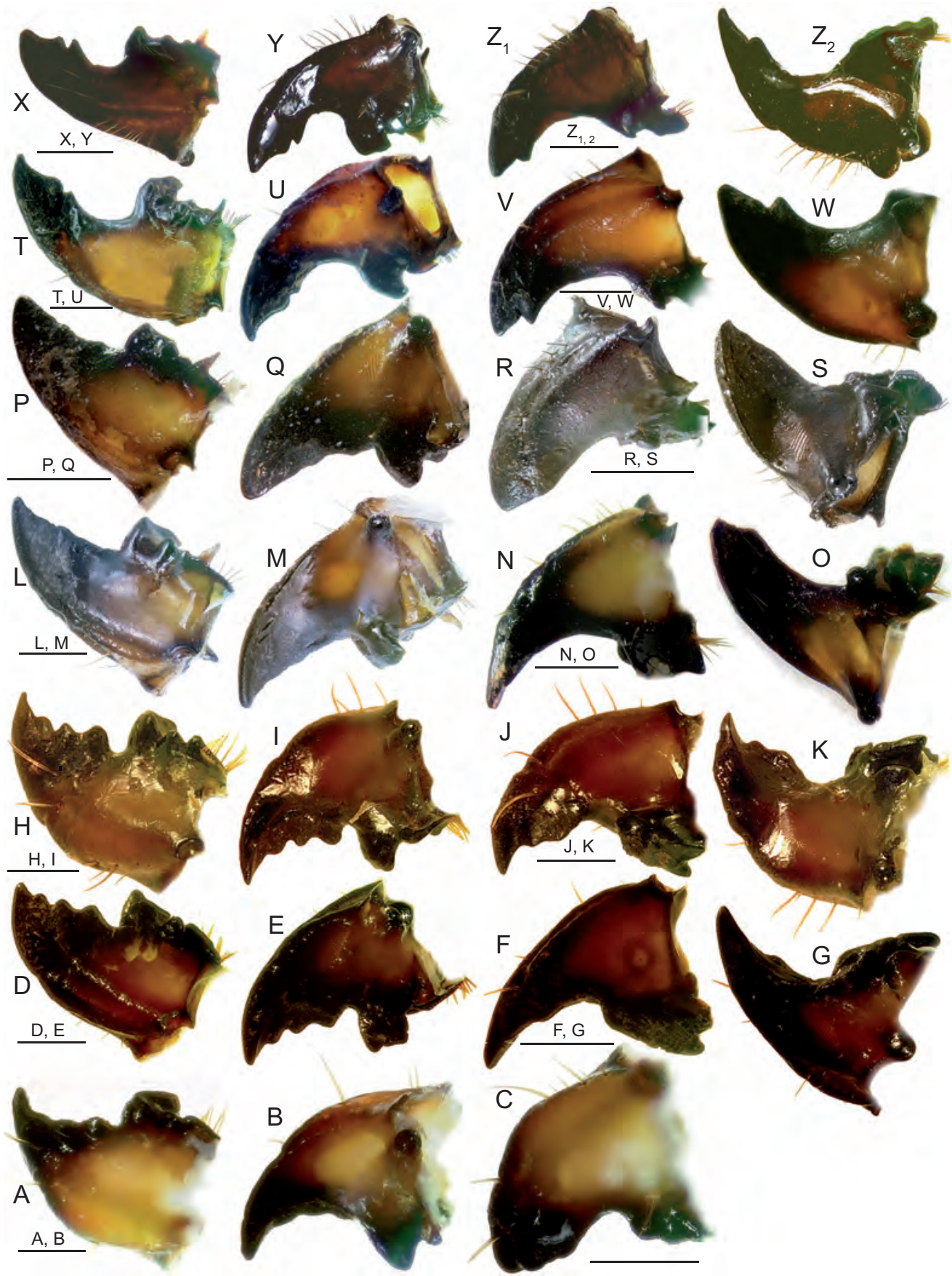

FIG. 14. - Larves de Scarabaeoidea de Madagascar, mandibule: A, D, H, L, P, T, X, gauche, face dorsale; B, E, I, M, Q, U, Y, gauche, face ventrale; C, F, J, N, R, V, Z 1 , droite, face dorsale; G, K, O, S, W, Z 2 , droite, face ventrale; A-C, Bricoptis variolosa (Gory \& Percheron, 1833); D-G, Anochilia bifida (Olivier, 1789); H-K, Euryomia argentea (Olivier, 1789); L-O, Heteronychus arator rugifrons (Fairmaire, 1871); P-S, Heteronychus bituberculatus (Kolbe, 1900); T-W, Hexodon unicolor (Olivier, 1789); X-Z $\mathbf{Z}_{2}$, Heteroconus paradoxus (Endrödi, 1968). Échelles: A-C, H-O, 0,5 mm; D-G, P-Z, 1 mm. 


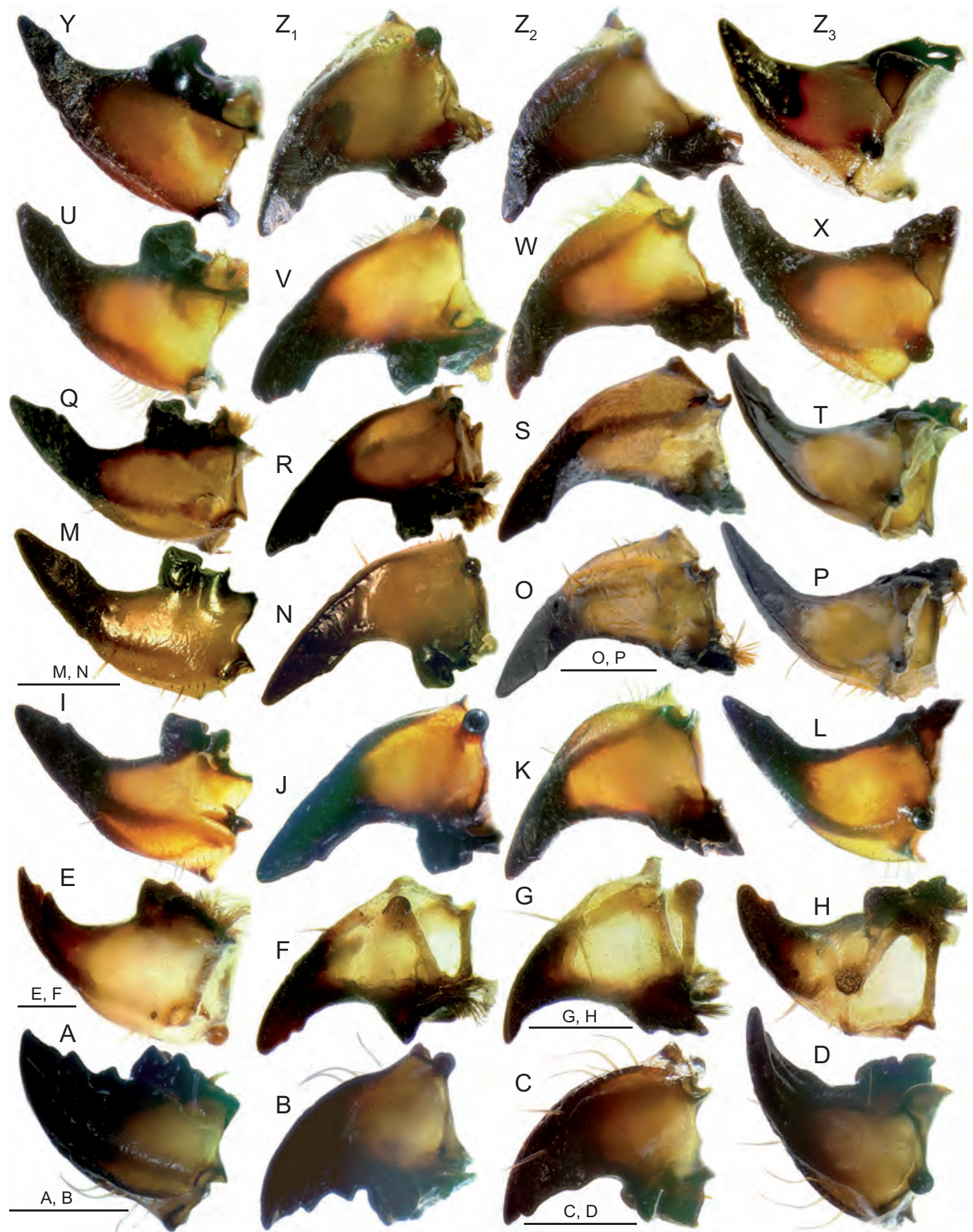

FIG. 15. - Larves de Scarabaeoidea de Madagascar, mandibule: A, E, I, M, Q, U, Y, gauche, face dorsale; B, F, J, N, R, V, Z 1 , gauche, face ventrale; C, G, K, O, S, W, $\mathbf{Z}_{\mathbf{2}}$, droite, face dorsale; D, H, L, P, T, X, Z $\mathbf{3}$, droite, face ventrale; A-D, Paranodon coquerelii (Fairmaire, 1871); E-H, Paramorphochelus cornutus (Nonfried, 1892); I-L, Encya sikorai (Brenske, 1891); M-P, Enaria melanictera (Klug, 1833); Q-T, Apicencya waterloti (Dewailly, 1950); U-X, Empecta scutata (Fairmaire, 1901); Y-Z, , Hoplochelus betanimena (Künckel, 1887). Échelles: A-D, M-P, $1 \mathrm{~mm}$; E-H, 0,5 mm. 


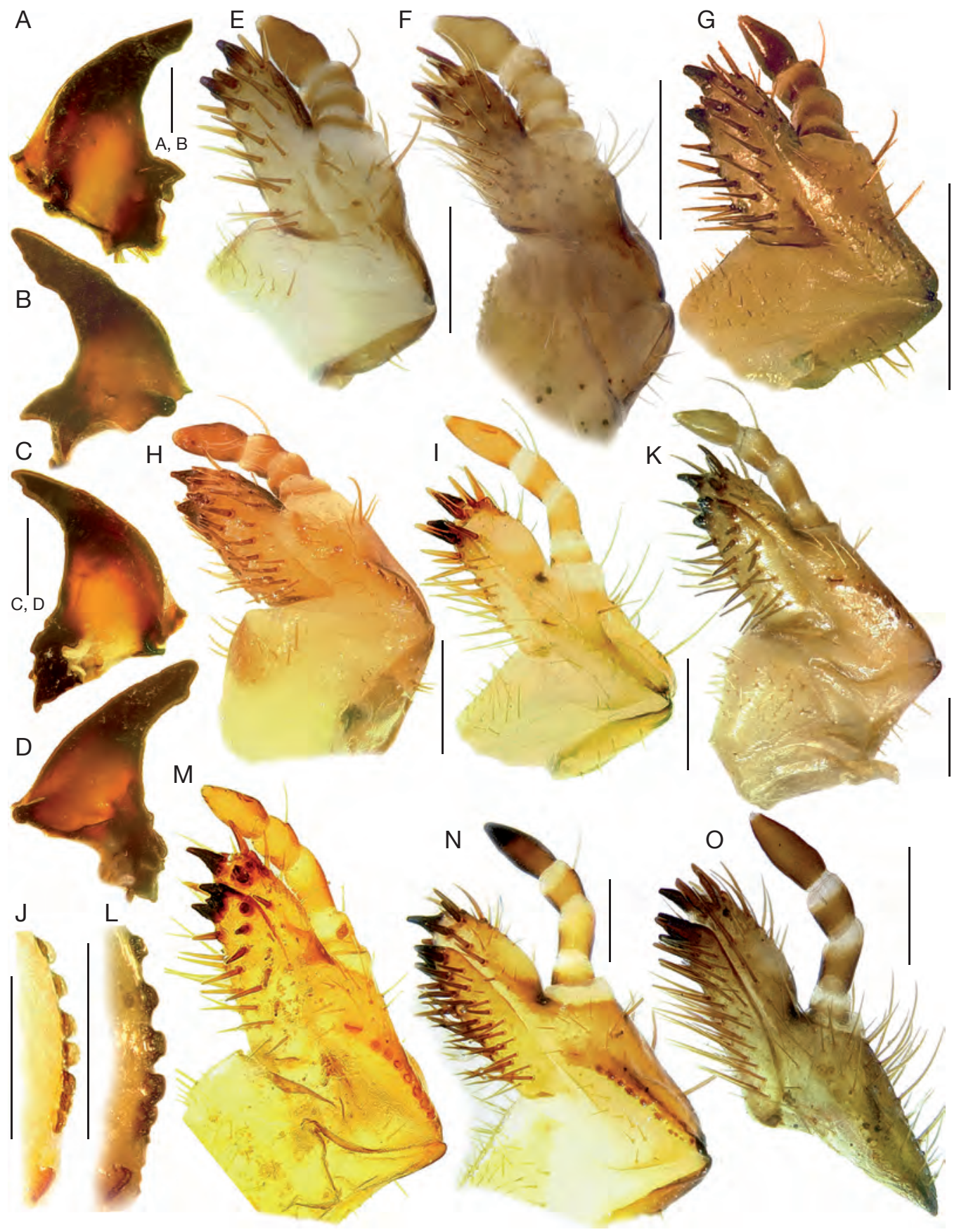

FIG. 16. - Larves de Scarabaeoidea de Madagascar: A-D, mandibule, Triodontus nitidulus (Guérin, 1844); A, gauche, face dorsale; B, gauche, face ventrale; C, droite, face dorsale; D, droite, face ventrale; E-O, maxille, face dorsale; E, Bricoptis variolosa (Gory \& Percheron, 1833); F, Celidota parvula (Janson, 1881); G, Anochilia bifida (Olivier, 1789); H, Euryomia argentea (Olivier, 1789); I, J, Heteronychus arator rugifrons (Fairmaire, 1871); J, dents stridulatoires; K, L, Heteronychus bituberculatus (Kolbe, 1900); L, dents stridulatoires; M, Heteronychus plebeius (Klug, 1833); N, Hexodon unicolor (Olivier, 1789); O, Heteroconus paradoxus (Endrödi, 1968). Échelles: A-D, N, O, 0,5 mm; E, F, H, I, K, 0,4 mm; G, $1 \mathrm{~mm}$; J, 0,01 mm; L, 0,02 mm. 


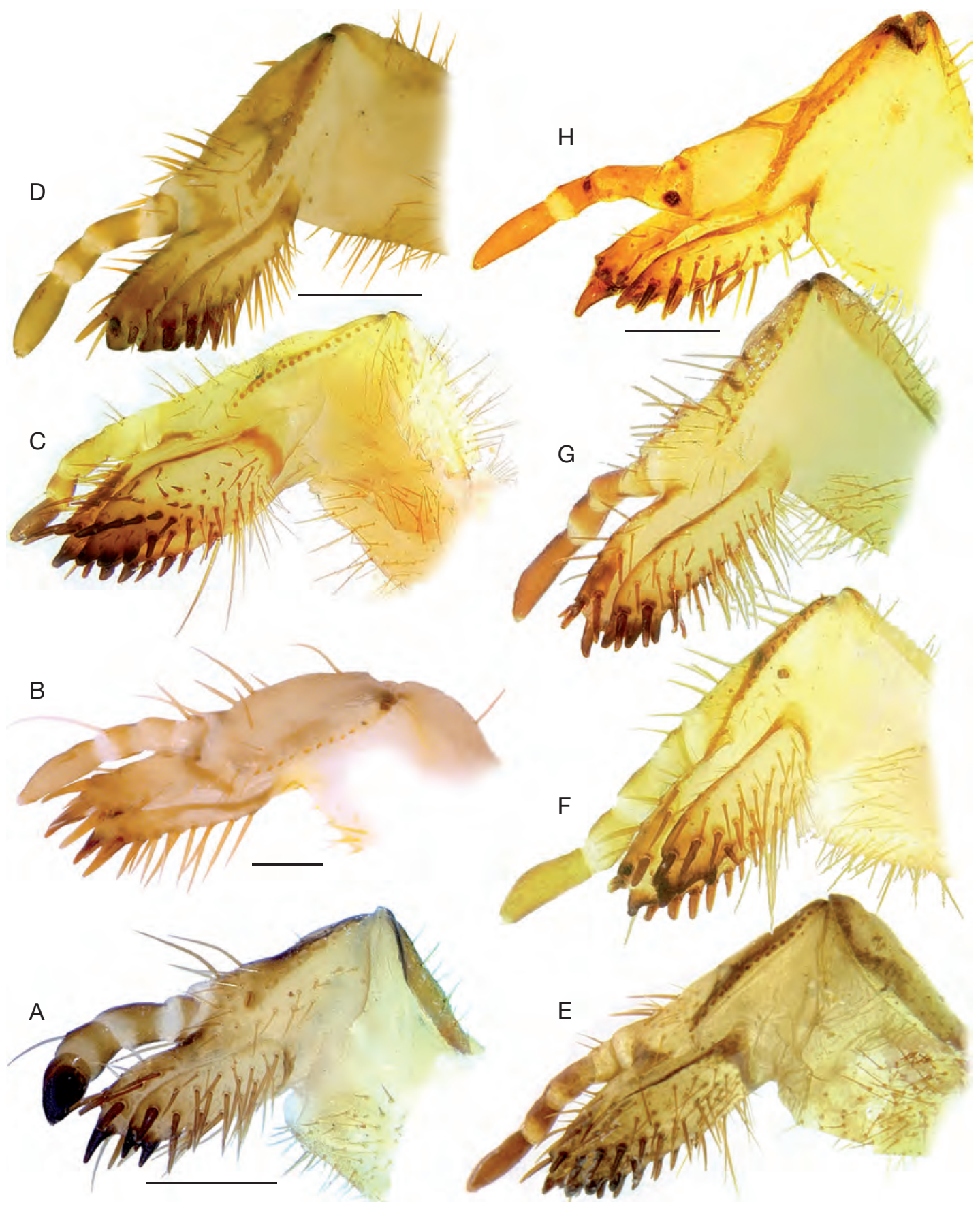

FIG. 17. - Larves de Scarabaeoidea de Madagascar, maxille, face dorsale: A, Paranodon coquerelii (Fairmaire, 1871); B, Paramorphochelus cornutus (Nonfried, 1892); C, Encya sikorai (Brenske, 1891); D, Enaria melanictera (Klug, 1833); E, Apicencya waterloti (Dewailly, 1950); F, Empecta scutata (Fairmaire, 1901); G, Hoplochelus betanimena (Künckel, 1887); H, Triodontus nitidulus (Guérin, 1844). Échelles: A, 0,5 mm; B, 0,1 mm; D, H, 0,2 mm. 


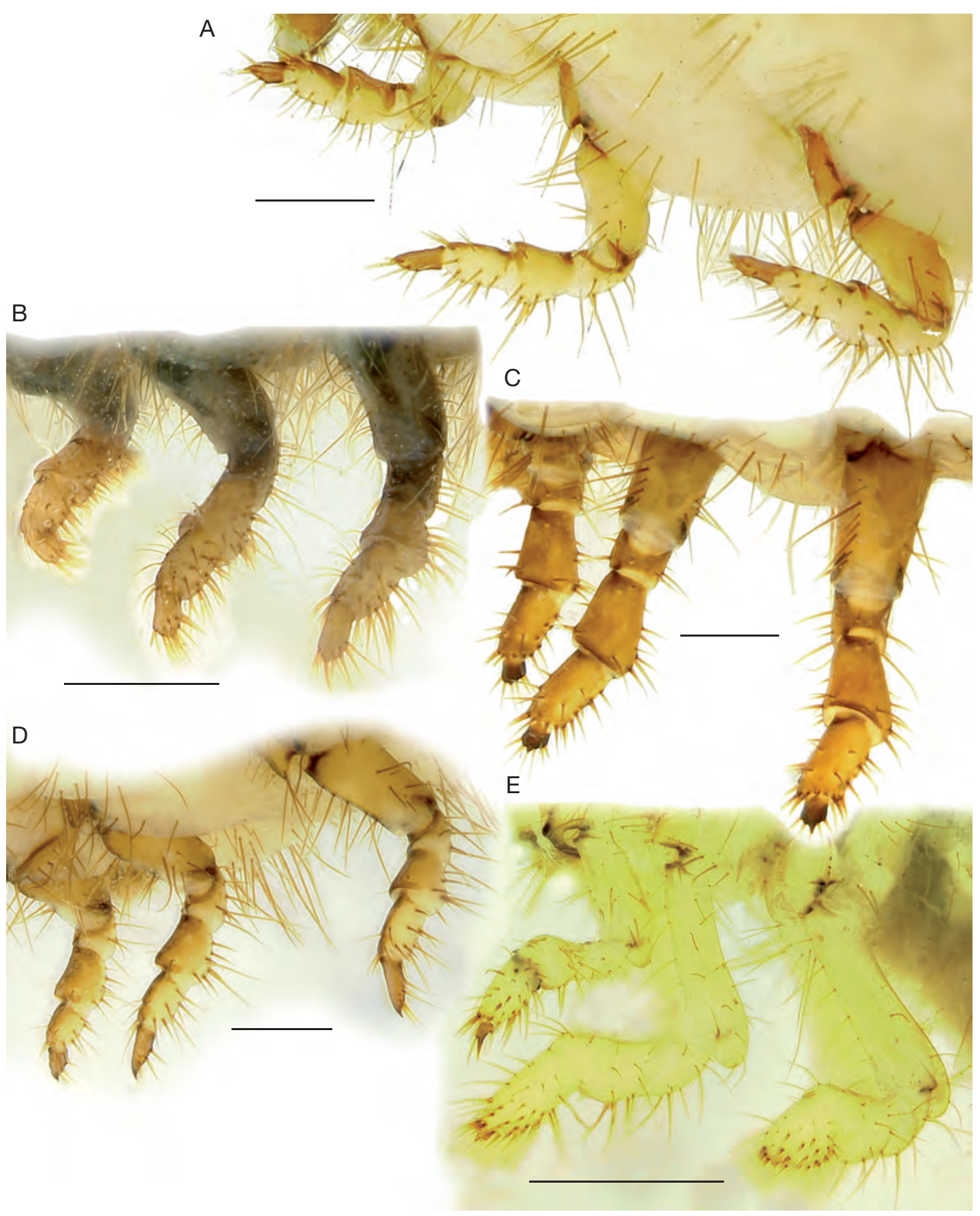

FIG. 18. - Larves de Scarabaeoidea de Madagascar, pattes: A, Bricoptis variolosa (Gory \& Percheron, 1833); B, Celidota parvula (Janson, 1881); C, Anochilia bifida (Olivier, 1789); D, Euryomia argentea (Olivier, 1789); E, Paramorphochelus cornutus (Nonfried, 1892). Échelles: $1 \mathrm{~mm}$. 


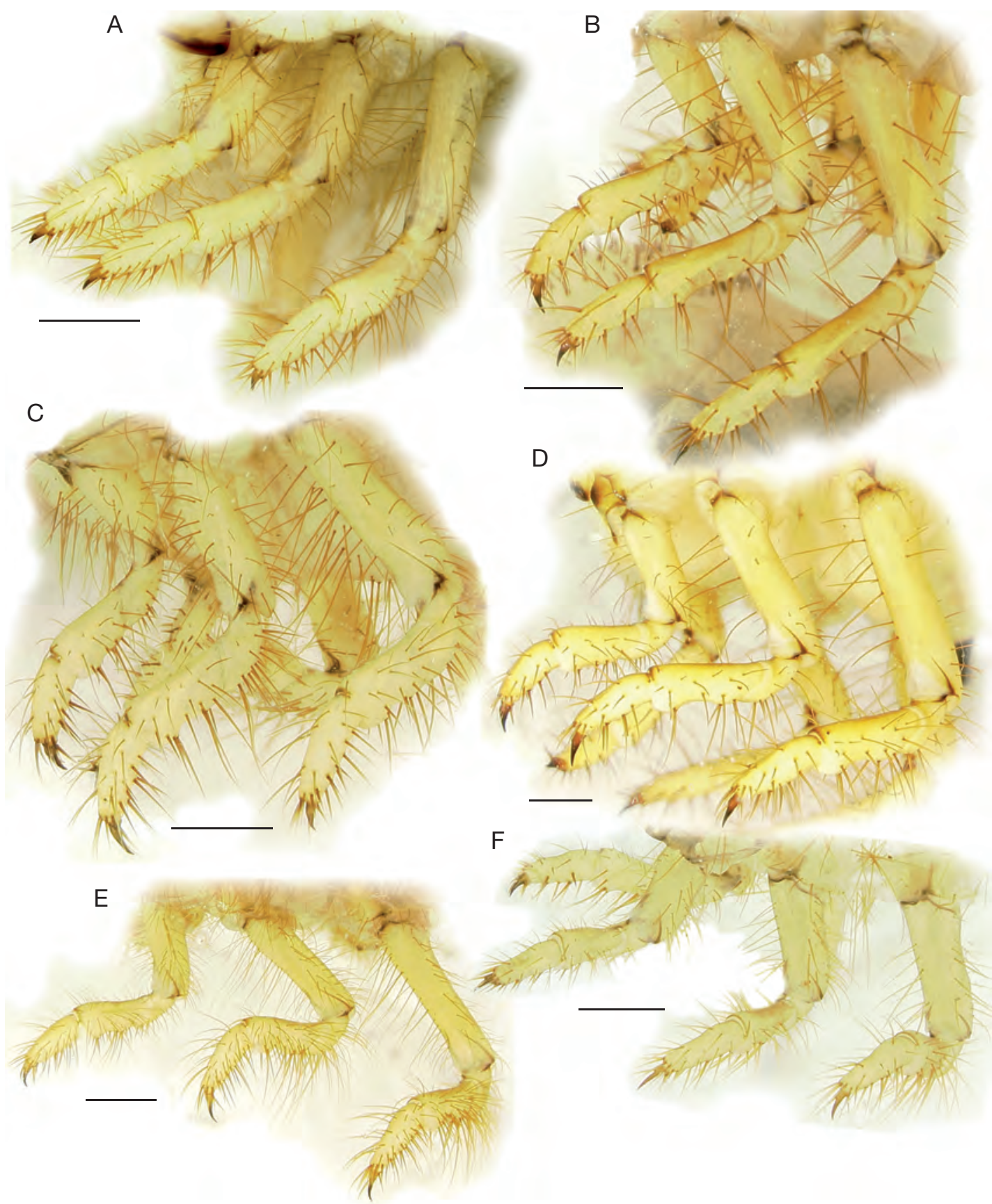

FIG. 19. - Larves de Scarabaeoidea de Madagascar, pattes: A, Heteronychus arator rugifrons (Fairmaire, 1871); B, Heteronychus bituberculatus (Kolbe, 1900); C, Heteronychus plebeius (Klug, 1833); D, Hexodon unicolor (Olivier, 1789); E, Heteroconus paradoxus (Endrödi, 1968); F, Paranodon coquerelii (Fairmaire, 1871). Échelles: $1 \mathrm{~mm}$. 

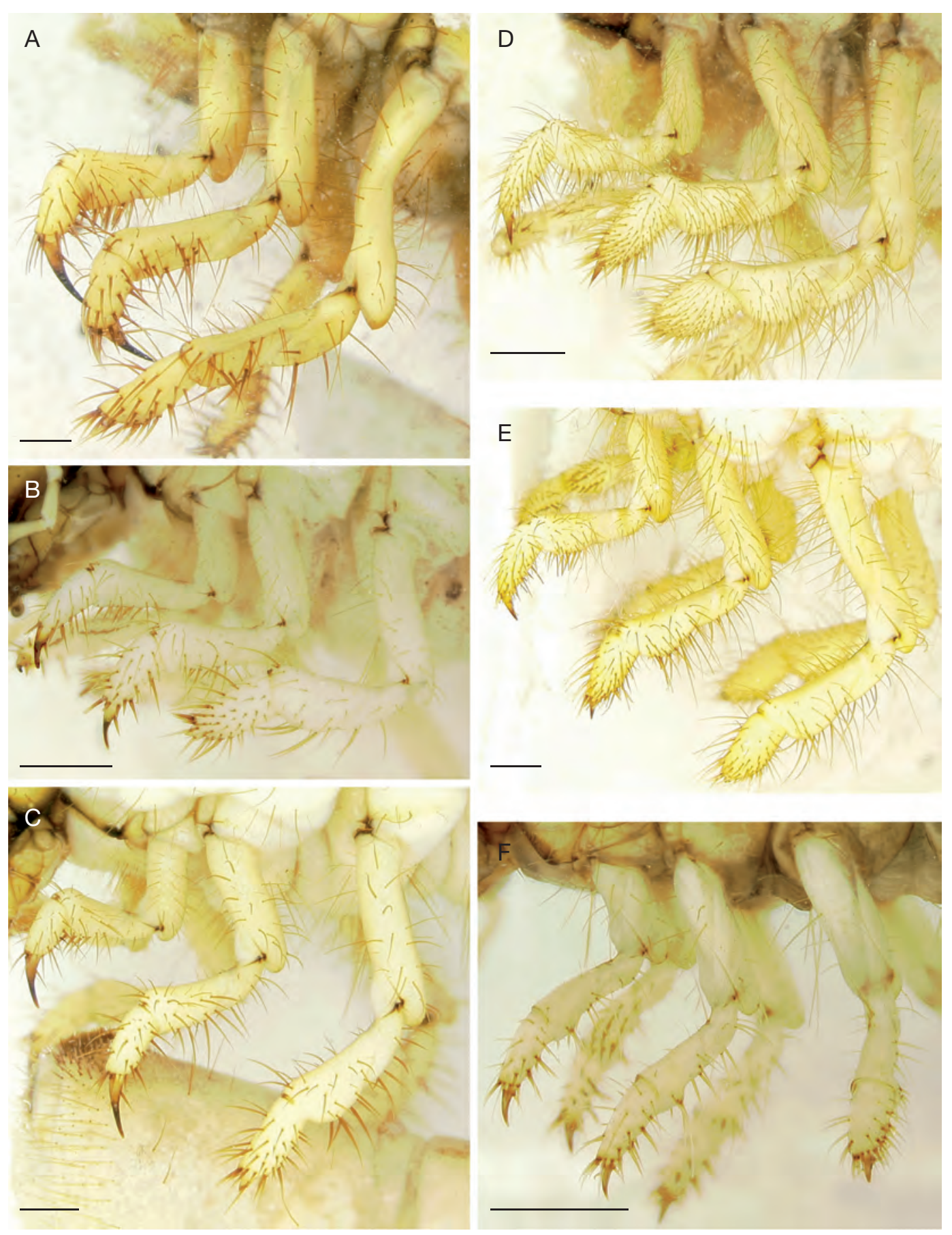

FIG. 20. - Larves de Scarabaeoidea de Madagascar, pattes: A, Encya sikorai (Brenske, 1891); B, Enaria melanictera (Klug, 1833); C, Apicencya waterloti (Dewailly, 1950); D, Empecta scutata (Fairmaire, 1901); E, Hoplochelus betanimena (Künckel, 1887); F, Triodontus nitidulus (Guérin, 1844). Échelles: $1 \mathrm{~mm}$. 

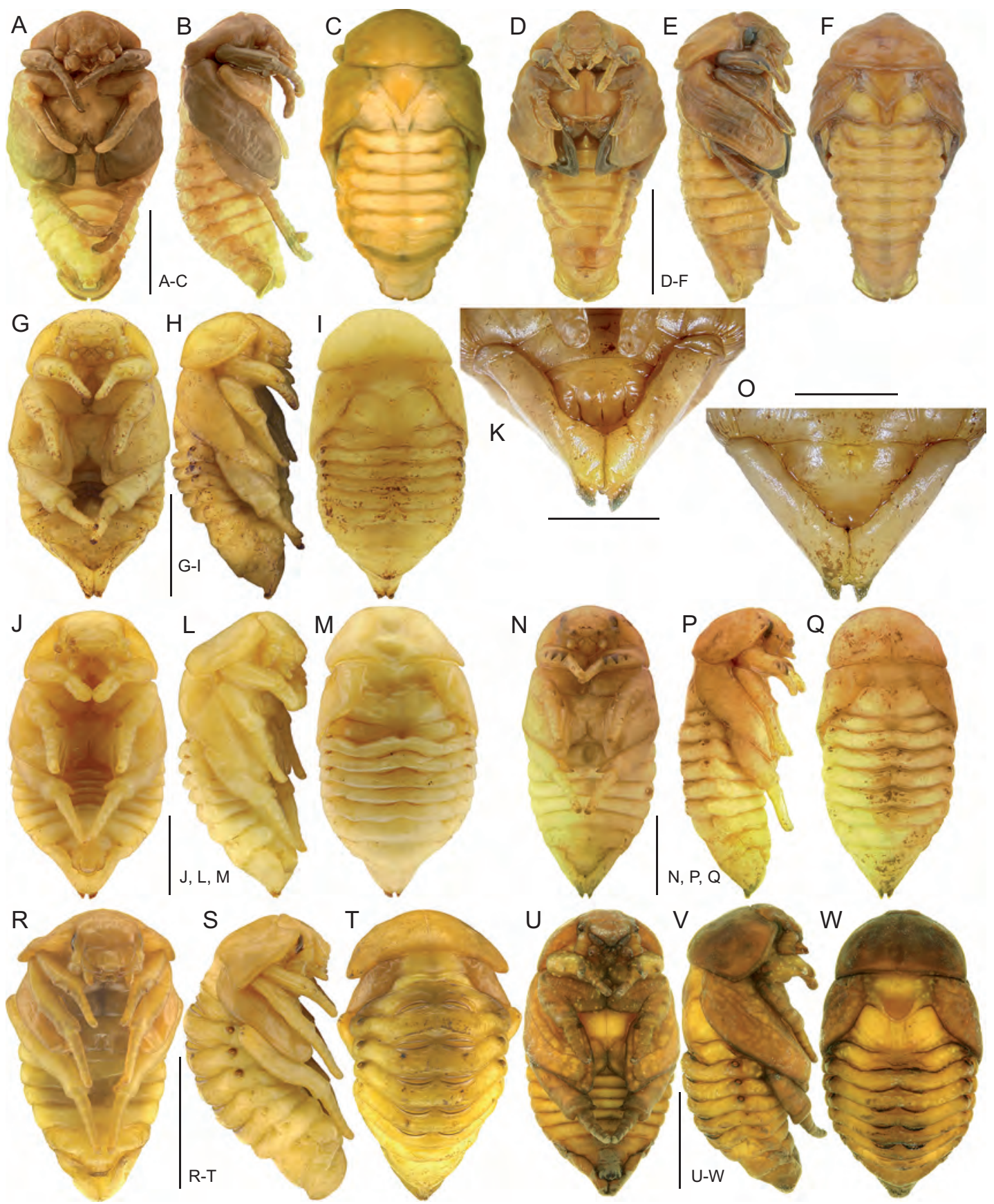

FIG. 21. - Nymphes de Scarabaeoidea de Madagascar: A-C, Bricoptis variolosa (Gory \& Percheron, 1833); D-F, Euryomia argentea (Olivier, 1789); G-I, Heteronychus arator rugifrons (Fairmaire, 1871); J-M, Heteronychus plebeius (Klug, 1833) mâle; K, apex de l'abdomen; $\mathbf{N}-\mathbf{Q}$, Heteronychus plebeius (Klug, 1833) femelle; O, apex de l'abdomen; R-T, Hexodon unicolor (Olivier, 1789); U-W, Heteroconus paradoxus (Endrödi, 1968); A, D, G, J, N, R, U, faces ventrales; B, E, H, L, P, S, V, faces latérales; C, F, I, M, Q, T, W, W, faces dorsales. Échelles: A-J, L-N, P, Q, U-W, $5 \mathrm{~mm}$; K, O, $2 \mathrm{~mm}$; R-T, $10 \mathrm{~mm}$. 

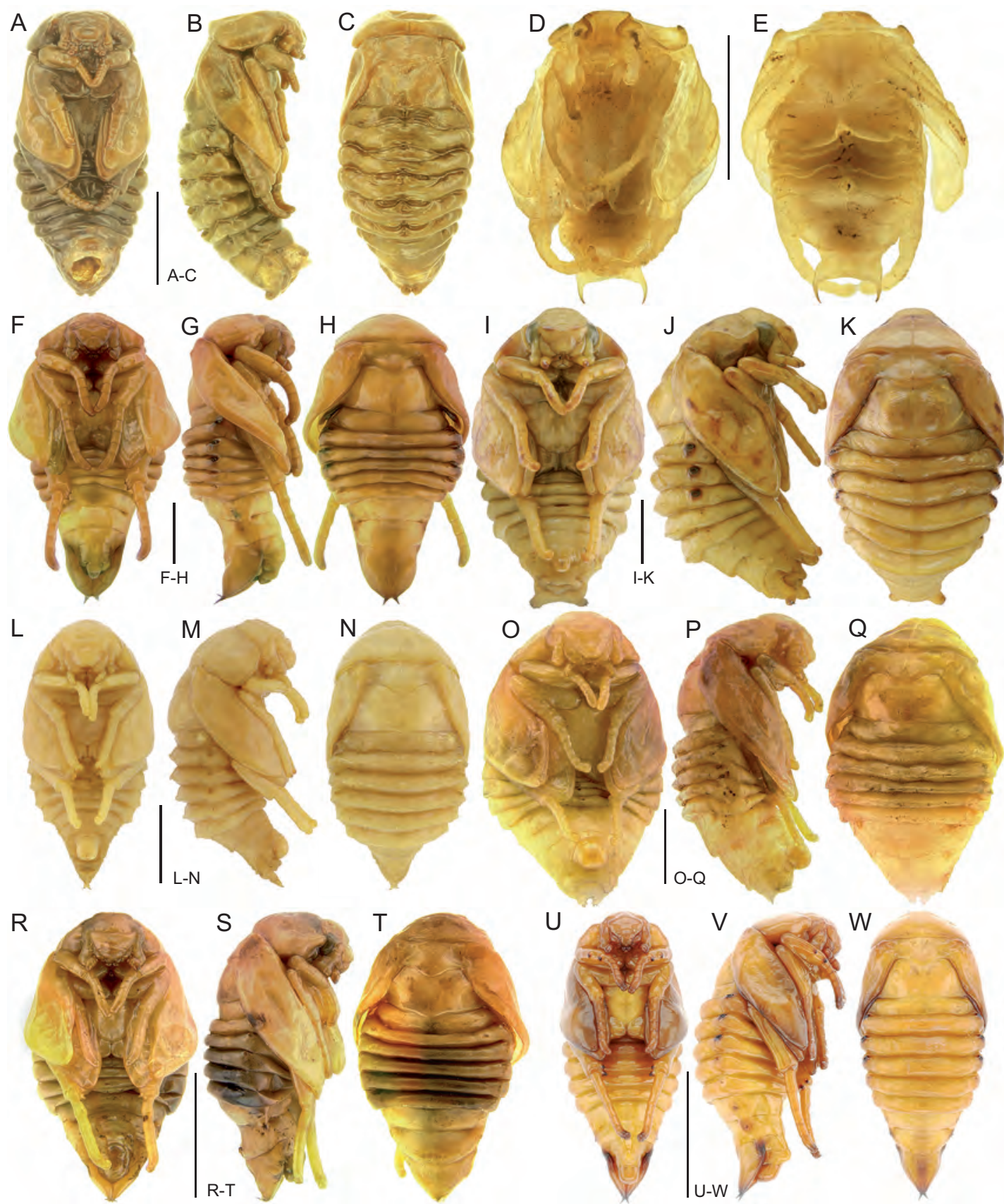

FIG. 22. - Nymphes de Scarabaeoidea de Madagascar: A-C, Paranodon coquerelii (Fairmaire, 1871); A, spécimen endommagé à I'apex abdominal; D, E, Paramorphochelus cornutus (Nonfried, 1892); F-H, Encya sikorai (Brenske, 1891) mâle; I-K, Encya sikorai (Brenske, 1891) femelle; L-N, Enaria melanictera (Klug, 1833); O-Q, Apicencya waterloti (Dewailly, 1950); R-T, Empecta scutata (Fairmaire, 1901); U-W, Hoplochelus betanimena (Künckel, 1887); A, D, F, I, L, O, R, U, faces ventrales; B, G, J, M, P, S, V, faces latérales; C, E, H, K, N, Q, T, W, faces dorsales. Échelles: A-T, 5 mm; U-W, 10 mm. 

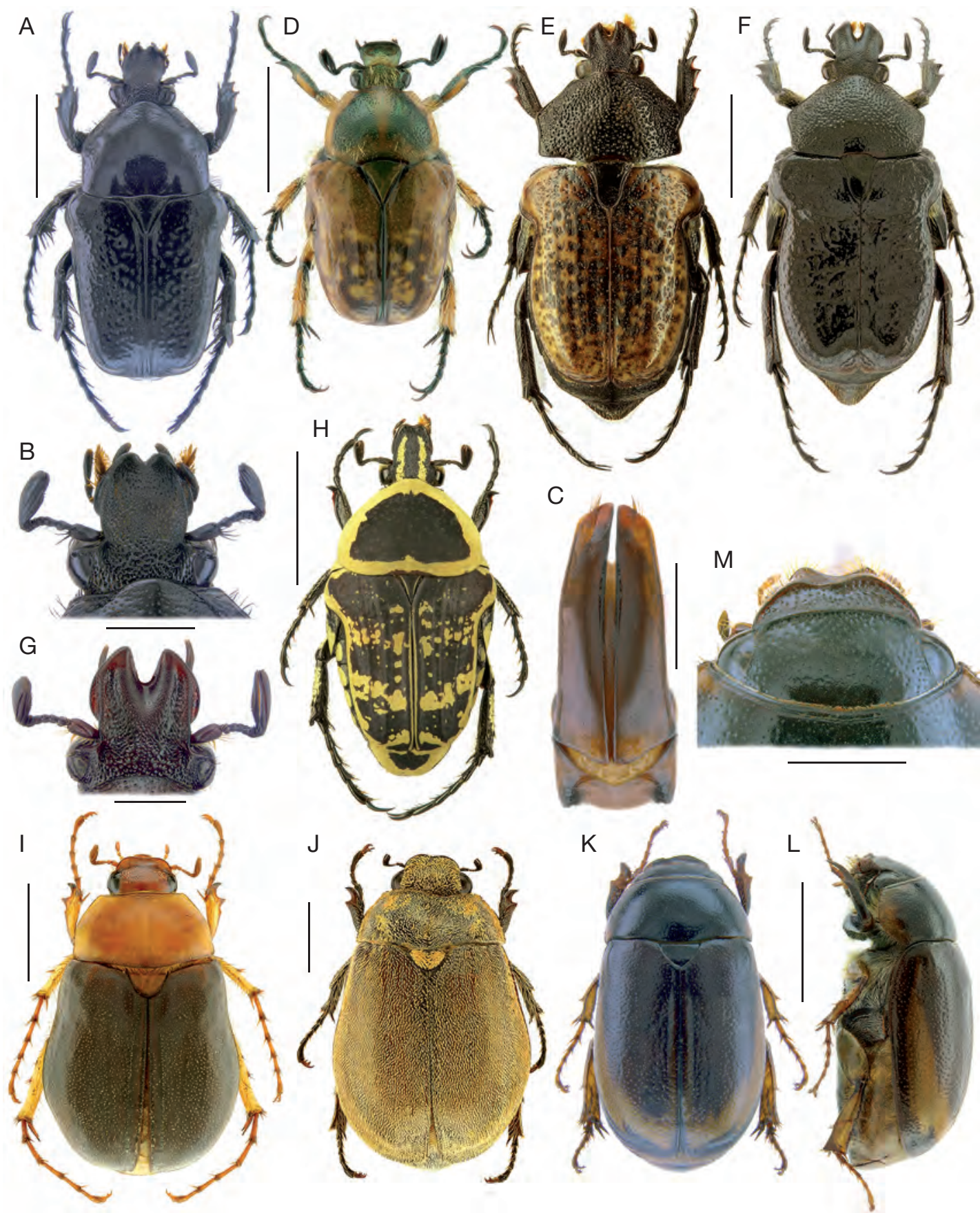

FIG. 23. - Imagos de Scarabaeoidea de Madagascar: A-C, Bricoptis variolosa (Gory \& Percheron, 1833); A, habitus; B, tête, face dorsale; C, édéage; D, Celidota parvula (Janson, 1881), habitus; E-G, Anochilia bifida (Olivier, 1789); E, F, habitus; G, tête, face dorsale; H, Euryomia argentea (Olivier, 1789), habitus; I, Hoplochelus betanimena (Künckel, 1887), habitus; J, Encya sikorai (Brenske, 1891), habitus; K-M, Enaria melanictera (Klug, 1833); K, habitus; L, face latérale; M, tête, face dorsale. Échelles: A, D, E, F-L, 5 mm; $\mathrm{B}, \mathrm{G}, \mathrm{M}, 2 \mathrm{~mm}$; C, $1 \mathrm{~mm}$. 


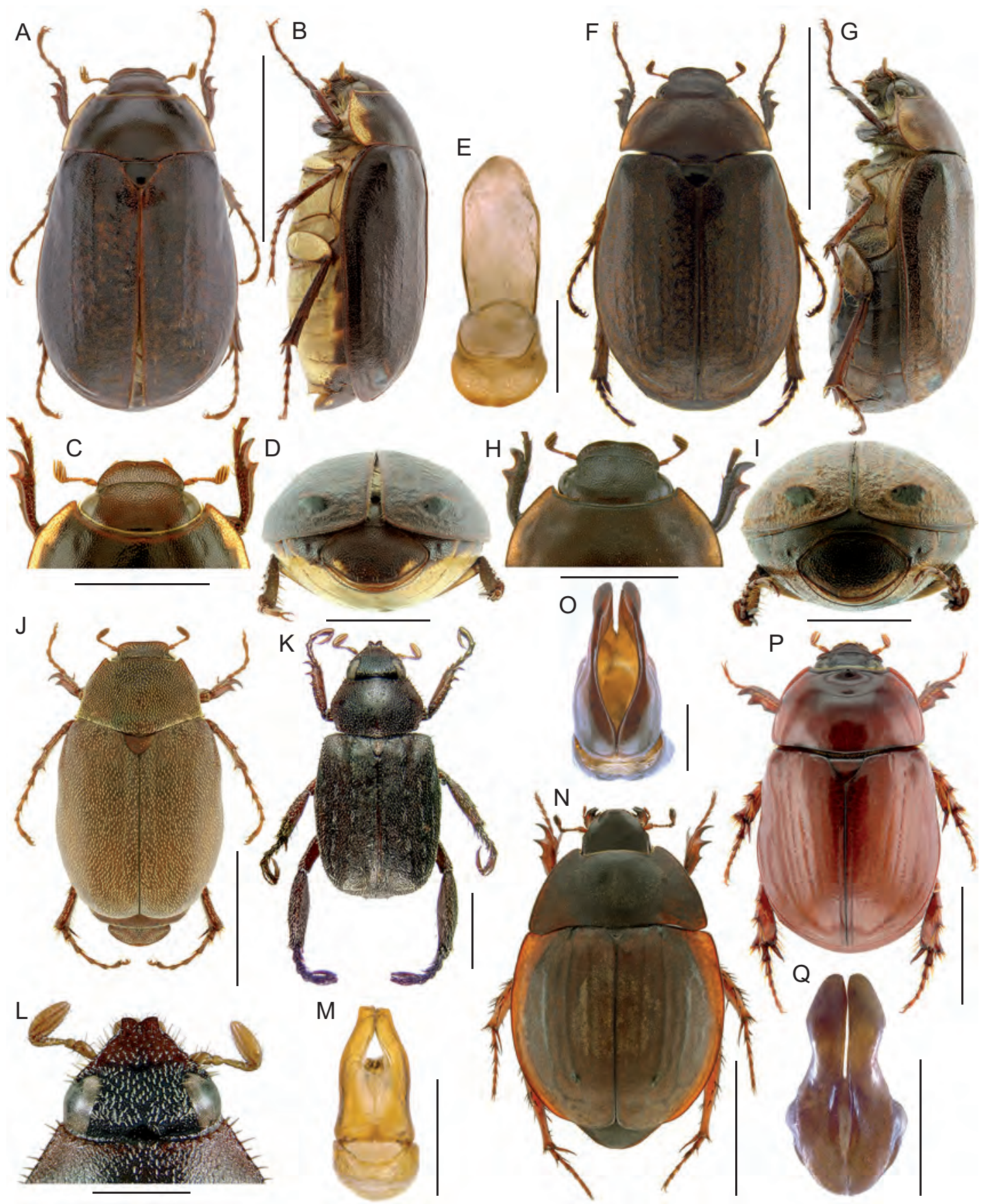

FIG. 24. - Imagos de Scarabaeoidea de Madagascar: A-E, Apicencya waterloti (Dewailly, 1950) mâle; A, habitus; B, face latérale; C, tête, face dorsale; D, habitus, vue apicale; E, édéage; F-I, Apicencya waterloti (Dewailly, 1950) femelle; F, habitus; G, face latérale; H, tête, face dorsale; I, habitus, vue apicale; J, Empecta scutata (Fairmaire, 1901), habitus; K-M, Paramorphochelus cornutus (Nonfried, 1892); K, habitus; L, tête, face dorsale; M, édéage; N, O, Hexodon unicolor (Olivier, 1789); N, habitus; O, édéage; $\mathbf{P}, \mathbf{Q}$, Paranodon coquerelii (Fairmaire, 1871); P, habitus; Q, édéage. Échelles: A, B, F, G, N, 10 mm; C, D, H-J, P, 5 mm; E, L, M, O, Q, 1 mm; K, 2 mm. 


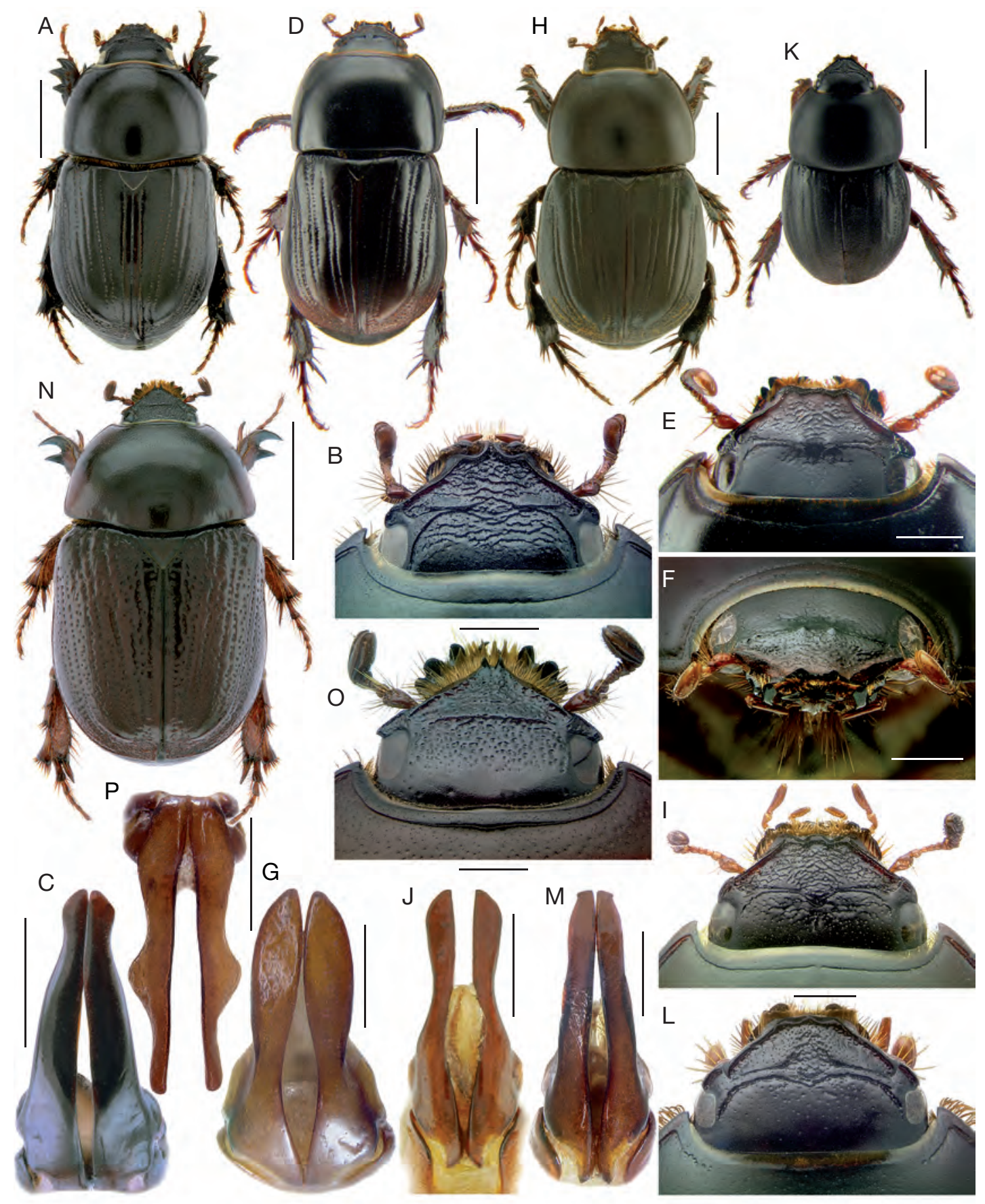

FIG. 25. - Imagos de Scarabaeoidea de Madagascar: A-C, Heteronychus arator rugifrons (Fairmaire, 1871); A, habitus; B, tête, face dorsale; C, édéage; D-G, Heteronychus bituberculatus (Kolbe, 1900); D, habitus; E, tête, face dorsale; F, tête vue de face; G, édéage; H-J, Heteronychus plebeius (Klug, 1833); H, habitus; I, tête, face dorsale; J, édéage; K-M, Heteronychus minutus (Burmeister, 1847); $\mathbf{K}$, habitus; L, tête, face dorsale; $\mathbf{M}$, édéage; N-P, Heteroconus paradoxus (Endrödi, 1968); N, habitus; O, tête, face dorsale; $\mathbf{P}$, édéage. Échelles: A, D, H, 3 mm; B, I, K, L, O, $1 \mathrm{~mm}$; C, E-G, J, M, P, 0,5 mm; N, 5 mm. 
pour les informations et les publications qu'il nous a transmises; M. J.-P. Lumaret, de l'Université Paul-Valéry à Montpellier, pour ses conseils et pour la documentation qu'il nous a procurée; M. M. Julien, directeur du CSIRO à Montpellier, pour la révision du texte anglais; Mme M. Duportal, pour la réalisation de la carte; $\mathrm{Mme} C$. Fovet Rabot, du Cirad, pour la participation au financement des figures en couleurs; Mme M. Fouillaud et MM. F. Chiroleu \& A. Rakotondrazaka et l'équipe de l'Unité de Recherches en Partenariat-Système de Culture et Riziculture durable (Scrid), plus particulièrement Mme J. Dusserre, MM. T. E Rajaonera \& E. Rafamatanantsoa, pour leur aide sur le terrain.

\section{RÉFÉRENCES}

ABERLENC H.-P. 2006. — Techniques entomologiques. L'entomologie sur Internet. L'Entomologiste 62 (1-2): 59-62.

APPERT J. 1968. - Les Insectes nuisibles aux cultures de Madagascar. IRAT, Bulletin agronomique 22, Antananarivo, $177 \mathrm{p}$.

BRENIÈRE J. \& DUbOIS J. 1965. - Catalogue des Insectes nuisibles aux cultures malgaches. Institut de Recherches agronomiques à Madagascar, Tananarive, Document 43, $168 \mathrm{p}$.

DeChambre R. P. 1986. — Insectes Coléoptères Dynastidae. Faune de Madagascar 65. Muséum national d'Histoire naturelle, Paris, 215 p.

Edmonds W. D. \& HalfFter G. 1978. - Taxonomic review of immature dung beetles of the subfamily Scarabaeinae (Coleoptera: Scarabaeidae). Systematic Entomology 3: 307-331

Jeanne I., Randremanana R., Robert V., Ariey F., TOMbo M. L., Wilmé L., RanivoARISOA S. \& DUCHEMIN J. B. 2002. - Biogéographie de Madagascar. In Atlas évolutif du paludisme à Madagascar. http:// www.pasteur.mg/IMG/pdf/Atlas-Palu.pdf (consulté en janvier 2010).

LACROIX M. 1989. - Insectes Coléoptères Melolonthidae. Faune de Madagascar 73 (1). Muséum national d'Histoire naturelle, Paris: 1-302.

LACroIX M. 1993. — Insectes Coléoptères Melolonthidae. Faune de Madagascar 73 (2). Muséum national d'Histoire naturelle, Paris: 303-875.

LACROIX M. 1997. — Insectes Coléoptères Hopliidae. Faune de Madagascar 88 (1), Muséum national d'Histoire naturelle, Paris: 1-399.
LACroIX M. 1998. — Insectes Coléoptères Hopliidae. Faune de Madagascar 88 (2), Muséum national d'Histoire naturelle, Paris: 400-755.

Paulian R. 1981. - Insectes Coléoptères Trogidae et Hybosoridae. Faune de Madagascar 56. Orstom \& CNRS, Paris: 1-28

Paulian R. 1984. - Les Orphnidae américains (Coléoptères, Scarabaeoidea). Annales de la Société entomologique de France (N.S.) 20 (1): 65-92.

Paulian R. \& Lumaret J.-P. 1982. - La larve des Orphnidae. Bulletin de la Société entomologique de France 87 (7-8): 263-272.

PeTERSON A. 1960. - Larvae of Insects. An Introduction to Nearctic Species. Part II. Coleoptera, Diptera, Neuroptera, Siphanoptera, Mecoptera, Trichoptera. Edwards Brothers Inc., Ann Arbor, Michigan, 416 p.

Peterson A. 1962. - Larvae of Insects. An Introduction to Nearctic Species. Part I. Lepidoptera and Plant Infesting Hymenoptera. Edwards Brothers Inc., Ann Arbor, Michigan, $315 \mathrm{p}$.

Randriamanantsoa R., Ratnadass A., AberlenC H.-P., RABEARISOA Y. M., RaJAONERA T. E., RAFAMATANANTSOA E. \& VERCAMBRE B. 2008. - Les vers blancs du riz pluvial d'altitude (Coleoptera Scarabaeoidea) à Madagascar: effets de la plante-hôte et de la matière organique du sol sur le comportement larvaire. Séminaire international «Les sols tropicaux en semis direct sous couvertures végétales", 3 au 8 décembre 2007, Antananarivo, Madagascar. Terre malgache 26: 35-37.

RATCliffe B. C. \& JAMESON M. L. 2004. - The revised classification for Scarabaeoidea: what the hell is going on? Papers in Entomology. University of Nebraska State: 3-10.

Ratnadass A., Michellon R., Randriamanantsoa R. \& SÉGUY L. 2006. - Effects of soil and plant management on crop pests and diseases, in UPHOFF N., Ball A., Fernandes E., Herren H., Husson O., Laing M., Palm C., Pretty J., Sanchez P., SANGINGA N. \& THIES J. (eds), Biological Approaches to Sustainable Soil Systems. CRC Press-Taylor \& Francis, Boca Raton, Florida: 589-602.

Ritcher P. O., 1966. - White Grubs and their Allies. A Study of North American Scarabaeoid Larvae. Oregon State Monographs, Studies in Entomology 4, Oregon State University Press, 219 p.

Vercambre B., Goebel O., Riba G., Morel G., Robert P. \& Guillon M. 1990. - Programme de lutte biologique contre Hoplochelus marginalis (Coleoptera: Melolonthinae), nouveau ravageur des cultures à l'île de la Réunion. Rencontres caraïbes en lutte biologique, Guadeloupe, 5-7 nov. 1990. Inra, Colloques 58: 371-378. 